\title{
INERTIAL MANIFOLDS FOR REACTION DIFFUSION EQUATIONS IN HIGHER SPACE DIMENSIONS
}

\author{
JOHN MALLET-PARET AND GEORGE R. SELL
}

\author{
1. Introduction \\ 2. An Abstract Invariant Manifold Theorem \\ 3. Verification of Conditions (I)-(IV) \\ 4. The Principle of Spatial Averaging \\ 5. The Uniform Cone Condition for $\Omega_{2}$ and $\Omega_{3}$ \\ Appendix A: Gap Theorems for Families of Quadratic Forms \\ References
}

\section{INTRODUCTION}

1.1. Inertial manifolds. It has been known for some time that many nonlinear dissipative evolutionary equations possess global attractors which have finite Hausdorff dimension, see Mallet-Paret (1976) and Mañé (1981). Some authors have studied specific systems with the goal of estimating the Hausdorff dimension, or the fractal dimension, in terms of the physical parameters of the problem. A partial listing of such results include Foias, and Temam (1979), Constantin and Foias (1985) and Constantin, Foias, and Temam (1985) for the Navier-Stokes equation, Foias, Manley and Temam (1986) for Benard convection and Nicolaenko, Scheurer and Temam $(1985,1987)$ for the KuramotoSivashinsky equation and the Cahn-Hilliard equation. See Hale (1988) and Temam (1988) for further references.

Because of this basic structure of the global attractor, it was widely believed that the long-time behavior of the solutions of a very large class of nonlinear evolutionary equations should strongly resemble the behavior of the solutions of a finite systems of ordinary differential equations. With the discovery recently that many of these equations (such as the Kuramoto-Sivashinsky equation) have

Received by the editors November 16, 1987.

1980 Mathematics Subject Classification (1985 Revision). Primary 35K57, 35P20, 34C30, 11B05, 11E25; Secondary 34C29, 47H10, 11N25.

Key words and phrases. Averaging, gaps, inertial manifolds, invariant manifolds, quadratic forms, reaction diffusion equations, spatial averaging.

Research on this project was done in part at the Division of Applied Mathematics at Brown University and the Institute for Mathematics and its Applications at the University of Minnesota and was supported in part by grants from the National Science Foundation and the Applied and Computational Mathematics Program at DARPA. 
inertial manifolds, one finds the beginnings of a theory which supports this conviction.

Since an inertial manifold is finite dimensional and invariant under the dynamics of the evolutionary equation, it follows that by restricting the evolutionary equation to this manifold one obtains a (finite) system of ODEs (ordinary differential equations). Since an inertial manifold is globally attracting (with asymptotic phase), it follows that every solution of the evolutionary equation converges to a solution of the ODE on the inertial manifold as time $t \rightarrow \infty$.

1.2. The spectral gap condition. An abstract form of the nonlinear evolutionary equation which arises in this theory is

$$
u^{\prime}+\nu A u=R(u)
$$

where $u^{\prime}=d u / d t, A$ is a selfadjoint operator with compact resolvent, $R(u)$ contains the nonlinearities and $\nu>0$. Let $\lambda_{1} \leq \lambda_{2} \leq \cdots \leq \lambda_{m} \rightarrow \infty$ denote the eigenvalues of $A$. With suitable assumptions on the nonlinearity $R(u)$, Foias, Sell, and Temam (1986) showed that there is a constant $c=c(\nu)$ such that if for some integer $N$ the gap condition:

$$
\lambda_{N+1}-\lambda_{N} \geq c\left(\lambda_{N}^{1 / 2}+\lambda_{N+1}^{1 / 2}\right)
$$

is satisfied, then equation (1.1) has an inertial manifold with dimension $N$. Since $c(\nu) \rightarrow \infty$ as $\nu \rightarrow 0$, it follows from (1.2) that $N \rightarrow \infty$ as $\nu \rightarrow 0$. This analysis applies in the case of the Kuramoto-Sivashinsky equation, see Foias, Nicolaenko, Sell, and Temam $(1985,1988)$.

Another theory of inertial manifolds appears in Mañé (1977), Henry (1981), Kamaev (1981), Mora (1983) as well as Foias, Sell and Temam (1986). In this case one assumes the nonlinearities $R(u)$ to be algebraic in $u$ and not involve fractional powers of $A$. For this problem a weaker gap condition,

$$
\lambda_{N+1}-\lambda_{N} \geq c,
$$

implies the existence of inertial manifolds. But even here one has $c(\nu) \rightarrow \infty$ as $\nu \rightarrow 0$. Therefore in order to insure the existence of inertial manifolds for every $\nu>0$ one uses

$$
\limsup _{m \rightarrow \infty}\left(\lambda_{m+1}-\lambda_{m}\right)=\infty .
$$

1.3. Reaction diffusion equations. The latter theory does show that the reaction diffusion equation

$$
u_{t}=\nu \Delta+f(x, u)
$$

with Dirichlet, Neumann, or periodic boundary conditions on $\Omega_{n}=(0,2 \pi)^{n} \mathrm{C}$ $R^{n}$ and $u \in R^{d}$ has an inertial manifold (under suitable assumptions on $f$ ) when $n=1,2$. If $n=1$, then $\lambda_{m} \sim \mathrm{cm}^{2}$ as $m \rightarrow \infty$ and the gap condition (1.4) follows from this fact. For $n=2$ one has $\lambda_{m} \sim \mathrm{cm}$, so that gap condition 
(1.4) is not immediate. However on the square $(0,2 \pi)^{2}$, the eigenvalues of $-\nu \triangle$ have the form

$$
\nu\left(k_{1}^{2}+k_{2}^{2}\right)
$$

where $k_{1}$ and $k_{2}$ are integers. In this case, a result from number theory implies that (1.4) is satisfied, cf. Richards (1982). More generally, if the square $\Omega_{2}$ is replaced by a rectangle $\left(0,2 \pi / a_{1}\right) \times\left(0,2 \pi / a_{2}\right)$ in $R^{2}$, then (1.4) is satisfied provided $\left(a_{1} / a_{2}\right)^{2}$ is rational. We do not know whether the gap condition (1.4) is satisfied if $\left(a_{1} / a_{2}\right)^{2}$ is irrational, nor whether (1.4) is satisfied for $-\nu \Delta$ on any other bounded two-dimensional domain.

1.4. Reaction diffusion equations in higher space dimension. Our investigation of inertial manifolds, which led to this paper, began with the simple question whether a spectral gap condition similar to (1.2), (1.3), or (1.4) was necessary for the existence of inertial manifolds. Since these gap conditions do not arise in the theories of Hausdorff dimension of global attractors, there seemed to be some hope in showing the existence of inertial manifolds without a spectral gap condition.

In order to address this question we began to investigate (1.5) on the region $\Omega_{n}=(0,2 \pi)^{n}$ for $n \geq 3$. In this setting one has (at least for Neumann or periodic boundary conditions)

$$
\begin{array}{ll}
\text { for } n=3: & \left(\lambda_{m+1}-\lambda_{m}\right)=0, \nu, 2 \nu, 3 \nu, \\
\text { for } n \geq 4: & \left(\lambda_{m+1}-\lambda_{m}\right)=0, \nu,
\end{array}
$$

cf. Hardy and Wright (1979, Chapter 20). In other words, the spectral gaps are small when $n \geq 3$. We further restricted our attention to the scalar equation (1.5), i.e., to $u \in R$. We shall assume that (1.5) is given with one of the following three boundary conditions:

$$
\begin{array}{ll}
\text { Dirichlet: } & u=0 \text { on } \partial \Omega_{n}, \\
\text { Neumann: } & \frac{\partial u}{\partial n}=0 \text { on } \partial \Omega_{n}, \\
\text { Periodic: } & u(t, x+2 \pi k)=u(t, x), \quad k \in Z^{n} .
\end{array}
$$

If the nonlinearity $f: \Omega_{n} \times R \rightarrow R$ is sufficiently smooth, say of class $C^{1}$, then for $t>0$ and for each $u_{0} \in L^{2}\left(\Omega_{n}\right)$ there is a solution $\dot{S}(t) u_{0}(x)=u(t, x)$ of (1.5) satisfying the given boundary conditions and such that $u(0, \cdot)=u_{0}(\cdot)$. Furthermore one has $S(t) u_{0} \in \mathscr{D}$, for $t>0$ where $\mathscr{D}$ denotes the domain of the associated selfadjoint operator $-\nu \Delta$ with the given boundary conditions, see Pazy (1983).

We are primarily interested in studying those equations (1.5) which are dissipative, even though some aspects of our theory do apply to nondissipative equations. By a dissipative equation we mean that the equation generates a nonlinear semiflow $S(t) u_{0}$ on some Hilbert space $H$ and that there is a bounded set 
$B \subset H$ such that for every $u_{0} \in H$ there is a time $T\left(u_{0}\right)$ such that $S(t) u_{0} \in B$ for all $t>T\left(u_{0}\right)$. For example, if for some $R>0$ the nonlinearity $f$ satisfies the sign condition:

$$
u f(x, u)<0 \quad \text { for all } x \in \Omega_{n} \text { and }|u| \geq R,
$$

then (1.5) is dissipative, Henry (1981).

When (1.5) is dissipative then there exists a global attractor $\mathfrak{A}$. This means that $\mathfrak{A}$ is a bounded set in $L^{\infty}$ and a compact set in $L^{2}$. Furthermore $\mathfrak{A}$ is invariant, it has finite Hausdorff dimension, and every solution $u(t, x)$ of $(1.5)$ satisfies

$$
u(t, x) \rightarrow \mathfrak{A} \quad \text { in } L^{\infty} \quad \text { as } t \rightarrow \infty .
$$

1.5. The main results. A set $\mathfrak{M} \subset L^{2}$ is said to be an inertial manifold for (1.5) if $\mathfrak{M}$ is a smooth $\left(C^{1}\right)$ finite dimensional manifold, $\mathfrak{A} \subset \mathfrak{M}$ and $\mathfrak{M}$ is invariant in the sense that if $u_{0} \in \mathfrak{M}$ then there is an $\varepsilon>0$ such that $S(t) u_{0} \in \mathfrak{M}$ for $0<t<\varepsilon$.

Our primary objective in this paper is to prove the following result:

Main Theorem. Assume that (1.5) is dissipative and that $f: \bar{\Omega}_{3} \times R \rightarrow R$ is of class $C^{3}$ on the cube $\Omega_{3}=(0,2 \pi)^{3}$. Then for every $\nu>0$ and for any choice of boundary conditions (Dirichlet, Neumann, or periodic), there exists an inertial manifold $\mathfrak{M}$ for (1.5).

Exactly the same result holds if the rectangular domain $\Omega_{2}=\left(0,2 \pi / a_{1}\right) \times$ $\left(0,2 \pi / a_{2}\right)$, where $a_{1}$ and $a_{2}$ are arbitrary positive numbers, is taken in place of the cube $\Omega_{3}$.

We emphasize that the result for $\Omega_{2}$ holds for any value of $\left(a_{1} / a_{2}\right)^{2}$, rational or irrational.

1.6. An outline of the proof. As in other theories for inertial manifolds, we find it convenient to modify the equation (1.5) outside of some neighborhood of the global attractor $\mathfrak{A}$. The modification we use is not standard. It is described in detail in $\S 3$.

Let $P$ denote the orthogonal projection of $L^{2}$ onto $\operatorname{Span}\left\{e_{1}, \ldots, e_{N}\right\}$, where $e_{1}, \ldots, e_{N}$ denotes the first $N$ eigenfunctions of $-\nu \triangle$, and let $Q=$ $I-P$. For $u \in L^{2}$ we define $p=P u$ and $q=Q u$. By applying $P$ and $Q$ to (1.5) we obtain the system

$$
\left\{\begin{array}{l}
p^{\prime}=\nu \Delta p+P \tilde{f}(p+q), \\
q^{\prime}=\nu \Delta q+Q \tilde{f}(p+q)
\end{array}\right.
$$

where $\tilde{f}(u)(x)=f(x, u(x))$. We are also interested in the induced linearized system

$$
\left\{\begin{array}{l}
\rho^{\prime}=\nu \triangle \rho+P D \tilde{f}(p+q)(\rho+\sigma), \\
\sigma^{\prime}=\nu \triangle \sigma+Q D \tilde{f}(p+q)(\rho+\sigma) .
\end{array}\right.
$$


Because we are assuming (1.5) to be a scalar problem ${ }^{1}$ (i.e., $u \in R$ ) and because the nonlinearity in (1.5) does not involve derivatives, the linear operator $D \tilde{f}$ appearing in (1.7) is in fact a multiplication operator

$$
(\rho+\sigma) \rightarrow v(\rho+\sigma)
$$

where $v(x)=D_{u} f(x, u(x))$. This simple observation introduces a significant simplification into the study of (1.7) and (1.6).

Our principal objective is to show that, for an appropriate choice of the dimension $N$, equation (1.7) satisfies a cone condition (i.e., has an exponential dichotomy) over a suitable portion of the space $L^{2}$. This cone condition is the main hypothesis in the (Abstract) Invariant Manifold Theorem proved in $\S 2$. The remainder of the paper consists of showing that this Invariant Manifold Theorem does apply to the modified form of (1.5).

The techniques used to prove the Invariant Manifold Theorem in $\S 2$ are adaptations of the classical graph transform method due to Hadamard. This method is a standard technique used in finite dimensional dynamical systems to construct locally invariant manifolds, such as the center manifold and the center-unstable manifold. See, for example, Hartman (1964) and Hirsch, Pugh, and Shub (1977). While our theory is based on the graph transform method, there are a number of issues which arise in the infinite dimensional setting which are minor matters in the finite dimensional problem. Many of these issues are technical in nature and will be described further in the body of the paper. Because of our emphasis on inertial manifolds, we seek conditions on the abstract evolutionary equation which will guarantee the existence of a global (as opposed to local) invariant manifold.

Cone conditions similar to ours have been used to prove the existence of globally invariant finite dimensional manifolds for infinite dimensional dynamical systems. See, for example, Constantin, Foias, Nicolaenko, and Temam (1986, $1988,1989)$ and Foias, Sell, and Titi (1988). We shall show in $\S 4$ that the spectral gap condition (1.4) implies the cone condition, in the setting of reaction diffusion equations. A similar argument is used in many of the applications of the cone conditions in Constantin, Foias, Nicolaenko, and Temam (1986, 1988 , 1989), i.e., a spectral gap condition implies a cone condition. The main contribution of the present paper is to derive an alternate theory for inertial manifolds, a theory which does not rely on a spectral gap condition.

An alternate method for proving the existence of inertial manifolds is the Lyapunov-Perron method, which is based on the variation of constants formula. Many of the existing theories of inertial manifolds use this method. See, for example, Henry (1981), Kamaev (1981), Mora (1983), Foias, Sell, and Temam (1986), Nicolaenko, Scheurer, and Temam (1987), Foias, Nicolaenko, Sell, and Temam (1988), and Mora and Solà-Morales (1988). Our decision to use the graph transform method in place of the Lyapunov-Perron method in this paper

\footnotetext{
${ }^{1}$ Another reason for restricting to scalar equations is given in Mallet-Paret and Sell (1988).
} 
was not a matter of choice. Instead, we wished to exploit an advantage which occurs when one uses the Hadamard approach in connection with the multiplication operator described in $\S 1.4$. This advantage is an algebraic cancellation, ${ }^{2}$ which occurs in the verification of the cone condition.

1.7. The Principle of Spatial Averaging. We shall rewrite (1.7) as

$$
\left\{\begin{array}{l}
\rho^{\prime}=\nu \Delta \rho+P v(\rho+\sigma), \\
\sigma^{\prime}=\nu \Delta \sigma+Q v(\rho+\sigma) .
\end{array}\right.
$$

The key idea in showing that (1.8) has an exponential dichotomy (i.e. satisfies the cone condition) is that for certain regions $\Omega \subset R^{n}, n \leq 3$, equation (1.8) can be "well approximated" by the constant coefficient equation

$$
\left\{\begin{array}{l}
\rho^{\prime}=\nu \Delta \rho+P \bar{v}(\rho+\sigma)=\nu \Delta \rho+\bar{v} \rho, \\
\sigma^{\prime}=\nu \Delta \sigma+Q \bar{v}(\rho+\sigma)=\nu \Delta \sigma+\bar{v} \sigma
\end{array}\right.
$$

where $\bar{v}=(\operatorname{vol} \Omega)^{-1} \int_{\Omega} v d x$. Notice that the term $\bar{v}$ in (1.9) introduces a uniform shift in the spectrum of (1.9)

$$
\nu \lambda_{m} \rightarrow \nu \lambda_{m}-\bar{v} \quad \text { for all } m \text {. }
$$

If $O(\xi)$ measures the error with which (1.9) approximates (1.8) and if for some $N$ the eigenvalues of $-\nu \triangle$ satisfy $\nu\left(\lambda_{N+1}-\lambda_{N}\right)>2 O(\xi)$ then for the eigenvalues of (1.8) one has

$$
\nu \lambda_{N+1}-\bar{v}-O(\xi)>\nu \lambda_{N}-\bar{v}+O(\xi),
$$

which implies the cone condition.

The mathematical basis for justifying the approximations described in the last paragraph is the Principle of Spatial Averaging, which we introduce in $\S 4$. The Principle of Spatial Averaging is a property which the Laplacian over a bounded Lipschitz region $\Omega \subset R^{n}, n \leq 3$, may (or may not) have. What we show is that the conclusions of the Main Theorem hold over any region $\Omega$ for which the Principle of Spatial Averaging holds.

1.8. A number theoretic theorem. It remains to show that the Laplacian satisfies the Principle of Spatial Averaging over the regions $\Omega_{2}$ and $\Omega_{3}$ described in the Main Theorem. This argument is presented in $\S 5$ and Appendix A.

As we show, the argument reduces to proving a Gap Theorem for a finite collection of quadratic forms with rational coefficients. This Gap Theorem, which is an extension of a result of Richards (1982), is proved in Appendix A. An example of the Gap Theorem is that the union of the following three sets has arbitrarily large gaps in $Z_{+}=\{1,2,3, \ldots\}$ :

$$
\begin{gathered}
\left\{m^{2}+n^{2}: m, n \in Z\right\}, \quad\left\{m^{2}+7 n^{2}: m, n \in Z\right\} \\
\left\{3 m^{2}-4 m n+3 n^{2}+5 m+11 n: m, n \in Z\right\} .
\end{gathered}
$$

${ }^{2}$ See equation (4.8). 
Remarks. While we shall not present the details here, it should be noted that the methods of this paper readily extend the Main Theorem to study of (1.5) on the parallelepiped

$$
\Omega_{3}=\left(0,2 \pi / a_{1}\right) \times\left(0,2 \pi / a_{2}\right) \times\left(0,2 \pi / a_{3}\right)
$$

provided all the squares of ratios $\left(a_{i} / a_{j}\right)^{2}$ are rational. ${ }^{3}$

We are grateful to Ian Richards for his helpful comments on the numbertheoretic aspects of this paper.

\section{AN ABSTRACT INVARIANT MANIFOLD THEOREM}

2.1. Nonlinear dynamics and the variational equation. Let $\mathscr{H}$ be a Hilbert space with inner product $\langle\cdot, \cdot\rangle$ and norm $\|\cdot\|$, let $\mathscr{P}$ be a finite dimensional subspace of $\mathscr{H}$ with orthogonal projection $P$, and let $\mathscr{Q}=\mathscr{P}^{\perp}$ be the orthogonal complement with complementary projection $Q=I-P$. Writing $u \in \mathscr{H}$ as $u=(p, q)$ where

$$
p=P u \in \mathscr{P}, \quad q=Q u \in \mathscr{Q},
$$

we consider an abstract differential equation

$$
\begin{aligned}
& p^{\prime}=F(p, q) \\
& q^{\prime}=-A q+G(p, q) .
\end{aligned}
$$

(We emphasize the notational difference between the inner product $\langle\cdot, \cdot\rangle$ on $\mathscr{H}$ and the Cartesian product $(\cdot, \cdot)$ with $\mathscr{H}=\mathscr{P} \times \mathscr{Q}$.) We assume $A$ is a closed, selfadjoint linear operator on $\mathscr{Q}$ with dense domain $\mathscr{D} \subset \mathscr{Q}$. We assume further that $-A$ generates a $C^{0}$-semigroup $e^{-A t}$ in $\mathscr{Q}$ for $t>0$, and also that $A$ has a compact resolvent on $\mathscr{Q}$. The (nonlinear) functions

$$
F: \mathscr{H} \rightarrow \mathscr{P}, \quad G: \mathscr{H} \rightarrow \mathscr{Q}
$$

are assumed to be locally Lipschitz continuous in $\mathscr{H}$. These conditions on $A, F$, and $G$ are standing assumptions, to hold for the remainder of this section. We do not write the finite dimensional equation for $p$ with a separate linear part, instead incorporating this into the function $F$.

With initial conditions $\left(p_{0}, q_{0}\right) \in \mathscr{P} \times \mathscr{Q}$ we have the associate integral equations

$p(t)=p_{0}+\int_{0}^{t} F(p(s), q(s)) d s, \quad q(t)=e^{-A t} q_{0}+\int_{0}^{t} e^{-A(t-s)} G(p(s), q(s)) d s$.

A mild solution $u(t)=(p(t), q(t))$ of $(2.1)$ is defined to be a solution of this integral equation on some interval $0<t<a$. It is known ${ }^{4}$ that under the assumptions given above, for every $\left(p_{0}, q_{0}\right) \in \mathscr{P} \times \mathscr{Q}$ there is a unique mild solution $u(t)=(p(t), q(t))$ of $(2.1)$ on some maximal interval $[0, \omega)$ for

\footnotetext{
${ }^{3}$ Compare this with the situation described in $\S 1.3$.

${ }^{4}$ Pazy (1983) is a good reference for the semigroup theory we outline in this section. Also see Henry (1981).
} 
some $\omega=\omega\left(p_{0}, q_{0}\right) \in(0, \infty]$. This solution is continuous for $t \in[0, \omega)$. Furthermore if $q_{0} \in \mathscr{D}$ then this solution is Lipschitz continuous in $t$ and it is a strong solution of $(2.1)$, which means that the following two conditions hold almost everywhere on $(0, \omega)$ :

(1) the time derivative $u^{\prime}(t)$ exists and $u(t)$ is a solution of (2.1),

(2) $u(t) \in \mathscr{D}$.

If $\omega<\infty$ then this solution eventually leaves each compact set $K \subset \mathscr{H}$ as $t \rightarrow \omega^{-}$, i.e., one has $u(t) \notin K$ for all $t \in(\omega-\varepsilon, \omega)$, for some $\varepsilon=$ $\varepsilon\left(p_{0}, q_{0}, K\right)>0$. Denoting the solution $u(t)$ by

$$
u\left(t, u_{0}\right)=\left(p\left(t, p_{0}, q_{0}\right), q\left(t, p_{0}, q_{0}\right)\right)
$$

where $u_{0}=\left(p_{0}, q_{0}\right)$, one can further prove, in a standard fashion, that the set

$$
\mathscr{O}=\left\{\left(t, u_{0}\right): t \in\left[0, \omega\left(u_{0}\right)\right) \text { and } u_{0} \in \mathscr{H}\right\}
$$

is open in $[0, \infty) \times \mathscr{H}$, and that the solution function $u\left(t, u_{0}\right)$ is continuous in each variable in $\mathscr{O}$.

As an aside we recall that if $-A$ generates an analytic semigroup, as is the case when $A$ is selfadjoint with compact resolvent, then the solution $u(t)$ is a classical solution of $(2.1)$ for all $t>0$, i.e., $u(t)$ is a $C^{1}$ function in $t$ and satisfies $(2.1)$ everywhere on $(0, \omega)$.

If $F$ and $G$ are $C^{1}$ functions, then standard regularity results, relating smooth dependence on initial conditions to solutions of the linear variational equation

$$
\begin{aligned}
& \rho^{\prime}=D F(p, q)(\rho, \sigma), \\
& \sigma^{\prime}=-A s+D G(p, q)(\rho, \sigma)
\end{aligned}
$$

along a solution $(p, q)=(p(t), q(t))$, would hold. However, for technical but essential reasons, $F$ and $G$ will not be assumed $C^{1}$; rather they will satisfy a weaker smoothness condition. As we will see shortly, enough regularity will be present so that the variational equation (2.2) will make sense for certain solutions $(p(t), q(t))$ of $(2.1)$.

Under appropriate assumptions, we shall prove the existence of a $C^{1}$ invariant manifold $\mathfrak{M}$ for (2.1). In fact, $\mathfrak{M}$ will be the graph

$$
\mathfrak{M}=\{(p, \Phi(p)): p \in \mathscr{P}\}
$$

of a $C^{1}$ function $\Phi: \mathscr{P} \rightarrow \mathscr{Q}$. In particular, $\mathfrak{M}$ will have finite dimension

$$
\operatorname{dim} \mathfrak{M}=\operatorname{dim} \mathscr{P}<\infty .
$$

Before presenting the precise conditions needed to obtain $\mathfrak{M}$, we make a definition which generalizes the notion of a Fréchet derivative. We let $\|\cdot\|_{X}$ denote the norm in a Banach space $\mathscr{X}$. We also let $\mathscr{L}(\mathscr{X}, \mathscr{Y})$ denote the Banach space of bounded linear transformations from $\mathscr{X}$ to $\mathscr{Y}$, where $\mathscr{Y}$ is another Banach space. 
Definition. Let $\mathscr{X}$ and $\mathscr{Y}$ be Banach spaces, and $\mathscr{K} \subset \mathscr{X}$ a closed convex set. A function $H: \mathscr{K} \rightarrow \mathscr{Y}$ is said to be $C^{1}$ in $\mathscr{K}$ if for each $x \in \mathscr{K}$ there exists a bounded linear transformation $L=L(x) \in \mathscr{L}(\mathscr{X}, \mathscr{Y})$ such that $L(x)$ varies continuously as an element of $\mathscr{L}(\mathscr{X}, \mathscr{Y})$ for $x \in \mathscr{K}$, and for each $\varepsilon>0$ there exists $\delta=\delta(x, \varepsilon)>0$ such that

$$
\|H(x+h)-H(x)-L h\|_{Y} \leq \varepsilon\|h\|_{X}
$$

whenever $0<\|h\|_{X}<\delta$, and both $x, x+h \in \mathscr{K}$. For $x \in \mathscr{K}$ we write $D H(x)=L(x)$ and call this operator the derivative of $H$ at $x$.

The derivative $L(x)$ is uniquely determined for each $x \in \mathscr{K}$ as long as $\mathscr{K}$ is not a subset of a proper closed affine subspace of $\mathscr{X}$. It is this situation which arises in our applications.

Observe that if $x$ is an interior point of $\mathscr{K}$, then $D H(x)$ is simply the Fréchet derivative of $H$; but in general it need not be a Fréchet derivative. (For example, the interior of $\mathscr{K}$ may be empty.) Also note that along the line segment joining any two points $x_{1}, x_{2} \in \mathscr{K}$ the function

$$
\tilde{H}(\theta)=H\left(x_{1}+\theta\left(x_{2}-x_{1}\right)\right)
$$

is $C^{1}$ in the usual sense, for $\theta \in(0,1)$. One has, therefore, the Mean Value Formula

$$
\begin{aligned}
H\left(x_{2}\right)-H\left(x_{1}\right) & =\int_{0}^{1} \frac{d}{d \theta} \tilde{H}(\theta) d \theta \\
& =\left[\int_{0}^{1} D H\left(x_{1}+\theta\left(x_{2}-x_{1}\right)\right) d \theta\right]\left(x_{2}-x_{1}\right) .
\end{aligned}
$$

For the functions of interest the set $\mathscr{K}$ will have empty interior, but will be convex so the above formula is applicable.

2.2. The main hypotheses. With the above standing assumptions on $A, F$, and $G$, the existence of the invariant manifold $\mathfrak{M}$ for the equation (2.1) will be proved under the following five hypotheses. The last of these hypotheses, the Cone Condition, plays an especially important role in the theory we present below.

\section{The main hypotheses.}

I. (Regularity Condition) There exist constants $R_{1}$ and $R_{2}$ such that both $F$ and $G$ are $C^{1}$ in the convex set $\mathscr{A} \times \mathscr{C}$, where ${ }^{5}$

$$
\begin{aligned}
& \mathscr{A}=\left\{p \in \mathscr{P}:\|p\| \leq R_{1}\right\}, \\
& \mathscr{C}=\left\{q \in \mathscr{D} \subset \mathscr{Q}:\|A q\| \leq R_{2}\right\} .
\end{aligned}
$$

II. (Dissipative Condition) If $p \in \operatorname{cl}(\mathscr{P} \backslash \mathscr{A})$ then $\langle p, F(p, 0)\rangle<0$ and $G(p, 0)=0 .^{6}$

\footnotetext{
${ }^{5}$ Note that, since $A$ has compact resolvent, the set $\mathscr{C}$ is compact in $\mathscr{Q}$.

${ }^{6} \mathrm{We}$ use $\mathrm{cl}$ to denote the closure operation.
} 


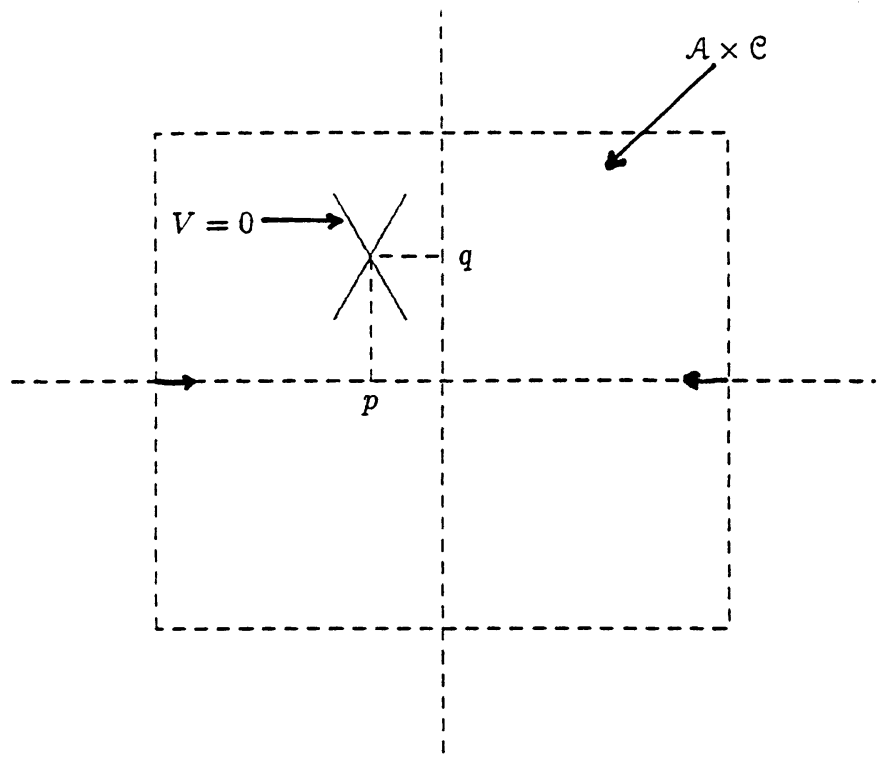

Figure 1. The region $\mathscr{A} \times \mathscr{C}$ in $\mathscr{P} \times \mathscr{Q}$.

III. (Sobolev Condition) If $p_{0} \in \mathscr{A}$ and $t_{0}>0$ are such that $p\left(t, p_{0}, 0\right) \in$ $\mathscr{A}$ holds in $\left[0, t_{0}\right]$, then one also has $q\left(t, p_{0}, 0\right) \in \mathscr{C}$ in $\left[0, t_{0}\right]$.

IV. (Linear Stability Condition) One has $\langle q, A q\rangle \geq \Lambda\|q\|^{2}$ for all $q \in \mathscr{D}$, for some $\Lambda>2 \gamma$, where $\gamma=\sup \left\{\|D G(p, q)\|_{L}:(p, q) \in \mathscr{A} \times \mathscr{C}\right\}$, and $L=\mathscr{L}(\mathscr{H}, \mathscr{Q})$.

V. (Cone Condition) With $V \stackrel{\text { def }}{=} \frac{1}{2}\|\sigma\|^{2}-\frac{1}{2}\|\rho\|^{2}$ and $V^{\prime} \stackrel{\text { def }}{=}\left\langle\sigma, \sigma^{\prime}\right\rangle-\left\langle\rho, \rho^{\prime}\right\rangle$, where $\rho \in \mathscr{P}, \sigma \in \mathscr{D} \subset \mathscr{Q}$, and $\rho^{\prime}$ and $\sigma^{\prime}$ are given by the variational equation (2.2), one has $V^{\prime}<0$ whenever $(p, q) \in \mathscr{A} \times \mathscr{C}$ and $\|\rho\|=$ $\|\sigma\| \neq 0$.

One may obtain a better understanding of the analytical and geometrical significance of these five conditions with the following observations: The Regularity Condition arises naturally when studying nonlinear reaction diffusion equations of the type described in the Introduction. For example, the mapping $f(u)(t)=\sin u(t)$ is $C^{1}$ (even analytic) on $H^{1}(0,1)$ but nowhere differentiable on $L^{2}(0,1)$, cf. Henry $(1981)$. The Dissipative Condition means that the vector field described in (2.1) is pointing inward on the boundary of $\mathscr{A} \subset \mathscr{P}$, see Figure 1. We use the term Sobolev Condition for condition (III) because the verification of this involves the derivation of a priori bounds by means of various Sobolev inequalities, see $\S 3.6$. The Sobolev Condition asserts that solutions $(p(t), q(t))$ of $(2.1)$, with initial conditions on the $\mathscr{P}_{\text {-axis, remain in }}$ $\mathscr{A} \times \mathscr{C}$ provided $\|p(t)\| \leq R_{1}$. In other words, these solutions $(p(t), q(t))$ cannot leave $\mathscr{A} \times \mathscr{C}$ through the "top." The Linear Stability Condition, especially the inequality $\Lambda>2 \gamma$, asserts that the attraction of the linear part of 
the $q$-equation dominates the distortion of the nonlinear terms. If the $p$-terms were missing, this condition is a statement about the asymptotic stability of the $q$-equation.

As mentioned above, the Cone Condition plays an especially important role in our theory. What it says is that the cone "bundle"

$$
\mathfrak{V}=\{(p, q ; \rho, \sigma):(p, q) \in \mathscr{A} \times \mathscr{C} \text { and }\|\rho\| \leq\|\sigma\|\}
$$

is positively invariant for the variational equation (2.2).

Let $u_{j}(t)=\left(p_{j}(t), q_{j}(t)\right)$ for $j=1,2$, be two solutions of (2.1) which for some range of $t$ belong to $\mathscr{A} \times \mathscr{C}$. By subtracting the differential equations for these solutions and using the mean value formula (2.3) one obtains

$$
\begin{aligned}
& \rho^{\prime}=\left[\int_{0}^{1} D F\left(p_{1}+\theta\left(p_{2}-p_{1}\right), q_{1}+\theta\left(q_{2}-q_{1}\right)\right) d \theta\right](\rho, \sigma), \\
& \sigma^{\prime}=-A \sigma+\left[\int_{0}^{1} D G\left(p_{1}+\theta\left(p_{2}-p_{1}\right), q_{1}+\theta\left(q_{2}-q_{1}\right)\right) d \theta\right](\rho, \sigma),
\end{aligned}
$$

where $p_{j}=p_{j}(t), q_{j}=q_{j}(t)$, and $\rho$ and $\sigma$ are the differences

$$
\rho(t)=p_{2}(t)-p_{1}(t), \quad \sigma(t)=q_{2}(t)-q_{1}(t) .
$$

If for some $t$ one has $\|\rho(t)\|=\|\sigma(t)\| \neq 0$, then by applying the Cone Condition (V) to the integrand of $(2.4)$ for each $\theta \in[0,1]$ and computing the time derivative of $W$ with respect to (2.4), $W^{\prime}=\left\langle\sigma, \sigma^{\prime}\right\rangle-\left\langle\rho, \rho^{\prime}\right\rangle$, one obtains the inequality

$$
\begin{aligned}
W^{\prime}=-\langle\sigma, A \sigma\rangle & +\int_{0}^{1}\left\langle\sigma, D G\left(p_{1}+\theta\left(p_{2}-p_{1}\right), q_{1}+\theta\left(q_{2}-q_{1}\right)\right)(\rho, \sigma)\right\rangle d \theta \\
& \quad-\int_{0}^{1}\left\langle\rho, D F\left(p_{1}+\theta\left(p_{2}-p_{1}\right), q_{1}+\theta\left(q_{2}-q_{1}\right)\right)(\rho, \sigma)\right\rangle d \theta \\
& <0 .
\end{aligned}
$$

Note also that in verifying the Cone Condition one may assume without loss of generality that $\|\rho\|=\|\sigma\|=1$, as $V^{\prime}$ is homogeneous in $(\rho, \sigma)$.

2.3. The Invariant Manifold Theorem. The following Invariant Manifold Theorem for the abstract system (2.1) is the main result of this section. The proof of this theorem follows from a sequence of lemmas and propositions which constitutes the bulk of this section.

Before stating the theorem we introduce the following notation: Let $\Phi: \mathscr{P} \rightarrow$ $\mathscr{Q}$ be a function. The graph and the support of $\Phi$ are

$$
\begin{gathered}
\operatorname{graph}(\Phi)=\{(p, \Phi(p)): p \in \mathscr{P}\}, \\
\operatorname{supp} \Phi=\operatorname{cl}\{p \in \mathscr{P}: \Phi(p) \neq 0\} .
\end{gathered}
$$

Also we will let $\mathscr{E}$ denote the following subset of $\mathscr{A} \times \mathscr{C}$ :

$$
\mathscr{E}=\{(p, q) \in \mathscr{A} \times \mathscr{C}:\|q\| \leq \operatorname{dist}(p, \text { bdy } \mathscr{A})\} .
$$




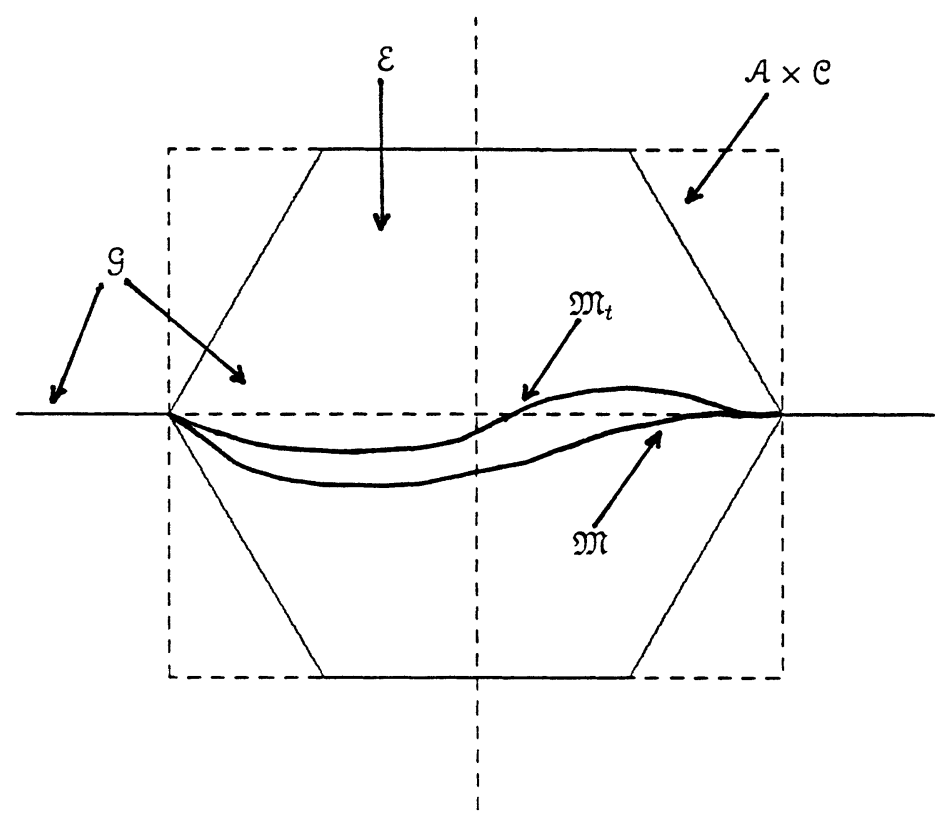

Figure 2. The subsets $\mathscr{E}$ and $\mathscr{G}$. The manifolds $\mathfrak{M}_{t}$ and $\mathfrak{M}$.

Finally we let

$$
\mathscr{G}=\mathscr{E} \cup(\mathscr{P} \times\{0\})
$$

see Figure 2.

Theorem A. Assume the differential equation (2.1) satisfies conditions (I)-(V) in addition to the standing assumptions on $A, F$, and $G$ given above. Then there exists a Lipschitz function $\Phi: \mathscr{P} \rightarrow \mathscr{Q}$ with Lipschitz constant at most one, satisfying

$$
\begin{gathered}
\Phi(p) \in \mathscr{C} \quad \text { for } p \in \mathscr{P}, \\
\operatorname{supp} \Phi \subset \mathscr{A}
\end{gathered}
$$

and such that the graph, $\mathfrak{M}=\operatorname{graph}(\Phi)$, is an invariant manifold for (2.1) with $\mathfrak{M} \subset \mathscr{G}$, where $\mathscr{G}$ is given by (2.6). Furthermore $\mathfrak{M}$ is locally attracting in the following sense: there exists an $\alpha>0$ such that if $u(t)=(p(t), q(t))$ is $a$ solution of (2.1) satisfying $u(t) \in \mathscr{E}$ for all $t>0$, then

$$
\operatorname{dist}(u(t), \mathfrak{M}) \leq 2 e^{-\alpha t}(\operatorname{diam} \mathscr{C}), \quad t>0
$$

That is, $u(t)$ approaches $\mathfrak{M}$ at a uniform exponential rate.

It is thus the case that any solution $p(t)$ of the ordinary differential equation

$$
p^{\prime}=F(p, \Phi(p))
$$


yields a solution $(p(t), q(t))=(p(t), \Phi(p(t)))$ of $(2.1)$. We also note that if $(p(t), q(t))$ is a solution of $(2.1)$ defined for all $t \in(-\infty, \infty)$ and satisfying $(p(t), q(t)) \in \mathscr{A} \times \mathscr{C}$ for all $t$, then in fact one has

$$
(p(t), q(t)) \in \mathfrak{M}, \quad \text { for all } t
$$

thus $p(t)$ satisfies (2.7) and $q(t)=\Phi(p(t))$. In particular, in the applications of Theorem A, it will follow that the global attractor $\mathfrak{A}$ of $(2.1)$ must lie in $\mathfrak{M}$ and the flow on $\mathfrak{A}$ is given by (2.7).

2.4. Outline of the proof of Theorem A. We begin the proof of Theorem A by first noting that without loss of generality we may assume

$$
F(p, 0)=-p \quad \text { for } p \in \mathscr{P} \backslash 2 \mathscr{A},
$$

where $2 \mathscr{A}=\{2 p: p \in \mathscr{A}\}$ is the ball in $\mathscr{P}$ with twice the radius of $\mathscr{A}$. Indeed if (2.8) is not satisfied then we can replace $F$ with the new nonlinearity:

$$
\tilde{F}(p, q)=\zeta(p) F(p, q)-(1-\zeta(p)) p,
$$

where $\zeta: \mathscr{P} \rightarrow[0,1]$ is a smooth function satisfying

$$
\begin{array}{ll}
\zeta(p)=1 & \text { for } p \in \mathscr{A}, \\
\zeta(p)=0 & \text { for } p \in \mathscr{P} \backslash 2 \mathscr{A},
\end{array}
$$

and observe that conditions $(\mathrm{I})-(\mathrm{V})$ still hold for $\widetilde{F}$. One reason for so modifying $F$ is that each solution $p(t)$ of the ordinary differential equation $p^{\prime}=$ $\widetilde{F}(p, 0)$ exists for all $t \in(-\infty, \infty)$. To conserve notation we shall denote $\widetilde{F}$ simply by $F$, assuming from now on that (2.8) holds.

Our strategy for constructing the invariant manifold $\mathfrak{M}$ is the following: We begin with finite dimensional set $\mathscr{P} \times\{0\}$, which we denote by $\mathfrak{M}_{0}$. Next we let the dynamics of (2.1) act on $\mathfrak{M}_{0}$ for $t>0$. This yields the set $\mathfrak{M}_{t} \subset \mathscr{H}$, which is defined to be the image of $\mathfrak{M}_{0}$ under the flow at time $t$. We shall show that for each $t>0$ there is a Lipschitz function $\Phi_{t}: \mathscr{P} \rightarrow \mathscr{Q}$ such that $\mathfrak{M}_{t}=$ graph $\Phi_{t}$. In addition, we will show that for each $t$, the function $\Phi_{t}$ has a Lipschitz constant at most one, and the limit $\Phi_{t} \rightarrow \Phi$ as $t \rightarrow \infty$ will exist with a uniform exponential rate. The desired invariant manifold $\mathfrak{M}$ will be given as the graph of this limiting function:

$$
\mathfrak{M}=\operatorname{graph} \Phi \text {. }
$$

A regularity result will in fact insure that $\mathfrak{M}$ is $C^{1}$ and exponentially stable.

2.5. Properties of $\mathfrak{M}_{t}$. Let us therefore define the sets $\mathfrak{M}_{t} \subset \mathscr{H}$ by

$$
\mathfrak{M}_{t}=\left\{u\left(t, p_{0}, 0\right): p_{0} \in \mathscr{P} \text { and } t \in\left[0, \omega\left(p_{0}, 0\right)\right)\right\} .
$$

The next few results describe some basic properties of $\mathfrak{M}_{t}$. 
Lemma 2.1. If $p_{0} \in \mathscr{A}$ then $u\left(t, p_{0}, 0\right)$ exists for all $t>0$ and satisfies

$$
u\left(t, p_{0}, 0\right) \in \mathscr{A} \times \mathscr{C}
$$

for all $t>0$. Furthermore, one has

$$
\left\|q\left(t, p_{0}, 0\right)\right\|<\operatorname{dist}\left(p\left(t, p_{0}, 0\right), \operatorname{bdy} \mathscr{A}\right)
$$

for all $t>0$. In particular one has $u\left(t, p_{0}, 0\right) \in \mathscr{E}$ for all $t>0$.

Proof. If $p_{0} \in$ int $\mathscr{A}$ then the Sobolev Condition (III) implies that (2.9) holds for all small $t>0$; also, (2.10) holds for small $t>0$ by continuity.

Now suppose $p_{0} \in$ bdy $\mathscr{A}$. We claim that again both (2.9) and (2.10) hold for small $t>0$. For (2.9) we first observe that by the Dissipative Condition (II) one has

$$
\left.\frac{d}{d t}\left\|p\left(t, p_{0}, 0\right)\right\|^{2}\right|_{t=0}=2\left\langle p_{0}, F\left(p_{0}, 0\right)\right\rangle<0,
$$

hence

$$
\left.\frac{d}{d t}\left[\operatorname{dist}\left(p\left(t, p_{0}, 0\right), \text { bdy } \mathscr{A}\right)\right]\right|_{t=0}>0
$$

and $p\left(t, p_{0}, 0\right) \in$ int $\mathscr{A}$ for small $t>0$. The validity of (2.9) for small $t>0$ follows from the Sobolev Condition (III). Since

$$
\left.\frac{d}{d t} q\left(t, p_{0}, 0\right)\right|_{t=0}=G\left(p_{0}, 0\right)=0
$$

and since both sides of (2.10) equal zero when $t=0$, one concludes that (2.10) is also valid for small $t>0$.

Now let $\left(0, t_{0}\right)$ denote the maximal interval in which (2.10) holds. For all $t \in$ $\left(0, t_{0}\right)$ one has $p\left(t, p_{0}, 0\right) \in$ int $\mathscr{A}$, since $p\left(t, p_{0}, 0\right) \notin$ bdy $\mathscr{A}$. Consequently (2.9) is valid for $t \in\left(0, t_{0}\right)$ by the Sobolev Condition (III). Therefore it is sufficient to show that $t_{0}=\infty$ to prove the lemma.

Suppose on the contrary that $t_{0}<\infty$. The compactness of $\mathscr{A} \times \mathscr{C}$ and the fact that the solution $u\left(t, p_{0}, 0\right)$ remains in $\mathscr{A} \times \mathscr{C}$ throughout $\left[0, t_{0}\right)$ implies that this interval is not the maximal interval of existence. In particular, $u\left(t, p_{0}, 0\right)$ is defined at $t=t_{0}$. By the definition of $t_{0}$, the relation (2.10) becomes an equality at $t=t_{0}$, i.e.,

$$
\left\|q\left(t_{0}, p_{0}, 0\right)\right\|=\operatorname{dist}\left(p\left(t_{0}, p_{0}, 0\right), \text { bdy } \mathscr{A}\right) .
$$

Furthermore, the value in (2.11) is not zero. (Indeed, if this were not the case then $p\left(t_{0}, p_{0}, 0\right) \in$ bdy $\mathscr{A}, q\left(t_{0}, p_{0}, 0\right)=0$, and the Dissipative Condition (II) would imply that $p\left(t, p_{0}, 0\right) \notin \mathscr{A}$ for $t$ slightly to the left of $t_{0}$, which is impossible.)

Let $p_{1} \in$ bdy $\mathscr{A}$ be the point on bdy $\mathscr{A}$ nearest to $p\left(t_{0}, p_{0}, 0\right)$ (take any point if $\left.p\left(t_{0}, p_{0}, 0\right)=0\right)$, and set

$$
\rho(t)=p\left(t, p_{0}, 0\right)-p_{1}, \quad \sigma(t)=q\left(t, p_{0}, 0\right) .
$$

Clearly $p\left(t_{0}, p_{0}, 0\right)=\mu p_{1}$ for some $\mu \in[0,1)$, and so

$$
\rho\left(t_{0}\right)=-(1-\mu) p_{1} \text {. }
$$


We consider the functions $\rho$ and $\sigma$ on $\left[0, t_{0}\right]$. One has there

$$
\left\{\begin{aligned}
\rho^{\prime}(t) & =F\left(p_{1}+\rho(t), \sigma(t)\right) \\
& =\left[\int_{0}^{1} D F\left(p_{1}+\theta \rho(t), \theta \sigma(t)\right) d \theta\right](\rho(t), \sigma(t))+F\left(p_{1}, 0\right), \\
\sigma^{\prime}(t) & =-A \sigma(t)+G\left(p_{1}+\rho(t), \sigma(t)\right) \\
& =-A \sigma(t)+\left[\int_{0}^{1} D G\left(p_{1}+\theta \rho(t), \theta \sigma(t)\right) d \theta\right](\rho(t), \sigma(t)) .
\end{aligned}\right.
$$

Consider the Lyapunov function $V$ given in the Cone Condition $(\mathrm{V})$. The definitions of $t_{0}$ and $p_{1}$ imply that

$$
\begin{aligned}
& V(\rho(t), \sigma(t))<0 \quad \text { in }\left(0, t_{0}\right), \\
& V\left(\rho\left(t_{0}\right), \sigma\left(t_{0}\right)\right)=0 .
\end{aligned}
$$

By using the Dirichlet Condition (II) and (2.5), (2.12), (2.13), the time derivative $\frac{d V}{d t}$ at $t=t_{0}$ satisfies

$$
\left.\left(\frac{d V}{d t}\right)\right|_{t=t_{0}}<-\left\langle\rho\left(t_{0}\right), F\left(p_{1}, 0\right)\right\rangle=(1-\mu)\left\langle p_{1}, F\left(p_{1}, 0\right)\right\rangle<0
$$

The inequality (2.15) now contradicts (2.14), which completes the proof.

One consequence of the last lemma is that any solution beginning in $\mathscr{P} \times\{0\}$ stays in $\mathscr{G}$ for $t>0$, as we now show.

Lemma 2.2. If $p_{0} \in \mathscr{P}$ then $u\left(t, p_{0}, 0\right) \in \mathscr{G}$ for all $t>0$, and hence $\mathfrak{M}_{t} \subset \mathscr{G}$ for all $t>0$.

Proof. If $p_{0} \in \mathscr{A}$ then $u\left(t, p_{0}, 0\right) \in \mathscr{E}$ for all $t>0$, by Lemma 2.1. If $p_{0} \in \mathscr{P} \backslash \mathscr{A}$ then by the Dissipative Condition (II),

$$
u\left(t, p_{0}, 0\right)=\left(p\left(t, p_{0}, 0\right), 0\right) \in(\mathscr{P} \backslash \mathscr{A}) \times\{0\} \quad \text { for } 0<t<t_{0}
$$

where $t_{0}$ is the first time for which $p\left(t_{0}, p_{0}, 0\right) \in$ bdy $\mathscr{A}$. By Lemma 2.1, for $t>t_{0}$ one has $u\left(t, p_{0}, 0\right) \in \mathscr{E}$. Since $u\left(t, p_{0}, 0\right)$ is a typical point of $\mathfrak{M}_{t}$, the proof is complete.

There are a few elementary facts we will need later in the argument. Let $u_{1}=\left(p_{1}, q_{1}\right)$ and $u_{2}=\left(p_{2}, q_{2}\right)$ be distinct points in $\mathscr{G}$ and assume that $u_{1} \notin \mathscr{A} \times \mathscr{C}$. Then one has $q_{1}=0,\left\|p_{1}\right\|>R_{1}$ and we claim that

$$
\left\|q_{1}-q_{2}\right\|<\left\|p_{1}-p_{2}\right\| \text {. }
$$

Indeed if $u_{2} \notin \mathscr{A} \times \mathscr{C}$, then $q_{2}=0$ and (2.16) follows from the fact that $u_{1} \neq u_{2}$. On the other hand, if $u_{2} \in \mathscr{A} \times \mathscr{C}$, then $u_{2} \in \mathscr{E}$ and with $\mu$ chosen so that $0<\mu<1$ and $\mu p_{1} \in$ bdy $\mathscr{A}$ one has

$$
\frac{\left\|q_{1}-q_{2}\right\|}{\left\|p_{1}-p_{2}\right\|}=\frac{\left\|q_{2}\right\|}{\left\|p_{1}-p_{2}\right\|}<\frac{\left\|q_{2}\right\|}{\left\|\mu p_{1}-p_{2}\right\|} \leq \frac{\left\|q_{2}\right\|}{\operatorname{dist}\left(p_{2}, \operatorname{bdy} \mathscr{A}\right)} \leq 1 \text {. }
$$


Let $u_{j}(t)=\left(p_{j}(t), q_{j}(t)\right), j=1,2$ be two solutions of $(2.1)$ with $u_{j}(t) \in \mathscr{G}$, for $t \in\left[t_{0}, t_{1}\right]$, and $j=1,2$. We claim that there is a unique $t_{2} \in\left[t_{0}, t_{1}\right]$ such that one has

$$
u_{j}(t) \in \mathscr{E} \subset \mathscr{A} \times \mathscr{C}, \quad t \in\left(t_{2}, t_{1}\right], j=1,2,
$$

and for at least one solution, say $u_{1}(t)$, one has

$$
u_{1}(t) \in(\mathscr{P} \backslash \mathscr{A}) \times\{0\}, \quad t \in\left[t_{0}, t_{2}\right) .
$$

The proof of (2.17) and (2.18) follows immediately from Lemma 2.1 and the observation that the Dissipative Condition implies that the points on bdy $\mathscr{A} \times$ $\{0\}$ are strict ingress points to $\mathscr{A} \times \mathscr{C}$. (Generally, $t_{2}$ is the first time in $\left[t_{0}, t_{1}\right]$ at which both $u_{1}(t)$ and $u_{2}(t)$ belong to $\mathscr{E}$, if such exists. If both these solutions belong to $\mathscr{E}$ for all $t \in\left[t_{0}, t_{1}\right]$, then $t_{2}=t_{0}$ and $(2.18)$ is vacuous, while if both solutions lie outside $\mathscr{E}$ for all $t \in\left[t_{0}, t_{1}\right]$, then $t_{2}=t_{1}$ and (2.17) is vacuous.) Furthermore, we note that if $u_{1}(t)$ and $u_{2}(t)$ are distinct solutions, it then follows from (2.16) and (2.18) that

$$
\left\|q_{1}(t)-q_{2}(t)\right\|<\left\|p_{1}(t)-p_{2}(t)\right\|, \quad t \in\left[t_{0}, t_{2}\right) .
$$

Lemma 2.3. Let $u_{j}(t)=\left(p_{j}(t), q_{j}(t)\right)$, for $j=1,2$, be two solutions of $(2.1)$ for $t$ in some interval $\left[t_{0}, t_{1}\right]$, and suppose that

$$
\begin{gathered}
u_{j}(t) \in \mathscr{A} \times \mathscr{C} \quad \text { in }\left[t_{0}, t_{1}\right], j=1,2 ; \\
\left\|q_{1}\left(t_{0}\right)-q_{2}\left(t_{0}\right)\right\|<\left\|p_{1}\left(t_{0}\right)-p_{2}\left(t_{0}\right)\right\| .
\end{gathered}
$$

Then for each $t \in\left(t_{0}, t_{1}\right]$, either

$$
\left\|q_{1}(t)-q_{2}(t)\right\|<\left\|p_{1}(t)-p_{2}(t)\right\|
$$

or else

$$
u_{1}(t)=u_{2}(t)
$$

Moreover, the same conclusions are valid if the first condition in (2.20) is replaced by

$$
u_{j}(t) \in \mathscr{G} \quad \text { in }\left[t_{0}, t_{1}\right], j=1,2 .
$$

Proof. If (2.22) holds for one value of $t \in\left[t_{0}, t_{1}\right]$, then it holds for all larger values of $t$ in $\left[t_{0}, t_{1}\right]$. Therefore we can assume that (2.22) fails for all $t \in$ $\left[t_{0}, t_{1}\right]$. Let $\rho=p_{1}-p_{2}$ and $\sigma=q_{1}-q_{2}$. Define $J$ by

$$
J=\left\{t \in\left[t_{0}, t_{1}\right]:\|\sigma(t)\|=\|\rho(t)\|\right\} .
$$

If $J$ is empty then it follows from the second part of (2.20) that $\|\sigma(t)\|<\|\rho(t)\|$ for all $t \in\left[t_{0}, t_{1}\right]$. We will now prove that $J$ must be empty. (Note that because of the second inequality in (2.20), one has $t_{0} \notin J$.)

Assume now that $u_{j}(t) \in \mathscr{A} \times \mathscr{C}$ in $\left[t_{0}, t_{1}\right], j=1,2$. From (2.5) one has that $V^{\prime}(t)<0$ for every $t \in J$ where $V^{\prime}=\left\langle\sigma, \sigma^{\prime}\right\rangle-\left\langle\rho, \rho^{\prime}\right\rangle$. This means that for every $\tau \in J$

$$
\|\sigma(t)\|-\|\rho(t)\|
$$


is strictly decreasing in a neighborhood of $\tau$. If $J$ is not empty then there is a $\tau \in J$ with $t_{0}<\tau$. Then $\|\sigma(t)\|-\|\rho(t)\|$ is positive to the left of $\tau$. Since $\left\|\sigma\left(t_{0}\right)\right\|-\left\|\rho\left(t_{0}\right)\right\| \leq 0$, this means that there is a $t_{3} \in J$ such that $t_{0}<t_{3}<\tau$ and

$$
\|\sigma(t)\|-\|\rho(t)\|>0, \quad t_{3}<t<\tau,
$$

which contradicts the fact that $V^{\prime}\left(t_{3}\right)<0$.

Finally assume that $u_{j}(t) \in \mathscr{G}$ for all $t \in\left[t_{0}, t_{1}\right], j=1,2$, and let $t_{2} \in$ $\left[t_{0}, t_{1}\right]$ be fixed so that $(2.17),(2.18)$, and (2.19) are valid. It follows from (2.19) that $\left\|\sigma\left(t_{2}\right)\right\| \leq\left\|\rho\left(t_{2}\right)\right\|$. The argument of the last paragraph applies on the interval $\left(t_{2}, t_{1}\right]$ and consequently $\|\sigma(t)\|<\|\rho(t)\|$ for $t_{2}<t<t_{1}$. Also (2.19) implies that $\|\sigma(t)\|<\|\rho(t)\|$ for $t_{0}<t<t_{2}$. At $t=t_{2}$ one must have $\left\|\sigma\left(t_{2}\right)\right\|<\left\|\rho\left(t_{2}\right)\right\|$, or else $t_{2}=t_{0}$. The argument is that if both these fail then both $u_{1}\left(t_{2}\right)$ and $u_{2}\left(t_{2}\right)$ are in $\mathscr{A} \times \mathscr{C}$. Hence $V^{\prime}\left(t_{2}\right)<0$, which contradicts (2.19).

The next corollary follows immediately from the above two results and the definition of $\mathfrak{M}_{t}$.

Lemma 2.4. If $\left(p_{1}, q_{1}\right)$ and $\left(p_{2}, q_{2}\right)$ are distinct points of $\mathfrak{M}_{t}$ for some $t>0$, then

$$
\left\|q_{1}-q_{2}\right\|<\left\|p_{1}-p_{2}\right\| \text {. }
$$

As a result we next conclude that each $\mathfrak{M}_{t}$ is the graph of a Lipschitz continuous function.

Lemma 2.5. For each $t_{0}>0$ there exists a function $\Phi_{t_{0}}: \mathscr{P} \rightarrow \mathscr{Q}$ such that

$$
\mathfrak{M}_{t_{0}}=\operatorname{graph} \Phi_{t_{0}}
$$

\section{Furthermore}

$$
\begin{gathered}
\operatorname{supp} \Phi_{t_{0}} \subset \mathscr{A}, \\
\Phi_{t_{0}}(p) \in \mathscr{C} \quad \text { for } p \in \mathscr{P}, \\
\left\|\Phi_{t_{0}}\left(p_{1}\right)-\Phi_{t_{0}}\left(p_{2}\right)\right\|<\left\|p_{1}-p_{2}\right\| \quad \text { for } p_{1}, p_{2} \in \mathscr{P}, p_{1} \neq p_{2} .
\end{gathered}
$$

Proof. Assume for the moment that for each $p_{1} \in \mathscr{P}$ and $t_{0}>0$ there exists $q_{1} \in \mathscr{Q}$ such that $\left(p_{1}, q_{1}\right) \in \mathfrak{M}_{t_{0}}$. The uniqueness of $q_{1}$ and hence the existence of $\Phi_{t_{0}}$ satisfying (2.23) follow from Lemma 2.4. Also, the properties (2.24), (2.25), and (2.26) follow from Lemmas 2.2 and 2.3 and the definition of $\mathscr{G}$. (Note that $\mathscr{G} \subset \mathscr{P} \times \mathscr{C}$.)

Let $p_{1} \in \mathscr{P}$ and $t_{0}>0$ be given. We will show that $p_{1}=p\left(t_{0}, p_{0}, 0\right)$ for some $p_{0} \in \mathscr{P}$; this gives $\left(p_{1}, q_{1}\right) \in \mathfrak{M}_{t_{0}}$ with $q_{1}=q\left(t_{0}, p_{0}, 0\right)$, as required. Fix $R>2 e^{t_{0}} \max \left\{\left\|p_{1}\right\|, R_{1}\right\}$ and let $\mathscr{B}$ denote the ball

$$
\mathscr{B}=\left\{p_{0} \in \mathscr{P}:\left\|p_{0}\right\|<R\right\} .
$$

Observe that by the Dissipative Condition (II) and (2.8) one has

$$
p\left(t, p_{0}, 0\right)=e^{-t} p_{0}, \quad q\left(t, p_{0}, 0\right)=0
$$


for $t \in\left[0, t_{0}\right]$ whenever $p_{0} \in$ bdy $\mathscr{B}$. Consequently

$$
p_{1} \notin p(t, \text { bdy } \mathscr{B}, 0)
$$

for $t \in\left[0, t_{0}\right]$. Therefore the degree

$$
\operatorname{deg}\left(p(t, \cdot, 0), \mathscr{B}, p_{1}\right)
$$

of the map $p(t, \cdot, 0)$ in $\mathscr{B}$ with respect to the point $p_{1}$ is well defined and independent of $t$. As $p_{1} \in \mathscr{B}$ and $p(0, \cdot, 0)$ is the identity map, it follows that the degree in (2.27) equals 1 . This implies that $p_{1} \in p\left(t_{0}, \mathscr{B}, 0\right)$ hence there is a $p_{0} \in \mathscr{B}$ with $p_{1}=p\left(t_{0}, p_{0}, 0\right)$ as required.

In the sequel we shall let $\Phi_{t}$ denote the function satisfying $\mathfrak{M}_{t}=\operatorname{graph} \Phi_{t}$, for $t>0$.

\subsection{The behavior as $t \rightarrow \infty$.}

Lemma 2.6. Let $u_{j}(t)=\left(p_{j}(t), q_{j}(t)\right), j=1,2$, be two solutions of (2.1) for $t$ in some interval $\left[t_{0}, t_{1}\right]$, and suppose that either

$$
u_{j}(t) \in \mathscr{A} \times \mathscr{C}, \quad t \in\left[t_{0}, t_{1}\right], \quad j=1,2,
$$

or

$$
u_{j}(t) \in \mathscr{G}, \quad t \in\left[t_{0}, t_{1}\right], \quad j=1,2 .
$$

In addition assume that

$$
\left\|p_{1}\left(t_{1}\right)-p_{2}\left(t_{1}\right)\right\|<\left\|q_{1}\left(t_{1}\right)-q_{2}\left(t_{1}\right)\right\|, \quad u_{1}\left(t_{1}\right) \neq u_{2}\left(t_{1}\right) .
$$

Then

$$
\left\|p_{1}(t)-p_{2}(t)\right\|<\left\|q_{1}(t)-q_{2}(t)\right\| \leq e^{-(\Lambda-2 \gamma)\left(t-t_{0}\right)}\left\|q_{1}\left(t_{0}\right)-q_{2}\left(t_{0}\right)\right\|
$$

for $t_{0} \leq t<t_{1}$.

Proof. The first inequality in (2.29) is essentially a restatement of Lemma 2.3. Set $\rho(t)=p_{1}(t)-p_{2}(t)$ and $\sigma(t)=q_{1}(t)-q_{2}(t)$ as before. Assume that $u_{j}(t) \in \mathscr{A} \times \mathscr{C}$ for $t \in\left[t_{0}, t_{1}\right], j=1,2$. Then from the differential equation (2.4), the Linear Stability Condition (IV), and the fact that $\|\rho(t)\| \leq\|\sigma(t)\|$ one finds that

$$
\begin{aligned}
\frac{d}{d t}\|\sigma(t)\|^{2} & =2\left\langle\sigma(t), \sigma^{\prime}(t)\right\rangle \\
& =-2\langle\sigma(t), A \sigma(t)\rangle+2\left\langle\sigma(t), G\left(p_{1}(t), q_{1}(t)\right)-G\left(p_{2}(t), q_{2}(t)\right)\right\rangle \\
& \leq-2 \Lambda\|\sigma(t)\|^{2}+2 \gamma\|\sigma(t)\|\{\|\rho(t)\|+\|\sigma(t)\|\} \\
& \leq-2(\Lambda-2 \gamma)\|\sigma(t)\|^{2} .
\end{aligned}
$$

Integrating this differential inequality yields the desired result.

In case $u_{j}(t) \in \mathscr{G}$ for $t \in\left[t_{0}, t_{1}\right], j=1,2$, then inequality (2.28) and the first inequality of (2.29) (which again follows from Lemma 2.3) together imply that $u_{j}(t) \in \mathscr{A} \times \mathscr{C}$ for all $t \in\left[t_{0}, t_{1}\right], j=1,2$. Therefore, this reduces matters to the previous case. 
One application of Lemma 2.6 occurs when

$$
p=p_{1}=p_{2} \in \mathscr{A} \quad \text { and } \quad q_{1}=\Phi_{t_{1}}\left(p_{1}\right), q_{2}=\Phi_{t_{2}}\left(p_{2}\right)
$$

where $0 \leq t_{1}<t_{2}$. Let $u_{j}(t)=\left(p_{j}(t), q_{j}(t)\right)$ denote the solution of $(2.1)$ that satisfies $u_{j}\left(t_{1}\right)=\left(p_{j}, q_{j}\right), j=1,2$. If $q_{1} \neq q_{2}$ then the hypotheses of Lemma 2.6 are satisfied on $\left[0, t_{1}\right]$. Consequently from (2.29) one has

$$
\left\|q_{1}(t)-q_{2}(t)\right\| \leq e^{-(\Lambda-2 \gamma) t}\left\|q_{1}(0)-q_{2}(0)\right\|
$$

for $0 \leq t<t_{1}$. Since $q_{1}(0), q_{2}(0) \in \mathscr{C}$ by Lemma 2.2 , we obtain the following result.

Lemma 2.7. One has

$$
\left\|\Phi_{t_{1}}(p)-\Phi_{t_{2}}(p)\right\| \leq e^{-(\Lambda-2 \gamma) t_{1}}(\operatorname{diam} \mathscr{C}), \quad t_{2}>t_{1}>0,
$$

for all $p \in \mathscr{P}$.

Since (2.30) implies that $\Phi_{t}$ satisfies a Cauchy condition as $t \rightarrow \infty$, we have the following result, which asserts the existence of a limiting function $\Phi(p)$.

Lemma 2.8. The limit, $\lim \Phi_{t}=\Phi,($ as $t \rightarrow \infty)$ exists uniformly for some function $\Phi: \mathscr{P} \rightarrow \mathscr{Q}$. In fact one has:

$$
\begin{aligned}
& \left\|\Phi_{t}(p)-\Phi(p)\right\|<e^{-(\Lambda-2 \gamma) t}(\operatorname{diam} \mathscr{C}), \quad \text { for } p \in \mathscr{P}, \\
& \operatorname{supp} \Phi \subset \mathscr{A}, \\
& \Phi(p) \in \mathscr{C} \quad \text { for } p \in \mathscr{P}, \\
& \operatorname{graph} \Phi \subset \mathscr{G}, \\
& \left\|\Phi\left(p_{1}\right)-\Phi\left(p_{2}\right)\right\| \leq\left\|p_{1}-p_{2}\right\| \quad \text { for } p_{1}, p_{2} \in \mathscr{P} .
\end{aligned}
$$

We define the manifold $\mathfrak{M}$ by $\mathfrak{M}=\operatorname{graph} \Phi$, where $\Phi$ is given by the above lemma. As we will now see, $\mathfrak{M}$ is an invariant manifold for (2.1) and it satisfies the conclusions of Theorem A. Since one has $\Phi=\lim \Phi_{t}$, one sometimes writes this as $\mathfrak{M}=\lim \mathfrak{M}_{t}$; see Figure 2 .

2.7. The invariance and stability of $\mathfrak{M}$. The next step is to study $\operatorname{dist}(u(t), \mathfrak{M})$ for solutions $u(t)$ of $(2.1)$ which are in $\mathscr{A} \times \mathscr{C}$. As a result we will establish the invariance of $\mathfrak{M}$ as well as its local stability.

Lemma 2.9. $\mathfrak{M}$ is invariant. That is, if $u(t)=(p(t), q(t))$ is a solution of $(2.1)$ that satisfies $q\left(t_{0}\right)=\Phi\left(p\left(t_{0}\right)\right)$ for some $t_{0}$, then $u(t)$ can be defined for all $t \in R$ so that one has $q(t)=\Phi(p(t))$ for all $t \in R$. Furthermore $p(t)$ satisfies the ordinary differential equation

$$
p^{\prime}=F(p, \Phi(p))
$$

on $R$. Conversely if $p(t)$ is a solution of $(2.31)$ then $q(t)=\Phi(p(t))$ is a solution of

$$
q^{\prime}=-A q+G(p(t), q)
$$

and $u(t)=(p(t), \Phi(p(t)))$ is a solution of $(2.1)$. 
Proof. Let $u_{\tau}=\left(p_{\tau}, q_{\tau}\right) \in \mathfrak{M}_{\tau}$ for some $\tau \geq 0$. It then follows from the definition of $\mathfrak{M}_{\tau}$ and the Dissipative Condition (II) that the solution $u_{\tau}(t)=$ $\left(p_{\tau}(t), q_{\tau}(t)\right)$ of $(2.1)$ that satisfies $u(0)=u_{\tau}$ is defined for all $t$ in the interval $[-\tau,+\infty)$ and satisfies $u_{\tau}(t) \in \mathfrak{M}_{\tau+t}$ on this interval. In particular one has

$$
q_{\tau}(t)=\Phi_{\tau+t}\left(p_{\tau}(t)\right)
$$

on this interval. Now fix $p \in \mathscr{P}$, and set $p_{\tau}=p$; then $q_{\tau}=\Phi_{\tau}(p)$. Letting $\tau$ vary, we have that $\Phi_{\tau}(p) \rightarrow \Phi(p)$, as $\tau \rightarrow \infty$, and the solution $u_{\tau}(t)$ converges (uniformly on compact sets in $R$ ) to the solution $u(t)=(p(t), q(t))$ with $u(0)=(p, \Phi(p))$. (The convergence follows from the equicontinuity of $q_{\tau}(t)$, which in turn follows from the bound $\left\|A q_{\tau}(t)\right\| \leq R_{2}$. As is usual, we take limits in the integrated form of the equation (2.1).) Consequently the limits (as $\tau \rightarrow \infty)$ in (2.32) exists and one gets $q(t)=\Phi(p(t))$ for all $t \in R$.

The solution $u_{\tau}(t)$ satisfies

$$
\begin{aligned}
& p_{\tau}^{\prime}(t)=F\left(p_{\tau}(t), \Phi_{\tau+t}\left(p_{\tau}(t)\right)\right), \\
& q_{\tau}^{\prime}(t)=-A q_{\tau}(t)+G\left(p_{\tau}(t), q_{\tau}(t)\right) .
\end{aligned}
$$

By letting $\tau \rightarrow \infty$ the first equation converges to (2.31) and the second equation converges to

$$
q^{\prime}(t)=-A q(t)+G(p(t), q(t)) .
$$

Hence $p(t)$ is a solution of (2.31). Conversely, if $p(t)$ is a solution of (2.31) then clearly $u(t)=(p(t), \Phi(p(t)))$ is a solution of $(2.1)$.

The differential equation (2.31), which is called an inertial form in Foias, Sell, and Temam (1986), is another illustration of the Pliss Reduction Principle, Pliss (1964).

Lemma 2.10. Let $u(t)=(p(t), q(t))$ be a solution of (2.1) with $u(t)$ in $\mathscr{E}$ for all $t$ in some interval $\left[t_{0}, t_{1}\right]$. Then one has

$$
\operatorname{dist}(u(t), \mathfrak{M}) \leq 2 e^{-(\Lambda-2 \gamma)\left(t-t_{0}\right)}(\operatorname{diam} \mathscr{C})
$$

for $t_{0} \leq t \leq t_{1}$.

Proof. We will use Lemma 2.6. First observe that without loss of generality we may assume that $u(t) \notin \mathfrak{M}$ for all $t \in\left[t_{0}, t_{1}\right]$. This is because the invariance of $\mathfrak{M}$ and the uniqueness in forward time imply that if $u\left(t_{2}\right) \in \mathfrak{M}$ for some $t_{2}$, then $u(t) \in \mathfrak{M}$ for all $t \geq t_{2}$. Let $u_{1}(t)=\left(p_{1}(t), q_{1}(t)\right)$ be the solution given in the hypotheses of Lemma 2.10. We define the second solution $u_{2}(t)=$ $\left(p_{2}(t), q_{2}(t)\right)$ as that solution of $(2.1)$ that satisfies $u_{2}\left(t_{1}\right)=\left(p_{1}\left(t_{1}\right), \Phi\left(p_{1}\left(t_{1}\right)\right)\right)$. By Lemmas 2.8 and 2.9 one has $u_{2}(t) \in \mathfrak{M} \subset \mathscr{G}$ for all $t \in R$. As $p_{1}\left(t_{1}\right)=$ $p_{2}\left(t_{1}\right)$ and $u_{1}\left(t_{1}\right) \neq u_{2}\left(t_{1}\right)$, the hypotheses of Lemma 2.6 hold, and therefore, 
for $t \in\left[t_{0}, t_{1}\right]$, one has

$$
\begin{aligned}
\operatorname{dist}\left(u_{1}(s), \mathfrak{M}\right) & \leq\left\|u_{1}(s)-u_{2}(s)\right\| \\
& \leq\left\|p_{1}(t)-p_{2}(t)\right\|+\left\|q_{1}(t)-q_{2}(t)\right\| \\
& \leq 2\left\|q_{1}(s)-q_{2}(s)\right\| \\
& \leq 2 e^{-(\Lambda-2 \gamma)\left(s-t_{0}\right)}\left\|q_{1}\left(t_{0}\right)-q_{2}\left(t_{0}\right)\right\| .
\end{aligned}
$$

Since $\left\|q_{1}\left(t_{0}\right)-q_{2}\left(t_{0}\right)\right\| \leq \operatorname{diam} \mathscr{C},(2.33)$ is valid.

2.8. Smoothness of $\mathfrak{M}$. In this section we shall prove that the invariant manifold $\mathfrak{M}$ is a $C^{1}$-manifold provided the differential equation (2.1) satisfies the following stronger Cone Condition: ${ }^{7}$

(Va)(Uniform Cone Condition). Let $V \stackrel{\text { def }}{=} \frac{1}{2}\|\sigma\|^{2}-\frac{1}{2}\|\rho\|^{2}$ and $V^{\prime} \stackrel{\text { def }}{=}\left\langle\sigma, \sigma^{\prime}\right\rangle-$ $\left\langle\rho, \rho^{\prime}\right\rangle$ where $\rho \in \mathscr{P}, \sigma \in \mathscr{D} \subset \mathscr{Q}$ and $\rho^{\prime}$ and $\sigma^{\prime}$ are given by the variational equation (2.2). Then there is a $\xi>0$ such that for all $u \in \mathscr{A} \times \mathscr{C}$ and $\rho \in$ $\mathscr{P}, \sigma \in \mathscr{D} \subset \mathscr{Q}$ with $\|\rho\|=\|\sigma\|=1$ one has

$$
V^{\prime}=\langle\sigma,-A \sigma+D G(u)(\rho, \sigma)\rangle-\langle\rho, D F(u)(\rho, \sigma)\rangle \leq-\xi .
$$

In order to show that the invariant manifold $\mathfrak{M}=\operatorname{graph} \Phi$ is smooth we fix $\tau>0$ and consider the sequence of functions $\Phi_{n \tau}$ given by Lemma 2.5 for $t_{0}=n \tau$. The quantity $\tau$ need only be sufficiently small, and will remain fixed throughout this section. As shown in Lemma 2.8 one has

$$
\left\|\Phi_{n \tau}\left(p_{0}\right)-\Phi\left(p_{0}\right)\right\| \leq e^{-(\Lambda-2 \gamma) n \tau}(\operatorname{diam} \mathscr{C})
$$

for $p_{0} \in \mathscr{P}$. We will now show, by induction, that each element in the sequence $\left\{\Phi_{n}\right\}$ is a $C^{1}$-function of $p_{0} \in \mathscr{P}$. For this purpose we shall use the notation

$$
\Psi_{n}\left(p_{0}\right)=\frac{\partial}{\partial p_{0}} \Phi_{n \tau}\left(p_{0}\right)
$$

when $\Phi_{n \tau}$ is $C^{1}$. Here, $\Psi_{n}\left(p_{0}\right) \in \mathscr{L}=\mathscr{L}(\mathscr{P}, \mathscr{Q})$, that is, $\Psi_{n}: \mathscr{P} \rightarrow$ $\mathscr{L}(\mathscr{P}, \mathscr{Q})$ is the Fréchet derivative of $\Phi_{n \tau}$. Note also that $\left\|\Psi_{n}\left(p_{0}\right)\right\|_{\mathscr{L}} \leq 1$ by (2.26). Since $\Phi_{0}\left(p_{0}\right) \equiv 0$ one has $\Psi_{0}\left(p_{0}\right) \equiv 0$. Now assume that $\Phi_{n \tau}$ is $C^{1}$, and $\Psi_{n}$ is given by (2.35). Then $\Phi_{(n+1) \tau}$ satisfies

$$
\Phi_{(n+1) \tau}\left(p_{1}\right)=q_{1}
$$

where $p_{1}=p\left(\tau, p_{0}, \Phi_{n \tau}\left(p_{0}\right)\right)$ and $q_{1}=q\left(\tau, p_{0}, \Phi_{n \tau}\left(p_{0}\right)\right)$. Let $\rho(t)$ and $\sigma(t)$ satisfy

$$
\rho(t)=\frac{\partial}{\partial p_{0}} p\left(t, p_{0}, \Phi_{n \tau}\left(p_{0}\right)\right), \quad \sigma(t)=\frac{\partial}{\partial p_{0}} q\left(t, p_{0}, \Phi_{n \tau}\left(p_{0}\right)\right) .
$$

\footnotetext{
${ }^{7}$ In finite dimensional Hilbert spaces the two cone conditions are the same.
} 
Then $\rho(0)=I, \sigma(0)=\Psi_{n}\left(p_{0}\right), \rho(\tau)=\partial p_{1} / \partial p_{0}$ and $\sigma(\tau)=\partial q_{1} / \partial p_{0}$. By applying the chain rule to $(2.36)$ we obtain

$$
\begin{aligned}
\Psi_{n+1}\left(p_{1}\right) & =\frac{\partial}{\partial p_{1}} \boldsymbol{\Phi}_{(n+1) \tau}\left(p_{1}\right)=\frac{\partial}{\partial p_{1}} q_{1} \\
& =\left[\frac{\partial}{\partial p_{0}} q_{1}\right]\left[\frac{\partial}{\partial p_{1}} p_{0}\right]=\sigma(\tau) \rho(\tau)^{-1},
\end{aligned}
$$

thus $\Psi_{n+1}\left(p_{1}\right)$ is a continuous function of $p_{1}$. Furthermore, if $\rho(t) \alpha$ is any solution of the $\rho$-equation in (2.2) then $\sigma(t) \alpha=\sigma(t) \rho^{-1}(t) \rho(t) \alpha$ is a solution of the $\sigma$-equation in (2.2). Since $\|\sigma(0) \alpha\| \leq\|\rho(0) \alpha\|=\|\alpha\|$ the cone condition implies that $\|\sigma(t)\| \leq\|\rho(t)\|$ for $0 \leq t$. Hence $\left\|\sigma(t) \rho(t)^{-1}\right\|_{\mathscr{L}} \leq 1$ and consequently $\left\|\Psi_{n+1}\left(p_{1}\right)\right\|_{\mathscr{L}} \leq 1$ for all $p_{1} \in \mathscr{P}$.

For $n \geq 1$ we let $u_{n}\left(t, p_{0}\right)=\left(p_{n}\left(t, p_{0}\right), q_{n}\left(t, p_{0}\right)\right)$ denote the solution of (2.1) satisfying the condition

$$
u_{n}\left(\tau, p_{0}\right)=\left(p_{0}, \Phi_{n \tau}\left(p_{0}\right)\right) \quad p_{0} \in \mathscr{P} .
$$

Next define

$$
e_{n, m}=\sup \left\{\left\|u_{n}\left(t, p_{0}\right)-u_{m}\left(t, p_{0}\right)\right\|: 0 \leq t \leq \tau, p_{0} \in \mathscr{P}\right\} .
$$

Lemma 2.11. With $e_{n, m}$ defined as above one has

$$
\lim _{m, n \rightarrow \infty} e_{n, m}=0 \text {. }
$$

Proof. By using the facts that $F(p, 0)=-p$ for $p \in \mathscr{P} \backslash 2 \mathscr{A}$ and that $F$ is $C^{1}$ in $\mathscr{A} \times \mathscr{C}$ it is easy to verify that there is a constant $K$ such that whenever $u_{1}, u_{2} \in \mathscr{G}$ then

$$
\left\|F\left(u_{1}\right)-F\left(u_{2}\right)\right\| \leq K\left\|u_{1}-u_{2}\right\| .
$$

With $\varepsilon>0$ given we use Lemma 2.8 to fix $T=T(\varepsilon)$ so that

$$
\left\|\Phi_{t}(p)-\Phi(p)\right\|<\varepsilon \quad p \in \mathscr{P}, t \geq T .
$$

Assume now that $n$ is large enough that $(n-1) \tau \geq T$. It follows from (2.32) that

$$
q_{n}\left(t, p_{0}\right)=\Phi_{(n-1) \tau+t}\left(p_{n}\left(t, p_{0}\right)\right), \quad p_{0} \in \mathscr{P}, t \geq 0,
$$

hence (2.38) implies that

$$
\left\|q_{n}\left(t, p_{0}\right)-\Phi\left(p_{n}\left(t, p_{0}\right)\right)\right\| \leq \varepsilon, \quad p_{0} \in \mathscr{P}, t \geq 0 .
$$

Furthermore Lemma 2.9 also implies that $p_{n}(t)=p_{n}\left(t, p_{0}\right)$ is a solution of

$$
p_{n}^{\prime}(t)=F\left(p_{n}(t), \Phi\left(p_{n}(t)\right)\right)+H_{n}(t)
$$

where $H_{n}(t)=F\left(p_{n}(t), \Phi_{(n-1) \tau+t}\left(p_{n}(t)\right)\right)-F\left(p_{n}(t), \Phi\left(p_{n}(t)\right)\right)$ satisfies

$$
\left\|H_{n}(t)\right\| \leq K \varepsilon, \quad p_{0} \in \mathscr{P}, t \geq 0,
$$

by (2.37) and (2.38). 
Next let $u(t)=(p(t), q(t))$ denote the solution of (2.1) satisfying, at time $t=\tau, u(\tau)=\left(p_{0}, \Phi\left(p_{0}\right)\right)$ with the same $p_{0}$ as above. It then follows from Lemma 2.9 that $p(t)$ is a solution of $(2.31)$. Upon setting $\Delta(t)=\left\|p_{n}(t)-p(t)\right\|$ one has $\Delta(\tau)=0$ and hence

$$
\begin{aligned}
\Delta(t) & \leq \int_{t}^{\tau}\left\|F\left(p_{n}(s), \Phi\left(p_{n}(s)\right)\right)-F(p(s), \Phi(p(s)))+H_{n}(s)\right\| d s \\
& \leq \int_{t}^{\tau}(2 K \Delta(s)+K \varepsilon) d s
\end{aligned}
$$

for $0 \leq t \leq \tau$. Gronwall's inequality implies that

$$
\left\|p_{n}(t)-p(t)\right\| \leq K \varepsilon e^{2 K \tau}, \quad p_{0} \in \mathscr{P}, 0 \leq t \leq \tau .
$$

Next we note that

$$
\begin{aligned}
\left\|q(t)-q_{n}(t)\right\| & =\left\|\Phi(p(t))-\Phi_{(n-1) \tau+t}\left(p_{n}(t)\right)\right\| \\
& \leq\left\|\Phi(p(t))-\Phi\left(p_{n}(t)\right)\right\|+\left\|\Phi\left(p_{n}(t)\right)-\Phi_{(n-1) \tau+t}\left(p_{n}(t)\right)\right\| \\
& \leq\left\|p_{n}(t)-p(t)\right\|+\varepsilon \\
& \leq K \varepsilon e^{2 K \tau}+\varepsilon
\end{aligned}
$$

for this range of $t$. If $(k-1) \tau \geq T$ for $k=n, m$, then it follows that

$$
\left\|u_{n}\left(t, p_{0}\right)-u_{m}\left(t, p_{0}\right)\right\| \leq 4 K \varepsilon e^{2 K \tau}+2 \varepsilon
$$

for $0 \leq t \leq \tau$ and all $p_{0} \in \mathscr{P}$.

We shall use the notation

$$
D F_{n}(t)=D F\left(u_{n}\left(t, p_{0}\right)\right), \quad D G_{n}(t)=D G\left(u_{n}\left(t, p_{0}\right)\right),
$$

where $u_{n}\left(t, p_{0}\right)$ is given above.

Lemma 2.12. There is a constant $K_{0}$ with the property that for any $n \geq 0$ and for any solution $\left(\rho_{n}(t), \sigma_{n}(t)\right)$ of

$$
\begin{aligned}
& \rho^{\prime}=D F_{n}(t)(\rho, \sigma), \\
& \sigma^{\prime}=-A \sigma+D G_{n}(t)(\rho, \sigma)
\end{aligned}
$$

with $\left\|\sigma_{n}(0)\right\| \leq\left\|\rho_{n}(0)\right\| \leq 1$ one has

$$
\left\|\rho_{n}(t)\right\|+\left\|\sigma_{n}(t)\right\| \leq K_{0}\left\|\rho_{n}(0)\right\|, \quad 0 \leq t \leq \tau .
$$

Proof. The Cone Condition implies that $\left\|\sigma_{n}(t)\right\| \leq\left\|\rho_{n}(t)\right\|$ for all $t \geq 0$. Now define $K_{1}$ by

$$
K_{1}=\max \left(1, \max \left\{\|D F(u)\|_{\mathscr{L}}: u \in \mathscr{A} \times \mathscr{C}\right\}\right.
$$

One then has

$$
\begin{aligned}
\frac{1}{2} \frac{d}{d t}\left\|\rho_{n}(t)\right\|^{2} & =\left\langle\rho_{n}(t), D F_{n}(t)\left(\rho_{n}(t), \sigma_{n}(t)\right)\right\rangle \\
& \leq K_{1}\left\|\rho_{n}(t)\right\|\left\{\left\|\rho_{n}(t)\right\|+\left\|\sigma_{n}(t)\right\|\right\} \\
& \leq 2 K_{1}\left\|\rho_{n}(t)\right\|^{2}
\end{aligned}
$$


which implies that $\left\|\rho_{n}(t)\right\| \leq\left\|\rho_{n}(0)\right\| e^{2 K_{1} t} \leq K_{0}\left\|\rho_{n}(0)\right\|$ for $0 \leq t \leq \tau$, where $K_{0}=e^{2 K_{1} \tau}$.

In the next two lemmas we wish to compare two solutions $\left(\rho_{k}(t), \sigma_{k}(t)\right), k=$ $m, n$ of the linearized equation

$$
\begin{aligned}
& \rho^{\prime}=D F_{k}(t)(\rho, \sigma), \\
& \sigma^{\prime}=-A \sigma+D G_{k}(t)(\rho, \sigma),
\end{aligned}
$$

on $0 \leq t \leq \tau$, with $\rho_{k}(\tau)=\alpha$, for some $\alpha \in \mathscr{P}$, and $\sigma_{k}(\tau)=\Psi_{k}\left(p_{0}\right) \alpha$ for $k=m, n$. We define

$$
\rho(t)=\rho_{m}(t)-\rho_{n}(t), \quad \sigma(t)=\sigma_{m}(t)-\sigma_{n}(t) .
$$

Also we define $\left\|\Psi_{n}\right\|_{\infty}$ by

$$
\left\|\Psi_{n}\right\|_{\infty}=\sup \left\{\left\|\Psi_{n}\left(p_{0}\right) \alpha\right\|: p_{0} \in \mathscr{P},\|\alpha\| \leq 1\right\} .
$$

Lemma 2.13. Assume that the Uniform Cone Condition is satisfied. Then there is an $N_{0}$ such that for all $n, m \geq N_{0}$, for all $p_{0} \in \mathscr{P}$, and all $\alpha \in \mathscr{P}$, one has

$$
\|\rho(t)\| \leq\|\sigma(t)\|, \quad 0 \leq t \leq \tau .
$$

Proof. First note that without loss of generality we may take $\alpha \in \mathscr{P}$ with $\|\alpha\|=1$. Next observe that $\sigma_{n}(t)=\Psi_{n}\left(p_{n}\left(t, p_{0}\right)\right) \rho_{n}(t)$ for $0 \leq t \leq \tau$, and hence $\left\|\sigma_{n}(t)\right\| \leq\left\|\rho_{n}(t)\right\|$ there. Then an application of Gronwall's inequality to the equation for $\rho_{n}^{\prime}$ yields

$$
\left\|\rho_{n}(0)\right\| \leq e^{2 K \tau}\left\|\rho_{n}(\tau)\right\| \leq e^{2 K \tau}
$$

where $K$ is as in (2.37). Next note that $(\rho(t), \sigma(t))$ is a solution of the following system of equations:

$$
\begin{aligned}
& \rho^{\prime}=D F_{m}(t)(\rho, \sigma)+\left[D F_{m}(t)-D F_{n}(t)\right]\left(\rho_{n}(t), \sigma_{n}(t)\right), \\
& \sigma^{\prime}=-A \sigma+D G_{m}(t)(\rho, \sigma)+\left[D G_{m}(t)-D G_{n}(t)\right]\left(\rho_{n}(t), \sigma_{n}(t)\right) .
\end{aligned}
$$

Let $\Delta=\Delta(\varepsilon)$ denote the modulus of continuity of $D F(u)$ and $D G(u)$ on $(\mathscr{P} \times\{0\}) \cup(\mathscr{A} \times \mathscr{C})$. This means that $\Delta(\varepsilon)$ is a monotone nondecreasing function of $\varepsilon>0$ with $\Delta(\varepsilon) \rightarrow 0$ as $\varepsilon \rightarrow 0$ and such that

$$
\begin{gathered}
\left\|D F\left(u_{1}\right)-D F\left(u_{2}\right)\right\| \leq \Delta\left(\left\|u_{1}-u_{2}\right\|\right), \\
\left\|D G\left(u_{1}\right)-D G\left(u_{2}\right)\right\| \leq \Delta\left(\left\|u_{1}-u_{2}\right\|\right)
\end{gathered}
$$

whenever $u_{1}, u_{2} \in(\mathscr{P} \times\{0\}) \cup(\mathscr{A} \times \mathscr{C})$. We claim that (2.41) satisfies the cone condition for $n, m$ sufficiently large. Indeed from (2.34) and Lemma 2.12 one obtains (for $\|\rho\|=\|\sigma\|=1$ and $0 \leq t \leq \tau$ )

$$
\begin{aligned}
V^{\prime} & =\left\langle\sigma, \sigma^{\prime}\right\rangle-\left\langle\rho, \rho^{\prime}\right\rangle \\
& \leq-\xi+\left|\left\langle\sigma,\left[D G_{m}-D G_{n}\right]\left(\rho_{n}, \sigma_{n}\right)\right\rangle\right|+\left|\left\langle\rho,\left[D F_{m}-D F_{n}\right]\left(\rho_{n}, \sigma_{n}\right)\right\rangle\right| \\
& \leq-\xi+2 \Delta\left(e_{n, m}\right)\left\|\left(\rho_{n}, \sigma_{n}\right)\right\| \\
& \leq-\xi+2 \Delta\left(e_{n, m}\right)\left[\left\|\rho_{n}(t)\right\|+\left\|\sigma_{n}(t)\right\|\right] \\
& \leq-\xi+2 K_{0} \Delta\left(e_{n, m}\right)\left\|\rho_{n}(0)\right\| \\
& \leq-\xi+2 K_{0} e^{2 K \tau} \Delta\left(e_{n, m}\right) .
\end{aligned}
$$


Now fix $\varepsilon_{0}$ such that $\varepsilon_{0}>0$ and $\Delta\left(\varepsilon_{0}\right) \leq \xi e^{-2 K \tau} / 4 K_{0}$. We then use Lemma 2.11 to fix $N_{0}$ so that for $n, m \geq N_{0}$ one has $e_{n, m} \leq \varepsilon_{0}$. It then follows that $V^{\prime} \leq-\xi / 2$ when $n, m \geq N_{0}$, which then implies that (2.40) holds since $\|\rho(\tau)\|=0$.

Lemma 2.14. Assume that the Uniform Cone Condition holds and let $N_{0}$ be given by Lemma 2.13. Then the sequence $\left\{\Psi_{n}\right\}$ is a uniform Cauchy sequence in the norm $\left\|\Psi_{n}\right\|_{\infty}$.

Proof. Given $\eta>0$ we fix $\varepsilon>0$ so that $K_{0} e^{2 K \tau}(\Lambda-2 \gamma)^{-1} \Delta(\varepsilon) \leq \eta$. Then use Lemma 2.11 to select $N=N(\eta)$ so that $N \geq N_{0}$ and for $n, m \geq N$ one has $e_{n, m} \leq \varepsilon$.

For $n, m \geq N_{0}$ it follows from the Linear Stability Condition and Lemma 2.12 that with $(\rho(t), \sigma(t))$ as in Lemma 2.13

$$
\begin{aligned}
\|\sigma\| \frac{d}{d t}\|\sigma\| & =\frac{1}{2} \frac{d}{d t}\|\sigma\|^{2}=\left\langle\sigma, \sigma^{\prime}\right\rangle \\
& =-\langle\sigma, A \sigma\rangle+\left\langle\sigma, D G_{m}(t)(\rho, \sigma)\right\rangle+\left\langle\sigma,\left[D G_{n}(t)-D G_{m}(t)\right]\left(\rho_{n}, \sigma_{n}\right)\right\rangle \\
& \leq-\Lambda\|\sigma\|^{2}+\gamma\|\sigma\|(\|\rho\|+\|\sigma\|)+K_{0} e^{2 K \tau}\|\sigma\| \Delta\left(e_{n, m}\right) .
\end{aligned}
$$

Since $\|\rho\| \leq\|\sigma\|$, it then follows from $m, n \geq N(\eta)$ that

$$
\frac{d}{d t}\|\sigma\| \leq-\Lambda\|\sigma\|+2 \gamma\|\sigma\|+\eta(\Lambda-2 \gamma) .
$$

The Gronwall inequality then implies that

$$
\|\sigma(\tau)\| \leq \zeta\|\sigma(0)\|+\eta,
$$

where $\zeta=e^{-(\Lambda-2 \gamma) \tau}$. For $n \geq m \geq 0$ a simple induction argument yields

$$
\begin{aligned}
\left\|\Psi_{N+n}-\Psi_{N+m}\right\|_{\infty} & \leq \zeta^{m}\left\|\Psi_{N}-\Psi_{N+n-m}\right\|_{\infty}+\zeta^{m-1} \eta+\cdots+\eta, \\
& \leq 2 \zeta^{m}+\eta\left(1-\zeta^{m}\right)(1-\zeta)^{-1}
\end{aligned}
$$

where $N=N(\eta)$. As $\eta>0$ is arbitrary, the result follows from the above inequality.

Since $\Psi_{n}$ converges uniformly, the limit function $\Psi$ is continuous in $p$. Furthermore the equality

$$
\Phi_{n \tau}(p+h)-\Phi_{n \tau}(p)=\int_{0}^{1} \Psi_{n}(p+\theta h) d \theta h
$$

holds in the limit, where one has

$$
\begin{aligned}
\Phi(p+h)-\Phi(p) & =\int_{0}^{1} \Psi(p+\theta h) d \theta h \\
& =\Psi(p) h+\int_{0}^{1}[\Psi(p+\theta h)-\Psi(p)] d \theta h .
\end{aligned}
$$


Since $\Psi$ is continuous in $p$, for every $\varepsilon>0$ there is a $\delta>0$ such that

$$
\left\|\int_{0}^{1}[\Psi(p+\theta h)-\Psi(p)] d \theta h\right\| \leq \varepsilon\|h\|
$$

whenever $\|h\| \leq \delta$. This shows that $\Phi$ is $C^{1}$ and that $\Psi=D \Phi$.

We summarize this in the following statement.

Theorem B. In addition to the hypotheses of Theorem A, assume that the Uniform Cone Condition (Va) is satisfied. Then the mapping $\Phi: \mathscr{P} \rightarrow \mathscr{Q}$, for which $\mathfrak{M}=\operatorname{graph} \Phi$ is the invariant manifold for (2.1), is a $C^{1}$-function and the derivative $\Psi(p)=D \Phi(p)$ satisfies $\|\Psi\|_{\infty} \leq 1$.

We will not present the details here but one can show that when the Uniform Cone Condition is satisfied, then the invariant manifold $\mathfrak{M}$ is normally hyperbolic. The notion of normal hyperbolicity for infinite dimensional systems is closely related to the finite dimensional theory described in Sacker (1969), Fenichel (1971), Hirsch, Pugh, and Shub (1977), and Sacker and Sell (1980). The infinite dimensional theory will be developed in a forthcoming paper.

\section{VERIFICATION OF CONDITIONS (I)-(IV)}

We turn our attention now to the specific class of scalar partial differential equations of the form

$$
u_{t}=\nu \triangle u+f(x, u), \quad x \in \Omega \subset R^{n}, \quad u \in R
$$

where $\nu>0$ is a viscosity parameter and $f: \bar{\Omega} \times R \rightarrow R$ is sufficiently smooth. Throughout this section we make the standing assumption that $\Omega$ is a bounded domain with a Lipschitz boundary $\partial \Omega$. Moreover, we also assume

$$
n=\operatorname{dim} \Omega \leq 3 .
$$

Under these assumptions we demonstrate that four of the five hypotheses (all except the Uniform Cone Condition (Va)) of the Invariant Manifold Theorem hold for an equation obtained from (3.1) by modifying the nonlinearity $f$ with a suitable cut-off function. We also partially verify the Cone Condition. In later sections we specialize to the case of the rectangular and cubic domains $\Omega_{2}$ and $\Omega_{3}$ which we define as

$$
\begin{aligned}
& \Omega_{2}=\left(0,2 \pi / a_{1}\right) \times\left(0,2 \pi / a_{2}\right) \subset R^{2}, \\
& \Omega_{3}=(0,2 \pi)^{3} \subset R^{3},
\end{aligned}
$$

and show that the full Uniform Cone Condition holds. The existence of an inertial manifold in these two cases will then follow from the Invariant Manifold Theorem. 
3.1. Basic assumptions. The boundary conditions for (3.1) are assumed to be either of following:

$$
\begin{cases}\text { Dirichlet: } & u=0 \text { on } \partial \Omega \\ \text { Neumann: } & \frac{\partial u}{\partial n}=0 \text { on } \partial \Omega \\ \text { Periodic: } & u\left(t, x+\frac{2 \pi k}{a}\right)=u(t, x) \text { for each } k \in Z^{n}\end{cases}
$$

Here $2 \pi k / a \in R^{n}$ is the vector with components $2 \pi k_{j} / a_{j}$, where $a=\left(a_{1}, a_{2}\right)$ for $\Omega_{2}$ and $a=(1,1,1)$ for $\Omega_{3}$. In case $\partial \Omega$ is Lipschitz but not $C^{1}$, one evaluates $\partial u / \partial n$ almost everywhere on $\partial \Omega$ when Neumann boundary conditions are considered. Presumably, more general boundary conditions could also be taken.

We denote $L^{p}=L^{p}(\Omega)$, and $H^{s}=H^{s}(\Omega)$. In the case of periodic boundary conditions, $H^{s}(\Omega)$ consists of those functions whose periodic extensions to $R^{n}$ belong to $H_{\text {loc }}^{s}\left(R^{n}\right)$. The norm $\|\cdot\|$ will always denote the $L^{2}$ norm, while the norm in any other space $X$ will be denoted by $\|\cdot\|_{X}$ as before. For a bounded linear operator $L \in \mathscr{L}\left(L^{2}, L^{2}\right)$ we shall let $\|L\|_{\text {op }}$ denote the operator-norm $\|L\|_{\text {op }}=\sup \{\|L u\|:\|u\| \leq 1\}$.

The nonlinearity

$$
f: \bar{\Omega} \times R \rightarrow R
$$

is assumed to satisfy the following conditions for some positive constants $K_{1}$ and $K_{2}$ :

$$
\left\{\begin{array}{l}
f \text { is } C^{1} \text { in } \bar{\Omega} \times R, \\
|f(x, u)|,\left|D_{x} f(x, u)\right| \leq K_{1}|u|+K_{2} \quad \text { in } \bar{\Omega} \times R, \text { and } \\
\left|D_{u} f(x, u)\right| \leq K_{1} \quad \text { in } \bar{\Omega} \times R .
\end{array}\right.
$$

In the case of periodic boundary conditions we assume these conditions hold for the periodic extension of $f$ to $R^{n} \times R$. These assumptions will be taken for the remainder of this section. In $\S 4$ some additional smoothness will be imposed on $f$.

The assumption that $f$ satisfy the linear growth condition (3.6) near $|u|=$ $\infty$ can be made without any loss of generality when (3.1) is dissipative. For such equations the original nonlinearity $f$ may not satisfy (3.6) but a suitable modification would. In particular, if $f$ is dissipative, then there is a global attractor $\mathfrak{A} \subset L^{\infty} \cap L^{2}$ and a constant $C$ such that $\|u\|_{L^{\infty}} \leq C$ for all $u \in \mathfrak{A}$. For example, if for some $R>0$ the nonlinearity $f$ satisfies the sign condition:

$$
u f(x, u)<0 \quad \text { for all } x \in \Omega_{n} \text { and }|u| \geq R,
$$

then the maximum principle implies that

$$
\left\{u \in L^{\infty} \subset L^{2}:\|u\|_{L^{\infty}} \leq R+1\right\}
$$


is an absorbing set for (3.1). This means that the nonlinearity $f$ in (3.1) can be replaced by

$$
g(x, u)=\tilde{\phi}\left(|u|^{2}\right) f(x, u)
$$

where $\tilde{\phi}:[0, \infty) \rightarrow[0,1]$ is a smooth function satisfying $\tilde{\phi}(t)=1$ for $0 \leq t \leq$ $2 C$ and $\tilde{\phi}(t)=0$ for $4 C \leq t$. It follows that $g$ satisfies (3.6) for appropriate choice of $K_{1}$ and $K_{2}$. Furthermore the modified equation and the original equation coincide in some neighborhood of the global attractor $\mathfrak{A}^{8}$

3.2. Abstract formulation. It is clear from (3.6) that $f$ induces a map $\tilde{f}$ : $L^{2} \rightarrow L^{2}$ defined by evaluation:

$$
[\tilde{f}(u)](x)=f(x, u(x)), \quad u \in L^{2} .
$$

An easy application of the mean value theorem yields the following result.

Lemma 3.1. For $u_{1}, u_{2}$, and $u$ in $L^{2}$ one has

$$
\begin{array}{r}
\left\|\tilde{f}\left(u_{1}\right)-\tilde{f}\left(u_{2}\right)\right\| \leq K_{1}\left\|u_{1}-u_{2}\right\|, \\
\|\tilde{f}(u)\| \leq K_{1}\|u\|+K_{3},
\end{array}
$$

where $K_{3}=(\operatorname{vol} \Omega)^{1 / 2} K_{2}$.

Equation (3.1) can now be written as an abstract differential equation

$$
u^{\prime}=\nu \Delta u+\tilde{f}(u)
$$

in the phase space $\mathscr{H}=L^{2}$. In this setting $\mathscr{D}$ denotes the Laplace operator with the domain

$$
\mathscr{D}=\left\{u \in H^{2}: \text { the boundary condition (3.5) holds }\right\} \text {. }
$$

Note that $-\triangle$ is a selfadjoint, nonnegative operator with compact resolvent. As the operator $\nu \triangle$ generates an analytic semigroup in $\mathscr{H}$, one sees that for each $u_{0} \in \mathscr{H}$ the initial value problem $u(0)=u_{0}$ for $(3.8)$ possesses a unique (mild) solution. In fact this solution exists for all $t \geq 0$ by virtue of the uniform Lipschitz constant $K_{1}$ of $\tilde{f}$.

For simplicity we shall assume $\nu=1$, so the equation (3.8) in $\mathscr{H}$ takes the form

$$
u^{\prime}=\Delta u+\tilde{f}(u) .
$$

This presents no loss of generality since the case of arbitrary $\nu>0$ is treated by rescaling the time $t$ and considering the nonlinearity $\nu^{-1} f$ in place of $f$.

Let $\left\{\lambda_{m}\right\}_{m=1}^{\infty}$ denote the eigenvalues of $-\triangle$ ordered so that

$$
0 \leq \lambda_{1} \leq \lambda_{2} \leq \lambda_{3} \leq \cdots \leq \lambda_{m} \rightarrow \infty
$$

${ }^{8}$ There is an issue, however, of whether the attractor for the modified equation coincides with that for the original equation. Certainly the former contains the latter. Even if equation (3.1) does not have a global attractor in $L^{\infty}$, a modification of (3.1) so that (3.6) is satisfied can lead to useful information concerning those solutions which remain in that portion of $L^{2}$ where the original equation and the modified equation agree. 
and let $\left\{e_{1}, e_{2}, \ldots\right\}$ be a corresponding complete orthonormal set of eigenfunctions in $\mathscr{H}$. For any $\lambda>0$ let $P_{\lambda}$ denote the canonical orthogonal projection onto the finite dimensional subspace

$$
\mathscr{P}_{\lambda}=\operatorname{span}\left\{e_{m}: \lambda_{m} \leq \lambda\right\}
$$

of $\mathscr{H}$, and let $Q_{\lambda}=I-P_{\lambda}$ denote the complementary projection onto the subspace

$$
\mathscr{Q}_{\lambda}=\mathscr{P}_{\lambda}^{\perp}=\mathrm{cl} \operatorname{span}\left\{e_{m}: \lambda_{m}>\lambda\right\}
$$

If $\lambda$ and $m$ satisfy

$$
\lambda_{m} \leq \lambda<\lambda_{m+1}
$$

one has the spectral property:

$$
\begin{gathered}
\operatorname{spec}\left(-\Delta \mid \mathscr{P}_{\lambda}\right)=\left\{\lambda_{1}, \lambda_{2}, \ldots, \lambda_{m}\right\} \subset[0, \lambda], \\
\operatorname{spec}\left(-\Delta \mid \mathscr{Q}_{\lambda}\right)=\left\{\lambda_{m+1}, \lambda_{m+2}, \lambda_{m+3}, \ldots\right\} \subset(\lambda, \infty)
\end{gathered}
$$

for the spectrum of $-\Delta$ restricted to these invariant subspaces.

3.3. The modified equations. Fixing $\lambda>0$ and applying the projections $P_{\lambda}$ and $Q_{\lambda}$ to equation (3.10) we obtain the system

$$
\begin{aligned}
& p^{\prime}=\Delta p+P_{\lambda} \tilde{f}(p, q), \\
& q^{\prime}=\Delta q+Q_{\lambda} \tilde{f}(p, q)
\end{aligned}
$$

where $p=P_{\lambda} u \in \mathscr{P}_{\lambda}$ and $q=Q_{\lambda} u \in \mathscr{Q}_{\lambda}$. One would like to apply the Invariant Manifold Theorem directly to the system (3.14). Unfortunately this cannot be done until some technical modifications of these equations are made. These modifications involve cutting off the nonlinearity outside a certain bounded region in $\mathscr{H}$ in such a way that the Dissipative Condition (II) holds. In local problems, such as the construction of a center manifold, such modifications are routine. However the global problem addressed here is somewhat more delicate. In particular the cut-off functions must be constructed with enough care that the Cone Conditions (V) or (Va) can be verified. The need to verify the Cone Condition introduces a technical problem which did not arise in Foias, Sell, and Temam (1986), and as a result the modification we describe below differs from that used in other theories of inertial manifolds.

The modified equations, to which the Invariant Manifold Theorem will be applied, are

$$
\begin{aligned}
& p^{\prime}=-\phi\left(\|A p\|^{2}\right) A p+\psi\left(\|p\|^{2}\right)\left[p+P_{\lambda} \tilde{f}(p, q)\right] \\
& q^{\prime}=-A q+q+\psi\left(\|p\|^{2}\right) Q_{\lambda} \tilde{f}(p, q)
\end{aligned}
$$

where $A$ is the positive selfadjoint operator

$$
A=I-\triangle \text {, }
$$


$\phi, \psi:[0, \infty) \rightarrow[0,1]$ are $C^{1}$ functions with prescribed properties, and where $\lambda>0$ is appropriately chosen. ${ }^{9}$ The functions $\phi$ and $\psi$ will be fixed in advance, then $\lambda$ will be sought. In particular, $\phi$ and $\psi$ will not depend on $\lambda$. This will enable us to obtain estimates which are independent of $\operatorname{dim} \mathscr{P}_{\lambda}$. In this section we shall show that with $\phi$ and $\psi$ fixed, conditions (I)-(IV) will hold for all sufficiently large $\lambda$. In $\S \S 4$ and 5 we show that for the domains (3.3) and (3.4), there exist arbitrarily large $\lambda$ such that the Uniform Cone Condition (Va) holds.

One property that $\phi$ and $\psi$ should possess is that

$$
\phi(t)=\psi(t)=1 \quad \text { in }\left[0, R^{2}\right]
$$

where $R>0$ is large enough that the universal attractor $\mathfrak{A}$ of $(3.14)$ is contained in the region where the two systems (3.14) and (3.15) agree, that is

$$
\mathfrak{A} \subset\{u \in \mathscr{D}:\|A u\|<R\} .
$$

(Note that $\|u\| \leq\|A u\|$ for $u \in \mathscr{D}$, so that $\|A u\| \leq R$ implies that both $\|A p\| \leq$ $R$ and $\|p\| \leq R$.) We do not address the question of when an attractor $\mathfrak{A}$ exists and satisfies a regularity property such as (3.17). This is a fairly standard matter, and rather than consider it here we shall simply assume that $R$ is a given constant, and we will construct appropriate $\phi$ and $\psi$ satisfying (3.16); see Figure 3. Typically $R$ will be chosen so that $\|A u\|<R$ whenever $u \in \mathfrak{A}$.

With $R>0$ now fixed the precise properties required of $\phi$ and $\psi$ are:

$$
\begin{cases}\phi, \psi:[0, \infty) \rightarrow[0,1] & \text { are } C^{1}, \\ \phi^{\prime}(\tau) \leq 0 & \text { in }[0, \infty), \\ \tau \phi^{\prime}(\tau)+\phi(\tau) \geq 0 & \text { in }[0, \infty), \\ \phi(\tau)=1 & \text { in }\left[0, R^{2}\right], \\ \phi(\tau)=1 / 2 & \text { in }\left[K_{4} R^{2}, \infty\right) \text { for some } K_{4}>1, \\ \psi(\tau)=1 & \text { in }\left[0, K_{4} R^{2}\right], \\ \psi(\tau)=0 & \text { in }\left[K_{5} R^{2}, \infty\right) \text { for some } K_{5}>K_{4} .\end{cases}
$$

These functions equal unity near the origin, and $\psi$ has compact support. The existence of $\psi$ is standard, but constructing $\phi$ is a little delicate. A specific choice of $\phi$, with $K_{4}=5$, is the cubic spline:

$$
\phi(\tau)= \begin{cases}1 & \text { in }\left[0, R^{2}\right], \\ \frac{1}{64}\left[\left(\tau R^{-2}\right)^{3}-9\left(\tau R^{-2}\right)^{2}+15\left(\tau R^{-2}\right)+57\right] & \text { in }\left[R^{2}, 5 R^{2}\right], \\ \frac{1}{2} & \text { in }\left[5 R^{2}, \infty\right) .\end{cases}
$$

\footnotetext{
${ }^{9}$ Notice that the modified equation (3.15) has one feature which is not encountered in other inertial manifold theories, viz. we introduce a term $\phi$ which modifies the linear part of the equation for $p^{\prime}$.
} 


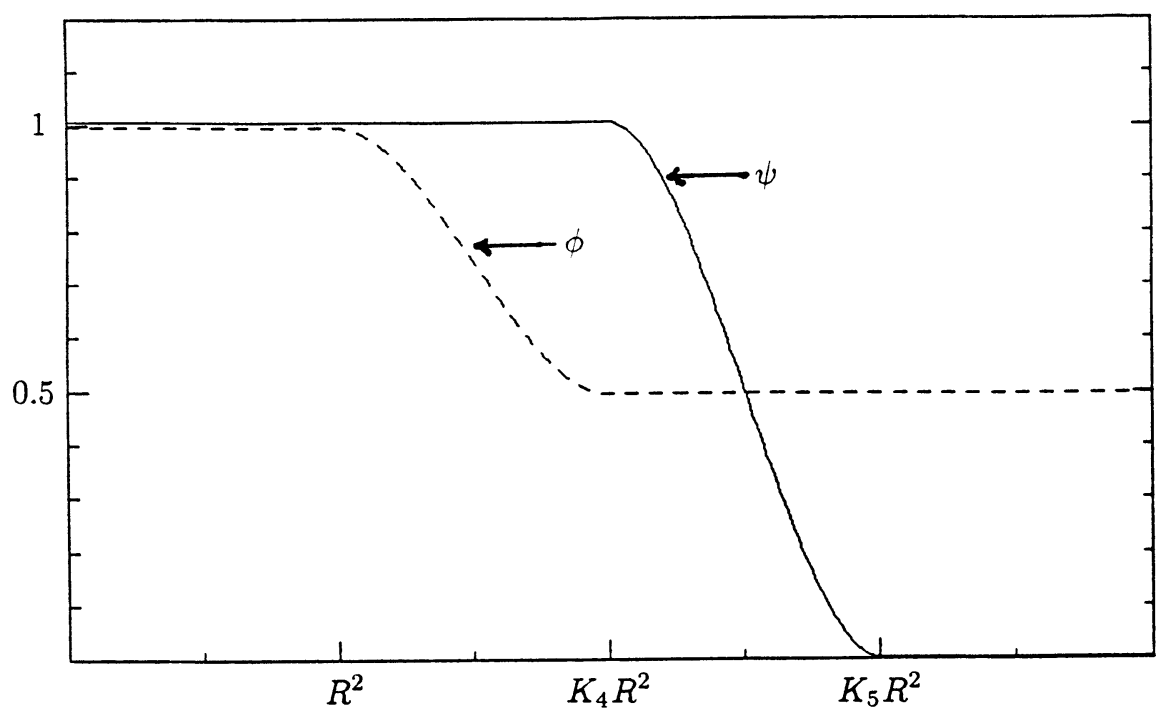

FIGURE 3. The functions $\phi$ and $\psi$.

However, any functions $\phi$ and $\psi$ and quantities $K_{4}$ and $K_{5}$ satisfying the above will do. Assume these have been fixed at this point, and keep them fixed for the remainder of the paper.

For positive quantities $R_{1}$ and $R_{2}$ and for $\lambda>0$ we define

$$
\begin{aligned}
& \mathscr{A}=\left\{p \in \mathscr{P}_{\lambda}:\|p\| \leq R_{1}\right\}, \\
& \mathscr{C}=\left\{q \in \mathscr{D} \subset \mathscr{Q}_{\lambda}:\|A q\| \leq R_{2}\right\}
\end{aligned}
$$

as in $\S 2$. Our primary objective in this section is to show that $R_{1}$ and $R_{2}$ can be chosen so that the following result holds.

Lemma 3.2. Let $\Omega \subset R^{n}$ be a bounded Lipschitz domain with $n=\operatorname{dim} \Omega \leq$ 3 , and let $f$ satisfy (3.6). Fix $\phi$ and $\psi$ satisfying (3.18), and fix boundary conditions (3.5). Then there exist $R_{1}$ and $R_{2}$ such that the hypotheses (I)-(IV) of the Invariant Manifold Theorem A, hold for the system (3.15) for all sufficiently large $\lambda$. Here one has

$$
\begin{aligned}
& A=I-\triangle \\
& F(p, q)=-\phi\left(\|A p\|^{2}\right) A p+\psi\left(\|p\|^{2}\right)\left[p+P_{\lambda} \tilde{f}(p, q)\right], \\
& G(p, q)=q+\psi\left(\|p\|^{2}\right) Q_{\lambda} \tilde{f}(p, q),
\end{aligned}
$$

for the abstract system (2.1).

The next several lemmas will lead to a proof of this result. We assume throughout this section that the hypotheses of Lemma 3.2 hold.

As required by the hypotheses of the Invariant Manifold Theorem, the selfadjoint operator $-A$ generates a strongly continuous semigroup in $\mathscr{Q}_{\lambda}$, and possesses a compact inverse on $\mathscr{Q}_{\lambda}$. Furthermore using Lemma 3.1 one sees 
that both $F$ and $G$ are locally Lipschitz in $\mathscr{H}$. (Note that since $P_{\lambda}$ is a finite dimensional space, the operator $A$ is bounded there. The bound depends on $\lambda$, of course, but that does not matter here.)

3.4. Verification of the Regularity Condition. To prove Lemma 3.2 it is sufficient therefore to find quantities $R_{1}$ and $R_{2}$ so that the conditions (I)-(IV) hold for all sufficiently large $\lambda$. We begin by considering the Regularity Condition (I). The following lemma in fact holds for domains of any dimension $n \geq 1$.

Lemma 3.3. Fix $s>\frac{n}{2}$ and $C>0$. Define

$$
\mathscr{B}=\left\{u \in H^{s}:\|u\|_{H^{s}} \leq C\right\} .
$$

Then for each $\varepsilon>0$ there exists $\delta>0$ such that

$$
\|\tilde{f}(u+h)-\tilde{f}(u)-D \tilde{f}(u) h\| \leq \varepsilon\|h\|
$$

whenever $0<\|h\| \leq \delta$ and both $u, u+h \in \mathscr{B}$. Here $D \tilde{f}(u)$ is the operator on $L^{2}$ defined by

$$
[D \tilde{f}(u) h](x)=D_{u} f(x, u(x)) h(x) .
$$

Furthermore, $D \tilde{f}(u)$ as an element of $\mathscr{L}=\mathscr{L}\left(L^{2}, L^{2}\right)$ varies continuously with $u \in \mathscr{B}$, where $\mathscr{L}$ is endowed with the operator topology inherited from $L^{2}$.

Proof. Fix $\varepsilon>0, r \in\left(\frac{n}{2}, s\right)$, and recall the Sobolev inequalities

$$
\|u\|_{L^{\infty}} \leq C_{1}\|u\|_{H^{r}} \quad \text { and } \quad\|u\|_{L^{\infty}} \leq C_{2}\|u\|_{H^{s}},
$$

for some positive constants $C_{1}$ and $C_{2}$. Also recall the interpolation inequality

$$
\|u\|_{H^{r}} \leq C_{3}\|u\|_{H^{s}}^{r / s}\|u\|^{1-r / s} \text {. }
$$

For $u, u+h \in \mathscr{B}$ we then obtain the inequalities

$$
\begin{gathered}
\|u\|_{L^{\infty}} \leq C_{2} C \\
\|h\|_{L^{\infty}} \leq C_{1}\|h\|_{H^{r}} \leq C_{1} C_{3}\|h\|_{H^{r}}^{r / s}\|h\|^{1-r / s} \leq C_{1} C_{3}(2 C)^{r / s} \delta^{1-r / s}
\end{gathered}
$$

where $\delta$ is to be determined.

By the uniform continuity of $D_{u} f$ there exists $\delta_{0}$ such that

$$
\left|D_{u} f(x, \mu+\eta)-D_{u} f(x, \mu)\right| \leq \varepsilon
$$

whenever $\mu, \eta \in R$ satisfy $|\mu| \leq C_{2} C$ and $|\eta| \leq \delta_{0}$. Upon writing

$$
\begin{aligned}
f(x, u(x)+h(x)) & -f(x, u(x))-D_{u} f(x, u(x)) h(x) \\
& =\int_{0}^{1}\left[D_{u} f(x, u(x)+\theta h(x))-D_{u} f(x, u(x))\right] d \theta h(x),
\end{aligned}
$$

10 The books by Adams (1975) and Edmunds and Evans (1987) are good references for the Sobolev inequalities and the imbedding and trace theorems needed in this paper. 
one sees that the required estimate (3.19) on the difference quotient follows from (3.20-22) whenever $\delta$ is small enough so that $C_{1} C_{3}(2 C)^{r / s} \delta^{1-r / s} \leq \delta_{0}$.

With the same $\delta$ and $\varepsilon$ one sees that if $u_{1}, u_{2} \in \mathscr{B}$ satisfy $\left\|u_{1}-u_{2}\right\| \leq \delta$, then one has $\left\|u_{1}-u_{2}\right\|_{L^{\infty}} \leq \delta_{0}$ and, from (3.22),

$$
\left|D_{u} f\left(x, u_{1}(x)\right)-D_{u} f\left(x, u_{2}(x)\right)\right| \leq \varepsilon \quad \text { in } \Omega .
$$

This implies that $\left\|D \tilde{f}\left(u_{1}\right)-D \tilde{f}\left(u_{2}\right)\right\|_{\text {op }} \leq \varepsilon$, completing the proof.

We note that the operator $D \tilde{f}(u)$ above is not a true Fréchet derivative as the ball $\mathscr{B}$ in $H^{s}$ has empty interior in $L^{2}$.

Lemma 3.4. For any $\lambda, R_{1}$, and $R_{2}$, the Regularity Condition (I) holds for (3.15).

Proof. Noting the boundedness of $A$ on the finite dimensional space $\mathscr{P}_{\lambda}$, one need only check that for any $R_{1}$ and $R_{2}, \tilde{f}$ is $C^{1}$ in the convex set $\mathscr{A} \times \mathscr{C}$. But this is clear from Lemma 3.3 with $s=2$. Indeed for any $\lambda, R_{1}$ and $R_{2}$, there exists $C$ such that

$$
\mathscr{A} \times \mathscr{C} \subset \mathscr{B}=\left\{u \in H^{2}:\|u\|_{H^{2}} \leq C\right\},
$$

because $A^{-1}$ is a bounded linear transformation from $L^{2}$ into $H^{2}$. As $\tilde{f}$ is $C^{1}$ in $\mathscr{B}$, the result follows.

3.5. Verification of the Dissipative Condition. One easily calculates the derivatives $D F(p, q)$ and $D G(p, q)$ acting on an arbitrary $(\rho, \sigma) \in \mathscr{P}_{\lambda} \times \mathscr{Q}_{\lambda}=\mathscr{H}$ :

$$
\begin{aligned}
D F(p, q)(\rho, \sigma)= & -2 \phi^{\prime}\left(\|A p\|^{2}\right)\langle A p, A \rho\rangle A p-\phi\left(\|A p\|^{2}\right) A \rho \\
& +2 \psi^{\prime}\left(\|p\|^{2}\right)\langle p, \rho\rangle\left[p+P_{\lambda} \tilde{f}(p, q)\right] \\
& +\psi\left(\|p\|^{2}\right)\left[\rho+P_{\lambda} D \tilde{f}(p, q)(\rho, \sigma)\right],
\end{aligned}
$$

$D G(p, q)(\rho, \sigma)=\sigma+2 \psi^{\prime}\left(\|p\|^{2}\right)\langle p, \rho\rangle Q_{\lambda} \tilde{f}(p, q)+\psi\left(\|p\|^{2}\right) Q_{\lambda} D \tilde{f}(p, q)(\rho, \sigma)$.

Lemma 3.5. If $R_{1} \geq K_{5}^{1 / 2} R$ then the Dissipative Condition (II) holds.

Proof. With $p \in \operatorname{cl}\left(\mathscr{P}_{\lambda} \backslash \mathscr{A}\right)$, that is, with $\|p\| \geq R_{1} \geq K_{5}^{1 / 2} R$, one has $\psi\left(\|p\|^{2}\right)=0$. Therefore $F(p, 0)=-\phi\left(\|A p\|^{2}\right) A p$, hence

$$
\langle p, F(p, 0)\rangle \leq-\frac{1}{2}\langle p, A p\rangle<0 .
$$

One also sees that $G(p, 0)=0$ for $\|p\| \geq R_{1}$.

3.6. Verification of the Sobolev Condition. We will use the following estimate.

Lemma 3.6. For any $\lambda>0$ and $t>0$ one has

$$
\begin{aligned}
\left\|e^{-A t} Q_{\lambda}\right\|_{\mathrm{op}} & \leq e^{-(1+\lambda) t} \\
\left\|A^{1 / 2} e^{-A t} Q_{\lambda}\right\|_{\mathrm{op}} & \leq(1+\lambda)^{1 / 2} b((1+\lambda) t) \leq(2 e t)^{-1 / 2},
\end{aligned}
$$


where

$$
b(t)= \begin{cases}(2 e t)^{-1 / 2} & \text { in }(0,0.5], \\ e^{-t} & \text { in }[0.5, \infty),\end{cases}
$$

and for any $\theta>0$ one has

$$
\int_{0}^{\infty} \theta^{1 / 2} b(\theta t) d t=2(\theta e)^{-1 / 2} .
$$

Proof. From (3.13), the spectrum of the selfadjoint operator $A=I-\triangle$ restricted to $\mathscr{Q}_{\lambda}=$ range $Q_{\lambda}$ is contained in $(1+\lambda, \infty)$, so the first part of $(3.25)$ is clear. One also has

$$
\left\|A^{1 / 2} e^{-A t} Q_{\lambda}\right\|_{\mathrm{op}} \leq \sup _{\theta \geq 1+\lambda} \theta^{1 / 2} e^{-\theta t}
$$

and a simple argument from the calculus shows the right-hand side of this inequality equals $(1+\lambda)^{1 / 2} b((1+\lambda) t)$. The remainder of the proof is elementary.

Let us recall some properties of the fractional powers $A^{s}, 0 \leq s \leq 1$, of the positive operator $A=I-\triangle$. Let $\mathscr{D}_{s}$ denote the domain of $A^{s}$, with $\mathscr{D}=\mathscr{D}_{1}$ as in (3.9). One then has

$$
\mathscr{D}_{s} \subset H^{2 s} \text {. }
$$

That is, $D_{s}$ consists of those $u \in H^{2 s}$ satisfying the chosen boundary conditions. With Dirichlet boundary conditions and with $s>1 / 4$ any $u \in H^{2 s}$ yields a boundary value $u \in H^{2 s-1 / 2}(\partial \Omega)$ by the Trace Theorem. (For $0<$ $s \leq 1 / 4$ the restriction of $u$ to $\partial \Omega$ is undefined.) With Neumann boundary conditions and with $s>3 / 4$ any $u \in H^{2 s}$ the normal derivative function $\partial u / \partial n \in H^{2 s-3 / 2}(\partial \Omega)$ is well defined. For $0<s \leq 3 / 4, \partial u / \partial n$ is undefined. With periodic boundary conditions the spaces $H^{2 s}$, by definition, consist of functions satisfying the periodic boundary conditions. Indeed, one has

$$
\mathscr{D}_{s}=H^{2 s} \quad \text { if } \begin{cases}0<s \leq 1 / 4 & \text { (Dirichlet) }, \\ 0<s \leq 3 / 4 & \text { (Neumann), } \\ 0<s \leq 1 & \text { (periodic) }\end{cases}
$$

while $D_{s} \subset H^{2 s}$ for other values of $s$ in the range $(0,1]$. In any case, $\mathscr{D}_{s}$ is a closed subspace of $H^{2 s}$, and the quantity $\left\|A^{s} u\right\|$ is a norm on $\mathscr{D}_{s}$ which is equivalent to the norm of $H^{2 s}$.

In the following lemma and subsequent results we shall introduce positive constants $K_{j}$ as needed. As with $K_{1}, \ldots, K_{5}$ already introduced, these constants will not depend on the quantity $\lambda$, for sufficiently large $\lambda$.

Lemma 3.7. Define

$$
\tilde{f}_{0}(u)= \begin{cases}\tilde{f}(u) & (\text { Neumann or periodic }) \\ \tilde{f}(u)-\tilde{f}(0) & \text { (Dirichlet) }\end{cases}
$$


according to the boundary conditions being considered, and let $\mathscr{D}_{1 / 2} \subset \mathscr{H}$ denote the domain of the operator $A^{1 / 2}$. Then

$$
\tilde{f}_{0}(u) \in \mathscr{D}_{1 / 2} \quad \text { whenever } u \in \mathscr{D}_{1 / 2} .
$$

Furthermore there exist $K_{6}$ and $K_{7}$ such that

$$
\left\|A^{1 / 2} \tilde{f}_{0}(u)\right\| \leq K_{6}\left\|A^{1 / 2} u\right\|+K_{7}
$$

for all $u \in \mathscr{D}_{1 / 2}$.

Proof. Consider first the Neumann or periodic boundary conditions, where $\mathscr{D}_{1 / 2}=H^{1}$. For $u \in H^{1}$ we will show that $\tilde{f}_{0}(u) \in H^{1}$ and estimate $\left\|\tilde{f}_{0}(u)\right\|_{H^{1}}$. As

$$
D_{x}[f(x, u(x))]=\left(D_{x} f\right)(x, u(x))+\left(D_{u} f\right)(x, u(x)) D u(x)
$$

the following estimate for the $L^{2}$ norm of this function follows from (3.6):

$$
\left\|D_{x}[f(., u(.))]\right\| \leq K_{1}\|u\|+K_{3}+K_{1}\|D u\|
$$

where $K_{3}=(\operatorname{vol} \Omega)^{1 / 2} K_{2}$. This estimate, combined with Lemma 3.1 and the fact that $\left\|A^{1 / 2} u\right\|$ is an equivalent norm on $H^{1}$, yields the results (3.28), (3.29).

For Dirichlet boundary conditions the estimates are basically the same. One does note however that while $\mathscr{D}_{1 / 2} \subset H^{1}$, (3.28) still holds because

$$
\left[\tilde{f}_{0}(u)\right](x)=f(x, u(x))-f(x, 0)=0
$$

for $x \in \partial \Omega$, since $u(x)=0$ there.

Lemma 3.8. Given any $R_{1}$ there exists $R_{2}$ such that the Sobolev Condition(III) holds for all sufficiently large $\lambda$.

Proof. Beginning with an initial condition $p_{0} \in \mathscr{A}$ (that is, $\left\|p_{0}\right\| \leq R_{1}$ and $\left.q_{0}=0\right)$ we require a uniform estimate $\|A q(t)\| \leq R_{2}$ in any interval $\left[0, t_{0}\right]$ in which $p(t) \in \mathscr{A}$. (Here, of course, $p(t)=p\left(t, p_{0}, 0\right)$ and $q(t)=q\left(t, p_{0}, 0\right)$ are solutions of the initial value problem through $\left(p_{0}, 0\right)$.) By a uniform estimate we mean one which is independent of $t$ and large $\lambda$. We shall use a boot-strap argument and successively obtain estimates of $\|q(t)\|,\left\|A^{1 / 2} q(t)\right\|$, $\left\|A^{1 / 2} p(t)\right\|$, and finally $\|A q(t)\|$, all the time assuming

$$
\|p(t)\| \leq R_{1} \quad \text { in }\left[0, t_{0}\right] .
$$

The constants $K_{j}$ appearing in these estimates may depend on $R_{1}$, but not on $t$ or large $\lambda$.

With constants $K_{1}, K_{3}, K_{6}$, and $K_{7}$ as in (3.7) and (3.29), we fix a quantity $\mu$ satisfying $\mu>1+2 K_{6}$ and consider only values of $\lambda$ satisfying $\lambda>$ $\max \left\{1+K_{1}, \mu\right\}$. We first estimate $\|q(t)\|$. Since $q(0)=0$, one has from the variation of constants formula that

$$
q(t)=\int_{0}^{t} e^{-A(t-s)} Q_{\lambda}\left[q(s)+\tilde{\psi}(s) Q_{\lambda} \tilde{f}(p(s), q(s))\right] d s
$$


where $\tilde{\psi}(s)=\psi\left(\|p(s)\|^{2}\right)$ takes values in $[0,1]$. Therefore

$$
\|q(t)\| \leq \int_{0}^{t} e^{-(1+\lambda)(t-s)}\left[K_{1} R_{1}+\left(1+K_{1}\right)\|q(s)\|+K_{3}\right] d s
$$

by Lemmas 3.1 and 3.6. An application of Gronwall's inequality now yields

$$
\|q(t)\| \leq\left(K_{1} R_{1}+K_{3}\right)\left(\lambda-K_{1}\right)^{-1} .
$$

Now applying $A^{1 / 2}$ to the formula (3.31) and again using Lemmas 3.1 and 3.6, one obtains

$$
\left\|A^{1 / 2} q(t)\right\| \leq \int_{0}^{t}(1+\lambda)^{1 / 2} b((1+\lambda)(t-s))\left[K_{1} R_{1}+\left(1+K_{1}\right)\|q(s)\|+K_{3}\right] d s .
$$

Inserting the estimate (3.32) into the integral above, and using (3.26), one obtains after some manipulation

$$
\left\|A^{1 / 2} q(t)\right\| \leq 2(1+\lambda)^{1 / 2} e^{-1 / 2}\left(K_{1} R_{1}+K_{3}\right)\left(\lambda-K_{1}\right)^{-1} \leq K_{8} \lambda^{-1 / 2}
$$

for some $K_{8}$.

To estimate $\left\|A^{1 / 2} p(t)\right\|$ we first make the decomposition $p(t)=p_{1}(t)+p_{2}(t)$ where

$$
p_{1}(t)=P_{\mu} p(t), \quad p_{2}(t)=\left(I-P_{\mu}\right) p(t)=\left(P_{\lambda}-P_{\mu}\right) u(t),
$$

and then estimate $\left\|A^{1 / 2} p_{1}(t)\right\|$ and $\left\|A^{1 / 2} p_{2}(t)\right\|$ separately. One sees immediately that

$$
\left\|A^{1 / 2} p_{1}(t)\right\| \leq(1+\mu)^{1 / 2} R_{1}
$$

from the spectral properties (3.12) of $A=I-\triangle$ and (3.30). The variation of constants formula for the $p_{2}$-equation yields an estimate of $\left\|A^{1 / 2} p_{2}(t)\right\|$. Indeed one has

$$
p_{2}(t)=e^{-A \eta(t, 0)} p_{2}(0)+\int_{0}^{t} e^{-A \eta(t, s)} \tilde{\psi}(s)\left[p_{2}(s)+\left(P_{\lambda}-P_{\mu}\right) \tilde{f}(p(s), q(s))\right] d s
$$

where $\eta(t, s)=\int_{s}^{t} \tilde{\phi}(r) d r$ and $\tilde{\phi}(r)=\phi\left(\|A p(r)\|^{2}\right)$. As $\tilde{\phi}(r)$ takes values in $\left[\frac{1}{2}, 1\right]$ it follows that

$$
\frac{1}{2}(t-s) \leq \eta(t, s) \leq t-s
$$

when $0 \leq s \leq t$.

In the case of Neumann or periodic boundary conditions Lemma 3.7 implies that

$$
\tilde{f}(p(s), q(s))=\tilde{f}_{0}(p(s), q(s)) \in \mathscr{D}_{1 / 2}
$$

for $s>0$, and so

$$
\begin{aligned}
A^{1 / 2} p_{2}(t)= & A^{1 / 2} e^{-A \eta(t, 0)} p_{2}(0) \\
& +\int_{0}^{t} e^{-A \eta(t, s)} \tilde{\psi}(s)\left[A^{1 / 2} p_{2}(s)+\left(P_{\lambda}-P_{\mu}\right) A^{1 / 2} \tilde{f}_{0}(p(s), q(s))\right] d s
\end{aligned}
$$


Notice that the $A^{1 / 2}$ in this formula can be replaced by $A^{1 / 2} Q_{\mu}$. Taking norms in (3.35) and using Lemmas 3.6 and 3.7 and the estimates (3.33) and (3.34) already obtained, gives

$$
\begin{aligned}
& \left\|A^{1 / 2} p_{2}(t)\right\| \leq(1+\mu)^{1 / 2} b((1+\mu) t / 2) R_{1} \\
& +\int_{0}^{t} e^{-(1+\mu)(t-s) / 2}\left[K_{6}(1+\mu)^{1 / 2} R_{1}+\left(1+K_{6}\right)\left\|A^{1 / 2} p_{2}(s)\right\|+K_{6} K_{8} \lambda^{-1 / 2}+K_{7}\right] d s
\end{aligned}
$$

and again applying Gronwall's inequality yields

$$
\begin{aligned}
\left\|A^{1 / 2} p_{2}(t)\right\| \leq & (1+\mu)^{1 / 2} b((1+\mu) t / 2) R_{1} \\
& +\left(1+K_{6}\right)(1+\mu)^{1 / 2} R_{1} \int_{0}^{t} e^{-\left(\mu-1-2 K_{6}\right)(t-s) / 2} b((1+\mu) s / 2) d s \\
& +2\left[K_{6}(1+\mu)^{1 / 2} R_{1}+K_{6} K_{8} \lambda^{-1 / 2}+K_{7}\right]\left[\mu-1-2 K_{6}\right]^{-1} .
\end{aligned}
$$

One sees from this and from the formula for $b$ in Lemma 3.6 that

$$
\left\|A^{1 / 2} p_{2}(t)\right\| \leq K_{9} t^{-1 / 2}+K_{10} .
$$

In the case of Dirichlet boundary conditions an estimate of the same form (3.36) is also obtained. An additional term

$$
\int_{0}^{t} A^{1 / 2} e^{-A \eta(t, s)} \tilde{\psi}(s)\left(P_{\lambda}-P_{\mu}\right) \tilde{f}(0) d s
$$

appears in the right-hand side of (3.28), and one sees the norm of this term is bounded by a constant. Indeed from (3.7) and (3.26) one obtains

$$
\begin{aligned}
\left\|\int_{0}^{t} A^{1 / 2} e^{-A \eta(t, s)} \tilde{\psi}(s)\left(P_{\lambda}-P_{\mu}\right) \tilde{f}(0) d s\right\| \\
\quad \leq\left[\int_{0}^{t}(1+\mu)^{1 / 2} b((1+\mu)(t-s) / 2) d s\right]\|\tilde{f}(0)\| \\
\quad \leq 2^{3 / 2}(1+\mu)^{-1 / 2} e^{-1 / 2} K_{3} .
\end{aligned}
$$

The final step in the proof of this lemma is to estimate $\|A q(t)\|$. With Neumann or periodic boundary conditions one has from (3.31)

Thus with

$$
A q(t)=\int_{0}^{t} A^{1 / 2} e^{-A(t-s)} A^{1 / 2}\left[q(s)+\tilde{\psi}(s) Q_{\lambda} \tilde{f}_{0}(p(s), q(s)] d s .\right.
$$

$$
\gamma(s)=K_{6}(1+\mu)^{1 / 2} R_{1}+K_{6}\left(K_{9} s^{-1 / 2}+K_{10}\right)+\left(1+K_{6}\right) K_{8} \lambda^{-1 / 2}+K_{7},
$$

it follows from Lemma 3.7, (3.33), (3.34), and (3.36) that

$$
\begin{aligned}
\|A q(t)\| & \leq \int_{0}^{t}(1+\lambda)^{1 / 2} b((1+\lambda)(t-s)) \gamma(s) d s \\
& \leq K_{11} \lambda^{-1 / 2}+\int_{0}^{t}(1+\lambda)^{1 / 2} b((1+\lambda)(t-s)) K_{6} K_{9} s^{-1 / 2} d s \\
& \leq K_{11} \lambda^{-1 / 2}+\int_{0}^{t}(2 e)^{-1 / 2} K_{6} K_{9}(t-s)^{-1 / 2} s^{-1 / 2} d s \\
& =K_{11} \lambda^{-1 / 2}+(2 e)^{-1 / 2} K_{6} K_{9} \pi .
\end{aligned}
$$


Thus one has the estimate $\|A q(t)\| \leq R_{2}$ for all sufficiently large $\lambda$, provided $R_{2}>(2 e)^{-1 / 2} K_{6} K_{9} \pi$. In the case of the Dirichlet conditions an additional term appears, as above, but the final result still holds.

\subsection{Verification of the Linear Stability Condition.}

Lemma 3.9. For any fixed $R_{1}$ and $R_{2}$, the Linear Stability Condition (IV) holds for all sufficiently large $\lambda$.

Proof. Given $R_{1}$ and $R_{2}$, one has for any $u=(p, q) \in \mathscr{A} \times \mathscr{C}$ that

$$
\|u\| \leq\|p\|+\|q\| \leq\|p\|+\|A q\| \leq R_{1}+R_{2}
$$

and hence

$$
\|\tilde{f}(u)\| \leq K_{1}\left(R_{1}+R_{2}\right)+K_{3}
$$

by Lemma 3.1. One also has $\|D \tilde{f}(u)\|_{\mathrm{op}} \leq K_{1}$ by (3.6). Estimating in (3.24) thus gives a bound

$$
\|D G(p, q)\|_{\mathrm{op}} \leq 1+2\left(\sup \left|\psi^{\prime}\right|\right) R_{1}\left[K_{1}\left(R_{1}+R_{2}\right)+K_{3}\right]+K_{1}
$$

independent of $\lambda$. As

$$
-\langle q, A q\rangle \leq-(1+\lambda)\|q\|^{2}
$$

holds for $q \in \mathscr{Q}_{\lambda} \cap \mathscr{D}$, it suffices to take

$$
\lambda>1+4\left(\sup \left|\psi^{\prime}\right|\right) R_{1}\left[K_{1}\left(R_{1}+R_{2}\right)+K_{3}\right]+2 K_{1}
$$

to satisfy the Linear Stability Condition.

3.8. Partial verification of the Uniform Cone Condition. We complete this section by making a partial verification of the Uniform Cone Condition (Va), valid for the general two- or three-dimensional domains $\Omega$ considered here. In particular we will show that the Uniform Cone Condition is satisfied when $\|A p\| \geq K_{4}^{1 / 2} R$. The full Uniform Cone Condition will be proved (in $\S \S 4$ and 5) only for the special rectangular and cubic domains $\Omega_{2}$ and $\Omega_{3}$.

Lemma 3.10. Let $v, w$, and $y$ be elements of a real Hilbert space. Then

$$
-\|y\|^{2}(\|v\|\|w\|-\langle v, w\rangle) \leq 2\langle v, y\rangle\langle w, y\rangle \leq\|y\|^{2}(\|v\|\|w\|+\langle v, w\rangle) .
$$

Proof. Without loss of generality we can assume that $y \in \operatorname{span}\{v, w\}$. (For the general $y$ one may write $y=y_{1}+y_{2}$ where $y_{1} \in \operatorname{span}\{v, w\}$ and $\left\langle v, y_{2}\right\rangle=$ $\left\langle w, y_{2}\right\rangle=0$. In this case one sees that if the inequality (3.37) holds for $y_{1}$ then it also holds for $y$.) Also, without loss of generality one may assume that $\|v\|=\|w\|=\|y\|=1$, as the expressions in (3.37) all are homogeneous of the same degree. Finally, by choosing appropriate coordinates in $\operatorname{span}\{v, w\}$, which we assume, without loss of generality, to be two-dimensional, one may assume $v$ and $w$ are symmetric about the horizontal axis:

$$
v=(\cos \alpha, \sin \alpha), \quad w=(\cos \alpha,-\sin \alpha)
$$


for some $\alpha$. Writing the unit vector $y$ as $y=(\cos \beta, \sin \beta)$, one sees that (3.37) is equivalent to

$$
-(1-\cos 2 \alpha) \leq \cos 2 \alpha+\cos 2 \beta \leq 1+\cos 2 \alpha
$$

which always holds.

Lemma 3.11. For any $\lambda$ and any $(p, q)$ and $(\rho, \sigma)$ in $\mathscr{P}_{\lambda} \times \mathscr{Q}_{\lambda}$ one has

$$
V^{\prime} \leq-\langle\sigma, A \sigma\rangle+\phi\left(\|A p\|^{2}\right)\|A \rho\|\|\rho\|+W
$$

where

$$
\begin{aligned}
W= & \|\sigma\|^{2}+2 \psi^{\prime}\left(\|p\|^{2}\right)\langle p, \rho\rangle[\langle(-\rho, \sigma), \tilde{f}(p, q)\rangle-\langle\rho, p\rangle] \\
& +\psi\left(\|p\|^{2}\right)\left[\langle(-\rho, \sigma), D \tilde{f}(p, q)(\rho, \sigma)\rangle-\|\rho\|^{2}\right] .
\end{aligned}
$$

Furthermore, if $(p, q) \in \mathscr{A} \times \mathscr{C}$ and $\|\rho\|,\|\sigma\| \leq 1$, then the estimate

$$
|W| \leq K_{12}
$$

holds for some $K_{12}$ depending only on $R_{1}$ and $R_{2}$, but not on $\lambda$.

Proof. From (2.2), (3.23), and (3.24) one has

$$
\begin{aligned}
V^{\prime} & =\left\langle\sigma, \sigma^{\prime}\right\rangle-\left\langle\rho, \rho^{\prime}\right\rangle \\
& =-\langle\sigma, A \sigma\rangle+\langle\sigma, D G(p, q)(\rho, \sigma)\rangle-\langle\rho, D F(p, q)(\rho, \sigma)\rangle \\
& =-\langle\sigma, A \sigma\rangle+2 \phi^{\prime}\left(\|A p\|^{2}\right)\langle A p, A \rho\rangle\langle\rho, A p\rangle+\phi\left(\|A p\|^{2}\right)\langle\rho, A \rho\rangle+W
\end{aligned}
$$

with $W$ as in (3.38). By Lemma 3.10 one has

$$
2\langle A p, A \rho\rangle\langle\rho, A p\rangle \geq-\|A p\|^{2}[\|A \rho\|\|\rho\|-\langle A \rho, \rho\rangle]
$$

and so, as $\phi^{\prime}(\tau) \leq 0$ and $\phi^{\prime}(\tau) \tau+\phi(\tau) \geq 0$ with $\tau=\|A p\|^{2}$, one has

$$
\begin{aligned}
V^{\prime} & \leq-\langle\sigma, A \sigma\rangle-\phi^{\prime}(\tau) \tau[\|A \rho\|\|\rho\|-\langle A \rho, \rho\rangle]+\phi(\tau)\langle\rho, A \rho\rangle+W \\
& =-\langle\sigma, A \sigma\rangle-\phi^{\prime}(\tau) \tau\|A \rho\|\|\rho\|+\left[\phi^{\prime}(\tau) \tau+\phi(\tau)\right]\langle A \rho, \rho\rangle+W \\
& \leq-\langle\sigma, A \sigma\rangle-\phi^{\prime}(\tau) \tau\|A \rho\|\|\rho\|+\left[\phi^{\prime}(\tau) \tau+\phi(\tau)\right]\|A \rho\|\|\rho\|+W \\
& =-\langle\sigma, A \sigma\rangle+\phi(\tau)\|A \rho\|\|\rho\|+W
\end{aligned}
$$

as claimed.

The bound (3.39) for $W$ follows from the bound in Lemma 3.1 for $\|\tilde{f}(p, q)\|$, and from the bounds $\|D \tilde{f}(p, q)\|_{\mathrm{op}} \leq K_{1},\|p\| \leq R_{1}$, and $\|q\| \leq$ $\|A q\| \leq R_{2}$ in $\mathscr{A} \times \mathscr{C}$.

Lemma 3.12. Given $R_{1}$ and $R_{2}$, then for all sufficiently large $\lambda$ the inequality $V^{\prime} \leq-\xi$ of the Uniform Cone Condition (Va) holds for any $(p, q) \in \mathscr{A} \times \mathscr{C}$ and $(\rho, \sigma) \in \mathscr{P}_{\lambda} \times \mathscr{Q}_{\lambda}$ with $\|\rho\|=\|\sigma\|=1$, provided

$$
\|A p\| \geq K_{4}^{1 / 2} R
$$

also holds. 
Proof. Now (3.40) implies that $\phi\left(\|A p\|^{2}\right)=1 / 2$, so by Lemma 3.11 one has

$$
\begin{aligned}
V^{\prime} & \leq-\langle\sigma, A \sigma\rangle+\frac{1}{2}\|A \rho\|+K_{12} \\
& \leq-\left(1+\lambda_{m+1}\right)+\frac{1}{2}\left(1+\lambda_{m}\right)+K_{12} \\
& \leq-\frac{1}{2}\left(1+\lambda_{m+1}\right)+K_{12}
\end{aligned}
$$

where $\lambda_{m} \leq \lambda<\lambda_{m+1}$. As $\lambda \rightarrow \infty$ one has $\lambda_{m+1} \rightarrow \infty$, and the result is proved.

\section{The Principle of Spatial Averaging}

In order to complete the verification of the Uniform Cone Condition, and thereby obtain an invariant manifold, one needs rather detailed information about the asymptotics of both the eigenvalues and eigenfunctions of the Laplacian. Indeed, the estimates on $V^{\prime}$ now become quite delicate, and we are able to obtain the Cone Condition only for very special domains $\Omega$ and carefully chosen values of $\lambda$.

We retain the notation of the previous section, as well as the standing assumptions including $n=\operatorname{dim} \Omega \leq 3$.

Fix $R_{1}$ and $R_{2}$ so that conditions (I)-(IV) all hold, for all sufficiently large $\lambda$. This can be done by applying Lemma 3.2. In view of Lemma 3.12, one also has the Uniform Cone Condition (Va) holding in that part of $\mathscr{A} \times \mathscr{C}$ where $\|A p\| \geq K_{4}^{1 / 2} R$. We therefore wish to consider $(p, q) \in \mathscr{A} \times \mathscr{C}$ where

$$
\|A p\| \leq K_{4}^{1 / 2} R \text {. }
$$

Since $\|p\| \leq\|A p\|$, for such points one has $\psi\left(\|p\|^{2}\right)=1, \psi^{\prime}\left(\|p\|^{2}\right)=0$, and $\phi\left(\|A p\|^{2}\right) \leq 1$. Thus with $\|\rho\|=\|\sigma\|=1$, Lemma 3.11 yields

$$
\begin{aligned}
& V^{\prime} \leq-\langle\sigma, A \sigma\rangle+\|A \rho\|+W, \\
& W=\langle(-\rho, \sigma), D \tilde{f}(p, q)(\rho, \sigma)\rangle .
\end{aligned}
$$

The proof that $V^{\prime} \leq-\xi$, at least for the domains $\Omega$ of interest, involves considering two possibilities: either

(1) $-\langle\sigma, A \sigma\rangle+\|A \rho\|$ is sufficiently negative to overcome the bound $|W| \leq$ $K_{12}$, where $K_{12}$ is given by (3.39); or

(2) $-\langle\sigma, A \sigma\rangle+\|A \rho\|$ is negative and bounded away from zero, while $W$ is close to zero.

Case (1) is related to the occurrence of large gaps in the spectrum of $A=I-\triangle$. As noted in the introduction, if

$$
\limsup _{m \rightarrow \infty}\left(\lambda_{m+1}-\lambda_{m}\right)=\infty
$$

then (1) is easily achieved for appropriate $\lambda$. Such is the case, for example, for a square domain $\Omega_{2}=(0,2 \pi)^{2}$, see Kamaev (1981) and Foias, Sell, and Temam (1986). 
If (4.1) fails, as it does for the cube $\Omega_{3}=(0,2 \pi)^{3}$, we must use (2) to show $V^{\prime} \leq-\xi$. In doing so we introduce a new property, the Principle of Spatial Averaging, which certain domains $\Omega$ may possess.

For any $v \in L_{\infty}$ we let $B_{v}$ denote the operator on $L^{2}$ defined by

$$
\left(B_{v} u\right)(x)=v(x) u(x), \quad u \in L^{2},
$$

and let $\bar{v}$ denote the mean value

$$
\bar{v}=(\operatorname{vol} \Omega)^{-1} \int_{\Omega} v(x) d x .
$$

Definition. For a given (bounded, Lipschitz) domain $\Omega \subset R^{n}, n \leq 3$, and choice of boundary conditions (3.5) for the Laplacian, we say the Principle of Spatial Averaging, or PSA, holds if there exists a quantity $\xi>0$, such that for every $\varepsilon>0$ and $\kappa>0$ there exist arbitrarily large $\lambda>\kappa$, such that

$$
\left\|\left(P_{\lambda+\kappa}-P_{\lambda-\kappa}\right)\left(B_{v}-\bar{v} I\right)\left(P_{\lambda+\kappa}-P_{\lambda-\kappa}\right)\right\|_{\mathrm{op}} \leq \varepsilon\|v\|_{H^{2}}
$$

holds whenever $v \in H^{2}$; and such that

$$
\lambda_{m+1}-\lambda_{m} \geq \xi
$$

where $m$ satisfies $\lambda_{m} \leq \lambda<\lambda_{m+1}$.

Very roughly, the inequality (4.2) means that for functions $u(x)$ whose Fourier decomposition is composed of eigenstates corresponding to spectra in the interval $(\lambda-\kappa, \lambda+\kappa]$, the multiplication operator $B_{v}$ "looks like" a scalar multiple of the identity operator for smooth $v(x)$. One thinks of the rapid spatial oscillation of $u(x)$ as homogenizing, or averaging, the function $v(x)$; hence the term principle of spatial averaging.

The main reason for defining the Principle of Spatial Averaging only for domains in $R^{n}$ with $n \leq 3$ is that we want the multiplication operator $B_{v}$ to be bounded for $v \in H^{2}$. In other words, we need the Sobolev imbedding $H^{2} \subset L^{\infty}$, which is valid for $n \leq 3$. However, even if we were to take a smoother norm $\|v\|_{H^{s}}, s>2$ in (4.2), PSA would still fail in certain domains of dimension $n \geq 4$.

It is not clear at all for which domains and boundary conditions PSA holds; indeed, this issue is very much open. If the spectral gap condition (4.1) is satisfied (as with the square) then PSA trivially holds, since one may choose $\lambda$ so that the interval $(\lambda-\kappa, \lambda+\kappa]$ contains no $\lambda_{m}$ and hence $P_{\lambda+\kappa}-P_{\lambda-\kappa}=0$. On the other hand, for the cubic domain $\Omega_{3}=(0,2 \pi)^{3}$ where (4.1) fails, we shall show that PSA does hold. We also show any rectangular domain ${ }^{12}$

${ }^{11}$ See the Remark following Proposition 5.2.

12 The gap condition (4.1) is satisfied for any rectangular domain $\Omega_{2}$ where the ratio $\left(a_{1} / a_{2}\right)^{2}$ is rational. This is a consequence of the Gap Theorem proved in the Appendix to this paper. It is important to note that PSA holds on $\Omega_{2}$ for any choice of $a_{1}$ and $a_{2}$, provided of course that they are positive. 
$\Omega_{2}=\left(0,2 \pi / a_{1}\right) \times\left(0,2 \pi / a_{2}\right)$ satisfies PSA. Because of Theorem C, which is stated below, it would be nice to know if PSA holds on any other type region in $R^{n}, n \leq 3$.

For a general two-dimensional domain it is known that the asymptotic density of the eigenvalues is linear, i.e., for some $C>0$ one has

$$
\lambda_{m} \sim \mathrm{Cm} \text {. }
$$

Thus at least (4.3) holds for some $\xi$ and for infinitely many $m$. For a general three-dimensional domain even (4.3) is in doubt, as the increasing density of eigenvalues

$$
\lambda_{m} \sim \mathrm{Cm}^{2 / 3}
$$

allows the possibility that $\lim \left(\lambda_{m+1}-\lambda_{m}\right)=0$. (For the cube $\Omega_{3}$ one has however $\lambda_{m+1}-\lambda_{m} \geq 1$ infinitely often, as each eigenvalue is an integer.)

The importance of PSA is that it implies the Cone Condition, and hence the existence of an inertial manifold.

Theorem C. Assume that the domain $\Omega$ and the boundary conditions for $-\triangle$ satisfy PSA. Assume $f$ satisfies the regularity and growth conditions (3.6); assume also the function $D_{u} f$ is $C^{2}$ in $\bar{\Omega} \times R$. Fix functions $\phi$ and $\psi$ satisfying (3.18). Then there exists arbitrarily large $\lambda$ such that the system (3.15) satisfies all the hypotheses, including the Uniform Cone Condition (Va), of the Invariant Manifold Theorems $\mathrm{A}$ and $\mathrm{B}$.

Note that the conclusion of Theorem $C$ holds only for certain large $\lambda$, not necessarily for all large $\lambda$.

Before proving this result we need the following lemma.

Lemma 4.1. Assume $f: \bar{\Omega} \times R \rightarrow R$ is differentiable with respect to $u \in R$, and that $D_{u} f$ is a $C^{2}$ function in $\bar{\Omega} \times R$. Then for any $u \in H^{2}$ the function

$$
v(x)=D_{u} f(x, u(x))
$$

belongs to $H^{2}$. Moreover, given $C_{1}$ there exists $C_{2}$ such that

$$
\|u\|_{H^{2}} \leq C_{1} \Longrightarrow\|v\|_{H^{2}} \leq C_{2} .
$$

Proof. One has

$$
\begin{aligned}
D v(x)= & D_{x u} f(x, u(x))+D_{u u} f(x, u(x)) D u(x), \\
D^{2} v(x)= & D_{x x u} f(x, u(x))+2 D_{x u u} f(x, u(x)) D u(x) \\
& +D_{u u u} f(x, u(x))[D u(x)]^{2}+D_{u u} f(x, u(x)) D^{2} u(x) .
\end{aligned}
$$

With the bound $\|u\|_{H^{2}} \leq C_{1}$ and the continuous embedding $H^{2} \subset L^{\infty}$ (since $n \leq 3)$, one obtains $L^{\infty}$ bounds on the derivatives $D_{\alpha} f(x, u(x))$ occurring above, and these bounds depend only on $C_{1}$. Therefore one only need observe that $D u, D^{2} u$, and $(D u)^{2}$ be in bounded sets in $L^{2}$ to conclude the same for 
$D v$ and $D^{2} v$. The $L^{2}$-norms $\|D u\|$ and $\left\|D^{2} u\right\|$ are bounded by assumption, while the bound for

$$
\left\|(D u)^{2}\right\|=\|D u\|_{L^{4}}^{2}
$$

follows from the embedding $H^{1} \subset L^{4}$.

Proof of Theorem $\mathrm{C}$. Recall that the quantities $R_{1}$ and $R_{2}$ have been fixed such that for all large $\lambda$, all the required conditions hold except the Uniform Cone Condition in the range

$$
\|A p\| \leq K_{4}^{1 / 2} R .
$$

Thus all that remains is to prove that there exist arbitrarily large $\lambda$ such that $V^{\prime} \leq-\xi / 2$ whenever $u=(p, q) \in \mathscr{A} \times \mathscr{C}, p$ satisfies (4.4), and

$$
\|\rho\|=\|\sigma\|=1 .
$$

Observe immediately that by Lemma 4.1, inequality (4.4) and $\|A q\| \leq R_{2}$ (from $q \in \mathscr{C}$ ) imply that the function $v(x)=D_{u} f(x, u(x))$ lies in a bounded set in $H^{2}$, i.e.,

$$
\|v\|_{H^{2}} \leq K_{13} .
$$

Here $K_{13}$ does not depend on $\lambda$. Note also from (3.6) that

$$
W=\left\langle(-\rho, \sigma), B_{v}(\rho, \sigma)\right\rangle, \quad\left\|B_{v}\right\|_{\mathrm{op}} \leq K_{1}, \quad \text { and } \quad|\bar{v}| \leq K_{1} \operatorname{vol} \Omega
$$

in this notation.

With $\xi$ satisfying (4.3), choose quantities $\varepsilon, \kappa$, and $\delta$ such that

$$
\left(2 \delta^{2}+4 \delta+\delta^{2} \operatorname{vol} \Omega\right) K_{1}+2 \varepsilon K_{13}<\xi / 2, \quad \kappa \delta^{2}>2 K_{12}+\xi,
$$

and choose $\lambda>\kappa-1$ sufficiently large that the conclusion (4.2) of PSA holds. (One may also assume $\lambda$ is large enough that all other conditions required by Theorem B hold.) The quantities $\varepsilon, \kappa, \delta$, and $\lambda$ will be kept fixed for the remainder of the proof.

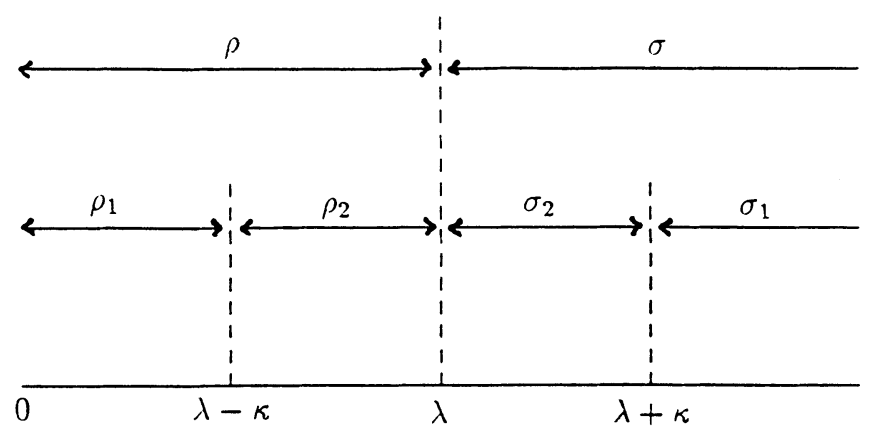

FIGURE 4. Decomposition for $\rho$ and $\sigma$ 
With $v \in H^{2}$ satisfying (4.6), and $(\rho, \sigma) \in \mathscr{P}_{\lambda} \times \mathscr{Q}_{\lambda}$ satisfying (4.5), we must show that $V^{\prime} \leq-\xi / 2$. As shown in Figure 4 we decompose $\rho=\rho_{1}+\rho_{2}$ and $\sigma=\sigma_{1}+\sigma_{2}$ where

$$
\begin{array}{ll}
\rho_{1}=P_{\lambda-\kappa} \rho, & \rho_{2}=\left(I-P_{\lambda-\kappa}\right) \rho=\left(P_{\lambda}-P_{\lambda-\kappa}\right) \rho, \\
\sigma_{1}=Q_{\lambda+\kappa} \sigma, & \sigma_{2}=\left(I-Q_{\lambda+\kappa}\right) \sigma=\left(Q_{\lambda}-Q_{\lambda+\kappa}\right) \sigma .
\end{array}
$$

First suppose $\left\|\rho_{1}\right\| \geq \delta$. Since $\left\langle\rho_{1}, \rho_{2}\right\rangle=0$, one has $\|\rho\|^{2}=\left\|\rho_{1}\right\|^{2}+\left\|\rho_{2}\right\|^{2}$, or $\left\|\rho_{2}\right\|^{2}=1-\left\|\rho_{1}\right\|^{2}$ since $\|\rho\|=1$. Consequently

$$
\begin{aligned}
V^{\prime} & \leq-\langle\sigma, A \sigma\rangle+\|A \rho\|+W \\
& \leq-\left(1+\lambda_{m+1}\right)+\left(\left\|A \rho_{1}\right\|^{2}+\left\|A \rho_{2}\right\|^{2}\right)^{1 / 2}+K_{12} \\
& \leq-\left(1+\lambda_{m+1}\right)+\left[(1+\lambda-\kappa)^{2}\left\|\rho_{1}\right\|^{2}+(1+\lambda)^{2}\left\|\rho_{2}\right\|^{2}\right]^{1 / 2}+K_{12} \\
& =-\left(1+\lambda_{m+1}\right)+\left[(1+\lambda)^{2}-2 \kappa\left(1+\lambda-\frac{\kappa}{2}\right)\left\|\rho_{1}\right\|^{2}\right]^{1 / 2}+K_{12} .
\end{aligned}
$$

By using the inequality $\left[a^{2}-2 b\right]^{1 / 2} \leq a-a^{-1} b$, we next obtain

$$
\begin{aligned}
V^{\prime} & \leq-\left(1+\lambda_{m+1}\right)+(1+\lambda)-\kappa(1+\lambda)^{-1}\left(1+\lambda-\frac{\kappa}{2}\right)\left\|\rho_{1}\right\|^{2}+K_{12} \\
& \leq-\kappa(1+\lambda)^{-1}\left(1+\lambda-\frac{\kappa}{2}\right)\left\|\rho_{1}\right\|^{2}+K_{12} \\
& \leq-\frac{\kappa \delta^{2}}{2}+K_{12}<-\xi / 2 .
\end{aligned}
$$

On the other hand, suppose $\left\|\sigma_{1}\right\| \geq \delta$. As before one has $\left\|\sigma_{2}\right\|^{2}=1-\left\|\sigma_{1}\right\|^{2}$ and consequently

$$
\begin{aligned}
V^{\prime} & \leq-\left\langle\sigma_{1}, A \sigma_{1}\right\rangle-\left\langle\sigma_{2}, A \sigma_{2}\right\rangle+\|A \rho\|+W \\
& \leq-(1+\lambda+\kappa)\left\|\sigma_{1}\right\|^{2}-(1+\lambda)\left\|\sigma_{2}\right\|^{2}+\left(1+\lambda_{m}\right)+K_{12} \\
& =-(1+\lambda+\kappa)\left\|\sigma_{1}\right\|^{2}-(1+\lambda)\left(1-\left\|\sigma_{1}\right\|^{2}\right)+\left(1+\lambda_{m}\right)+K_{12} \\
& =-\kappa\left\|\sigma_{1}\right\|^{2}-\left(\lambda-\lambda_{m}\right)+K_{12} \\
& \leq-\kappa \delta^{2}+K_{12}<-\xi / 2 .
\end{aligned}
$$

Finally, suppose both $\left\|\rho_{1}\right\| \leq \delta$ and $\left\|\sigma_{1}\right\| \leq \delta$. Then

$$
V^{\prime} \leq-\left(1+\lambda_{m+1}\right)+\left(1+\lambda_{m}\right)+W \leq-\xi+W,
$$

and

$$
\begin{aligned}
W & =\left\langle(-\rho, \sigma), B_{v}(\rho, \sigma)\right\rangle=\int_{\Omega} v(-\rho+\sigma)(\rho+\sigma) d x \\
& =-\int_{\Omega} v \rho^{2} d x-\int_{\Omega} v \rho \sigma d x+\int_{\Omega} v \sigma \rho d x+\int_{\Omega} v \sigma^{2} d x \\
& =\int_{\Omega} v\left(\sigma^{2}-\rho^{2}\right) d x .
\end{aligned}
$$


Consequently one has

$$
\begin{aligned}
|W| & =\left|\int_{\Omega} v\left(\left[\sigma_{1}+\sigma_{2}\right]^{2}-\left[\rho_{1}+\rho_{2}\right]^{2}\right) d x\right| \\
& \leq\left|\int_{\Omega} v\left(\sigma_{1}^{2}+2 \sigma_{1} \sigma_{2}-\rho_{1}^{2}-2 \rho_{1} \rho_{2}\right) d x\right|+\left|\int_{\Omega} v\left(\sigma_{2}^{2}-\rho_{2}^{2}\right) d x\right| .
\end{aligned}
$$

By using the Schwarz inequality and (4.7) we get

$$
\begin{aligned}
|W| \leq & \left\|B_{v}\right\|_{\text {op }}\left\{\left\|\sigma_{1}\right\|^{2}+\left\|\rho_{1}\right\|^{2}+2\left(\left\|\sigma_{1}\right\|^{2}+\left\|\rho_{1}\right\|^{2}\right)^{1 / 2}\left(\left\|\sigma_{1}\right\|^{2}+\left\|\rho_{1}\right\|^{2}\right)^{1 / 2}\right\} \\
& +\left|\int_{\Omega} \bar{v}\left(\sigma_{2}^{2}-\rho_{2}^{2}\right) d x\right|+\left|\int_{\Omega}(v-\bar{v})\left(\sigma_{2}^{2}-\rho_{2}^{2}\right) d x\right| \\
\leq & K_{1}\left(2 \delta^{2}+4 \delta\right)+|\bar{v}| \delta^{2}+\left|\left\langle\sigma_{2},\left(B_{v}-\bar{v} I\right) \sigma_{2}\right\rangle\right|+\left|\left\langle\rho_{2},\left(B_{v}-\bar{v} I\right) \rho_{2}\right\rangle\right| \\
\leq & K_{1}\left(2 \delta^{2}+4 \delta+\delta^{2} \operatorname{vol} \Omega\right)+\varepsilon\left(\left\|\sigma_{2}\right\|^{2}+\left\|\rho_{2}\right\|^{2}\right)\|v\|_{H^{2}} \\
\leq & K_{1}\left(2 \delta^{2}+4 \delta+\delta^{2} \operatorname{vol} \Omega\right)+2 \varepsilon K_{13}<\xi / 2,
\end{aligned}
$$

hence $V^{\prime}<-\xi / 2$.

\section{The UNiform CONE CONDITIONS FOR $\Omega_{2}$ AND $\Omega_{3}$}

In this section we verify PSA for the two-dimensional rectangle and threedimensional cubic domains

$$
\begin{aligned}
& \Omega_{2}=\left(0,2 \pi / a_{1}\right) \times\left(0,2 \pi / a_{2}\right), \\
& \Omega_{3}=(0,2 \pi)^{3},
\end{aligned}
$$

with either Dirichlet, Neumann, or periodic boundary conditions. For $\Omega_{2}$ we assume only that $a_{1}$ and $a_{2}$ are positive. We begin by noting that a complete orthonormal set of eigenfunctions of $-\triangle$ for these domains is given by

$$
e_{k}(x)=\left\{\begin{array}{lll}
2^{n / 2}\left(\operatorname{vol} \Omega_{n}\right)^{-1 / 2} \sin a k x, & k \in Z_{+}^{n} & \text { (Dirichlet) } \\
2^{n / 2}\left(\operatorname{vol} \Omega_{n}\right)^{-1 / 2} \cos a k x, & k \in Z_{\oplus}^{n} & \text { (Neumann) } \\
\left(\operatorname{vol} \Omega_{n}\right)^{-1 / 2} e^{i a k x}, & k \in Z^{n} & \text { (periodic) }
\end{array}\right.
$$

for the indicated boundary conditions, with corresponding eigenvalue

$$
\lambda=|a k|^{2} \text {. }
$$

Here we denote

$$
\sin a k x=\Pi_{j=1}^{n} \sin a_{j} k_{j} x_{j}
$$

and likewise for $\cos a k x$ and $e^{i a k x}$, where $a=\left(a_{1}, a_{2}\right)$ in the case of $\Omega_{2}$ and $a=(1,1,1)$ for $\Omega_{3}$. The notation $a k$ refers to the vector

$$
a k=\left(a_{1} k_{1}, \ldots, a_{n} k_{n}\right)
$$

and so the eigenvalue (5.2) is $\lambda=\sum_{j=1}^{n} a_{j}^{2} k_{j}^{2}$. Also, the positive integers are denoted by $Z_{+}$, while $Z_{\oplus}=Z_{+} \cup\{0\}$.

The first two lemmas are technical properties from summation theory. 
Lemma 5.1. For any $C_{1} \geq 1$ and any $C_{2} \in R$ one has

$$
\sum_{k \in Z, k^{2} \geq C_{2}}\left(C_{1}+k^{2}\right)^{-1} \leq 5\left[C_{1}^{1 / 2}+\left(C_{2}\right)_{+}^{1 / 2}\right]^{-1}
$$

where $\left(C_{2}\right)_{+}=\max \left\{C_{2}, 0\right\}$.

Proof. Upon observing that $\left(C_{1}+k^{2}\right)^{-1} \leq 5\left(1+C_{1}^{1 / 2}+k\right)^{-2}$ for $C_{1} \geq 1$ and $k \geq 0$, one sees that the left-hand side of (5.3) is dominated by

$$
5 \sum_{k \in Z, k^{2} \geq C_{2}}\left(1+C_{1}^{1 / 2}+k\right)^{-2} \leq 5 \int_{C_{3}-1}^{\infty}\left(1+C_{1}^{1 / 2}+\theta\right)^{-2} d \theta
$$

where $C_{3}=\left(C_{2}\right)_{+}^{1 / 2}$. Evaluating the integral gives the required bound.

Lemma 5.2. The sum $\sum_{k \in Z^{2}}\left(1+|k|^{2}\right)^{-s}$ converges if and only if $s>1$.

Proof. In a standard fashion one compares this sum with the integral

$$
\int_{R^{2}}\left(1+x^{2}+y^{2}\right)^{-s} d x d y=\int_{0}^{2 \pi} \int_{0}^{\infty}\left(1+r^{2}\right)^{-s} r d r d \theta
$$

which is finite precisely when $s>1$.

Note that the first part of the next result is valid for a general bounded Lipschitz two-dimensional domain $\Omega$.

Lemma 5.3. Let the domain $\Omega$ be two-dimensional, and let $\left\{e_{m}\right\}_{m=1}^{\infty}$ denote a complete orthonormal set of eigenfunctions of $-\triangle$ with Neumann (or periodic in the case of a rectangle) boundary conditions. Then there exists a monotone function $\Theta(\lambda)>0$ satisfying $\Theta(\lambda) \rightarrow 0$ as $\lambda \rightarrow \infty$, such that for any $\lambda$ and $v \in H^{2}$ one has

$$
\sum_{\lambda_{m} \geq \lambda}\left|v_{m}\right| \leq \Theta(\lambda)\|v\|_{H^{2}}
$$

where $v_{m}=\left\langle v, e_{m}\right\rangle$ is the mth Fourier coefficient.

The same conclusion (5.4) holds for the three-dimensional cubic domain $\Omega_{3}=$ $(0,2 \pi)^{3}$ with either Neumann or periodic boundary conditions, and the particular orthonormal basis (5.1).

Proof. Consider first the case $\operatorname{dim} \Omega=2$ and fix $s$ to satisfy $\frac{1}{2}<s \leq \frac{3}{4}$. The linear growth $\lambda_{m} \sim C m$ implies that

$$
\sum_{m=1}^{\infty}\left(1+\lambda_{m}\right)^{-2 s}<\infty
$$

For all $v \in H^{2}$ one has $\left\|A^{s} v\right\| \leq K_{14}\|v\|_{H^{2}}$ for some $K_{14}$, and also

$$
\left\langle A^{s} v, e_{m}\right\rangle=\left\langle v, A^{s} e_{m}\right\rangle=\left(1+\lambda_{m}\right)^{s} v_{m}
$$


since $H^{2} \subset H^{2 s}=\mathscr{D}_{s}$, see Henry (1981). Therefore, by using first the Schwartz inequality and then the Parseval equality with $\lambda>0$ one obtains:

$$
\begin{aligned}
\sum_{\lambda_{m} \geq \lambda}\left|v_{m}\right| & \leq\left[\sum_{\lambda_{m} \geq \lambda}\left(1+\lambda_{m}\right)^{-2 s}\right]^{1 / 2}\left[\sum_{m=1}^{\infty}\left(1+\lambda_{m}\right)^{2 s}\left|v_{m}\right|^{2}\right]^{1 / 2} \\
& =\left[\sum_{\lambda_{m} \geq \lambda}\left(1+\lambda_{m}\right)^{-2 s}\right]^{1 / 2}\left\|A^{s} v\right\| \\
& \leq \Theta(\lambda)\|v\|_{H^{2}}
\end{aligned}
$$

where

$$
\Theta(\lambda)=\left[\sum_{\lambda_{m} \geq \lambda}\left(1+\lambda_{m}\right)^{-2 s}\right]^{1 / 2} K_{14} .
$$

In the case of the cube $\Omega_{3}=(0,2 \pi)^{3}$ with periodic boundary conditions the same proof works, but with $s$ chosen in the range $\frac{3}{4}<s \leq 1$. Here $\mathscr{D}_{s}=H^{2 s}$ for any $s \leq 1$, and one needs $s>\frac{3}{4}$ for the convergence of (5.5). (Recall the growth rate $\lambda_{m} \sim \mathrm{Cm}^{2 / 3}$.)

In the case of a cube with Neumann boundary conditions the proof must be modified: One has $\mathscr{D}_{s}=H^{2 s}$ only for $s \leq \frac{3}{4}$, however, the series (5.5) diverges for such $s$. But consider $s=1$. In place of (5.6) one obtains

$$
\begin{aligned}
\left(1+\lambda_{m}\right) v_{m} & =\left\langle v, A e_{m}\right\rangle=\left\langle v, e_{m}\right\rangle-\int_{\Omega_{3}} v \Delta e_{m} d x \\
& =\left\langle v, e_{m}\right\rangle-\int_{\Omega_{3}}(\Delta v) e_{m} d x+\int_{\partial \Omega_{3}} \frac{\partial v}{\partial n} e_{m} d \tilde{x} \\
& =\left\langle A_{*} v, e_{m}\right\rangle+\int_{\partial \Omega_{3}} \frac{\partial v}{\partial n} e_{m} d \tilde{x},
\end{aligned}
$$

where $A_{*}$ denotes the operator $I-\triangle$ extended to the domain $H^{2}$, and $d \tilde{x}$ denotes the surface element on $\partial \Omega_{3}$. As before one obtains a bound

$$
\sum_{\lambda_{m} \geq \lambda}\left(1+\lambda_{m}\right)^{-1}\left|\left\langle A_{*} v, e_{m}\right\rangle\right| \leq \Theta_{1}(\lambda)\|v\|_{H^{2}}
$$

involving the first term in (5.7), so it is sufficient to show

$$
\sum_{\lambda_{m} \geq \lambda}\left(1+\lambda_{m}\right)^{-1}\left|\int_{\partial \Omega_{3}} \frac{\partial v}{\partial n} e_{m} d \tilde{x}\right| \leq \Theta_{2}(\lambda)\|v\|_{H^{2}}
$$

with $\Theta(\lambda)=\Theta_{1}(\lambda)+\Theta_{2}(\lambda) \rightarrow 0$ as $\lambda \rightarrow \infty$.

We estimate the sum (5.8) where the integral is taken over only one face $S$ of the cube $\Omega_{3}$, say over the face

$$
S=(0,2 \pi)^{2} \times\{0\}
$$


in $x_{3}=0$. Writing a typical eigenfunction and eigenvalue as

$$
e_{k}(x)=\pi^{-3 / 2} \cos k x, \quad \lambda=|k|^{2}=k_{1}^{2}+k_{2}^{2}+k_{3}^{2}, \quad k \in Z^{3},
$$

one may use Lemma 5.1 to estimate $(5.8)$ by summing over $k_{3}$. With

$$
w\left(x_{1}, x_{2}\right)=-\left.\frac{\partial v\left(x_{1}, x_{2}, x_{3}\right)}{\partial x_{3}}\right|_{x_{3}=0}
$$

and

$$
\ell=\left(k_{1}, k_{2}\right) \in Z^{2}, \quad|\ell|^{2}=k_{1}^{2}+k_{2}^{2}, \quad y=\left(x_{1}, x_{2}\right) \in S
$$

one has

$$
\begin{aligned}
\pi^{-3 / 2} & \sum_{|k|^{2} \geq \lambda}\left(1+|\ell|^{2}+k_{3}^{2}\right)^{-1}\left|\int_{S} w(y) \cos \ell y d y\right| \\
& \leq 5 \pi^{-3 / 2} \sum_{|\ell|^{2} \geq \lambda}\left[\left(1+|\ell|^{2}\right)^{1 / 2}+\left(\lambda-|\ell|^{2}\right)_{+}^{1 / 2}\right]^{-1}\left|\int_{S} w(y) \cos \ell y d y\right| .
\end{aligned}
$$

Note $w$ belongs to $H^{1 / 2}(S)$ and satisfies

$$
\|w\|_{H^{1 / 2}(S)} \leq K_{15}\|v\|_{H^{2}}
$$

by the Trace Theorem.

To estimate the integral in (5.9) we employ the operator $\widetilde{A}=I-\widetilde{\triangle}$ where $\widetilde{\triangle}$ denotes the Laplacian with Neumann conditions on the two-dimensional face $S$. Fix a positive quantity $r \leq \frac{1}{4}$ and note that the functions $\tilde{e}_{\ell}(y)=\pi^{-1} \cos \ell y$ with $\ell \in Z^{2}$ form a complete orthonormal set of eigenvectors for this operator with eigenvalues $\left(1+|\ell|^{2}\right)$. Furthermore $w$ belongs to the domain of $\widetilde{A}^{r}$ and hence

$$
\int_{S} w(y) \cos \ell y d y=\pi\left\langle w, \tilde{e}_{\ell}\right\rangle=\pi\left(1+|\ell|^{2}\right)^{-r}\left\langle w, \widetilde{A}^{r} e_{\ell}\right\rangle=\pi\left(1+|\ell|^{2}\right)^{-r}\left\langle\tilde{v}, \tilde{e}_{\ell}\right\rangle
$$

where $\tilde{v}=\widetilde{A}^{r} w$. Inserting the formula (5.10) into (5.9), and using Parseval's equality again, one obtains the following upper bound for the right-hand side of (5.9):

$$
\begin{gathered}
\left\{25 \pi^{-1} \sum_{|\ell|^{2} \geq \lambda}\left[\left(1+|\ell|^{2}\right)^{1 / 2}+\left(\lambda-|\ell|^{2}\right)_{+}^{1 / 2}\right]^{-2}\left[1+|\ell|^{2}\right]^{-2 r}\right\}^{1 / 2}\|\tilde{v}\|_{L^{2}(S)} \\
=\widetilde{\Theta}(\lambda)\|\tilde{v}\|_{L^{2}(S)},
\end{gathered}
$$

where the above equality defines the quantity $\widetilde{\Theta}(\lambda)$. Next note that Lemma 5.2 implies that $\widetilde{\Theta}(\lambda)$ is finite for any $\lambda$ since $r>0$. We also note the terms in the convergent series defining $\widetilde{\Theta}(\lambda)$ each tend monotonically to zero as $\lambda \rightarrow \infty$, hence $\widetilde{\Theta}(\lambda) \rightarrow 0$ as $\lambda \rightarrow \infty$. We complete the estimate by observing that

$$
\|\tilde{v}\|_{L^{2}(S)}=\left\|\widetilde{A}^{r} w\right\|_{L^{2}(S)} \leq\left\|\widetilde{A}^{1 / 4} w\right\|_{L^{2}(S)}=\|w\|_{H^{1 / 2}(S)} \leq K_{15}\|v\|_{H^{2}},
$$

hence $(5.8)$ holds with $\Theta_{2}(\lambda)=\widetilde{\Theta}(\lambda) K_{15}$. 
The following result on the geometry of lattice points in two and three dimensions is central to the proof of PSA for $\Omega_{2}$ and $\Omega_{3}$.

Proposition 5.1. Fix $a=\left(a_{1}, a_{2}\right) \in R^{2}$ with each $a_{j}>0$, and consider points $a k=\left(a_{1} k_{1}, a_{2} k_{2}\right)$ where $k=\left(k_{1}, k_{2}\right) \in Z^{2}$. Then there exists $\xi>0$ such that for any $\kappa>1$ and $d>0$, there exists an arbitrarily large $\lambda$ satisfying the following two statements:

(1) Whenever $|a k|^{2},|a \ell|^{2} \in(\lambda-\kappa, \lambda+\kappa]$ with $\kappa, \ell \in Z^{2}$ one has either $k=\ell$ or $|a k-a \ell| \geq d ;$ and

(2) $|a k|^{2} \notin\left(\lambda-\frac{1}{2} \xi, \lambda+\frac{1}{2} \xi\right)$ for each $k \in Z^{2}$.

With $a=(1,1,1) \in R^{3}$, the corresponding result holds for lattice points $k \in Z^{3}$ in three-dimensional space with $\xi=1$.

Proposition 5.1 is a direct consequence of a Gap Theorem for families of quadratic forms and will be proved in the Appendix. Before proceeding to use it to verify PSA for $\Omega_{2}$ and $\Omega_{3}$, we contrast the two- and three-dimensional cases considered so far with the situation in four dimensions. Proposition 5.1 states that one can find arbitrarily large $\lambda$ such that the points of the lattice $a Z^{2}=\left\{\left(a_{1} k_{1}, a_{2} k_{2}\right): k_{1}, k_{2} \in Z\right\}$ in $R^{2}$, or $Z^{3}$ in $R^{3}$, which lie between the spheres of radii $(\lambda \pm \kappa)^{1 / 2}$ are "sparsely distributed." That is, these lattice points are separated by a minimum distance $d$ which can be chosen as large as desired. This property fails dramatically for the four-dimensional lattice $Z^{4}$, as the following result shows.

Proposition 5.2. For any quantities $\lambda \geq \kappa \geq 2$, there exist $k, \ell \in Z^{4}$ satisfying

$$
|k|^{2},|\ell|^{2} \in(\lambda-\kappa, \lambda+\kappa] \quad \text { and }|k-\ell|=1 .
$$

Proof. We use the fact that a nonnegative integer is the sum of three perfect squares if and only if it is not of the form $4^{a}(8 b+7)$, see Hardy and Wright (1979). Since $4^{a}(8 b+7)$ is always congruent to 0,4 , or 7 modulo 8 , it follows immediately that given any three consecutive nonnegative integers, at least one of them is a sum of three perfect squares. Therefore, given $\lambda$ and $\kappa$ as in the statement of the proposition there exists $\tilde{k} \in Z^{3}$ such that $|\tilde{k}|^{2} \in(\lambda-\kappa, \lambda+$ $\kappa-1]$, as the length of this interval is at least three. Writing $\tilde{k}=\left(\tilde{k}_{1}, \tilde{k}_{2}, \tilde{k}_{3}\right)$, define $k, \ell \in Z^{4}$ by

$$
k=\left(\tilde{k}_{1}, \tilde{k}_{2}, \tilde{k}_{3}, 0\right), \quad \ell=\left(\tilde{k}_{1}, \tilde{k}_{2}, \tilde{k}_{3}, 1\right) .
$$

Clearly, $k$ and $\ell$ have the required properties.

Remark. We do not make any direct use of Proposition 5.2 here, except to note that it implies our present techniques do not extend to the case of the four-dimensional domain $\Omega_{4}=(0,2 \pi)^{4}$. Indeed, with $\lambda, \kappa, k$, and $\ell=$ $k+(0,0,0,1)$ given as in Proposition 5.2, let $\rho(x)=\cos k x+\cos \ell x$ and 


$$
\begin{aligned}
& v(x)=\cos x_{4} \text { for } x \in \Omega_{4} \text {. Then } \rho=\left(P_{\lambda+\kappa}-P_{\lambda-\kappa}\right) \rho, \bar{v}=0, \text { and } \\
& \qquad \begin{aligned}
\left\|\left(P_{\lambda+\kappa}-P_{\lambda-\kappa}\right)\left(B_{v}-\bar{v} I\right)\left(P_{\lambda+\kappa}-P_{\lambda-\kappa}\right)\right\|_{\mathrm{op}} & \geq\|\rho\|^{-2}\left\langle\rho, B_{v} \rho\right\rangle \\
& =(2 \pi)^{-4} \int_{\Omega_{4}} \rho(x)^{2} v(x) d x=\frac{1}{2}
\end{aligned}
\end{aligned}
$$

as a simple calculation reveals. Thus PSA is false for $\Omega_{4}$, at least for Neumann and periodic boundary conditions.

There is a good reason for the failure of PSA for $\Omega_{4}$. In a companion paper to the present one, cf. Mallet-Paret and Sell (1988), we show that inertial manifolds themselves need not exist for reaction diffusion equations in $\Omega_{4}$. That is, there exists a smooth nonlinearity $f: \Omega_{4} \times R \rightarrow R$ such that the equation (3.6), with $\nu=1$, and with Neumann boundary conditions, possesses a finite dimensional global attractor $\mathfrak{A}$, yet does not possess an inertial manifold of any dimension.

We return to the domains $\Omega_{2}$ and $\Omega_{3}$, and use Proposition 5.1 to verify PSA.

Proposition 5.3. PSA holds for the domains

$$
\begin{aligned}
& \Omega_{2}=\left(0,2 \pi / a_{1}\right) \times\left(0,2 \pi / a_{2}\right), \\
& \Omega_{3}=(0,2 \pi)^{3},
\end{aligned}
$$

with either Dirichlet, Neumann, or periodic boundary conditions.

Proof. We consider only the rectangular domain $\Omega_{2}$ as the proof for $\Omega_{3}$ is similar. Consider first the case of periodic boundary conditions (the proof for Neumann conditions is essentially the same, but with Dirichlet conditions a slight modification, described below, is needed).

With $a=\left(a_{1}, a_{2}\right)$ fixed, let $\varepsilon>0$ and $\kappa>0$ be given and recall the definition of PSA given in $\S 4$. Fix $d>0$ sufficiently large so that

$$
\Theta\left(d^{2}\right)<\varepsilon
$$

where the function $\Theta$ is as in Lemma 5.3. With $\kappa$ and $d$ now given, let $\xi$ and $\lambda$ be as in Proposition 5.1; note that $\lambda$ can be selected to be arbitrarily large with the other quantities kept fixed. We observe immediately that (2) of Proposition 5.1 and the fact that the eigenvalues $\left\{\lambda_{m}\right\}_{m=1}^{\infty}$ of $-\triangle$ are the quantities $|a k|^{2}$, for $k \in Z^{2}$, yield the gap condition (4.3) in the definition of PSA. What remains to be proved, then, is the inequality (4.2).

Fix $v \in H^{2}$ and expand this function as a Fourier series in the eigenvalues of $-\triangle$ :

$$
v(x)=\sum_{k \in Z^{2}} v_{(k)} e^{i a k x} .
$$

(Observe the terms in this series are indexed here by $k \in Z^{2}$ rather than $m \geq 1$ as before; we write $v_{(k)}$ rather than $v_{m}$ to denote this.) Note the mean value

$$
\bar{v}=v_{(0)}
$$


of the function $v$, as well as the summability

$$
\sum\left|v_{(k)}\right|<\infty
$$

of the coefficients. Now fix a function

$$
\rho \in \operatorname{range}\left(P_{\lambda+\kappa}-P_{\lambda-\kappa}\right) \subset L^{2} ; \quad\|\rho\|=1 .
$$

Its Fourier expansion

$$
\rho(x)=\sum \rho_{(k)} e^{i a k x}
$$

involves only terms for which

$$
\lambda-\kappa<|a k|^{2} \leq \lambda+\kappa .
$$

One also has

$$
\sum\left|\rho_{(k)}\right|^{2}=\left(\operatorname{vol} \Omega_{2}\right)^{-1} \int_{\Omega_{2}}|\rho(x)|^{2} d x=\left(\operatorname{vol} \Omega_{2}\right)^{-1} .
$$

In view of the selfadjointness of the operator $B_{v}$, it is sufficient to show

$$
\left|\left\langle\rho,\left(B_{v}-\bar{v} I\right) \rho\right\rangle\right| \leq \varepsilon\|v\|_{H^{2}}
$$

to establish the required inequality (4.2).

The summability conditions (5.11) and (5.12) permit one to write term by term

$$
\begin{aligned}
\left\langle\rho,\left(B_{v}-\bar{v} I\right) \rho\right\rangle & =\sum_{j \neq 0} \sum_{k} \sum_{\ell} v_{(j)} \bar{\rho}_{(k)} \rho_{(\ell)} \int_{\Omega_{2}} e^{i a(j-k+\ell) x} d x \\
& =\left(\operatorname{vol} \Omega_{2}\right) \sum_{\substack{k, \ell \\
k \neq \ell}} v_{(k-\ell)} \bar{\rho}_{(k)} \rho_{(\ell)}
\end{aligned}
$$

where $k$ and $\ell$ are restricted to the ranges

$$
|a k|^{2},|a \ell|^{2} \in(\lambda-\kappa, \lambda+\kappa] \text {. }
$$

In addition, one has $k \neq \ell$ in the final summation in (5.13) as a result of the condition $j \neq 0$, the mean value of $v$ having been subtracted off. It follows from (1) of Proposition 5.1 that such $k$ and $\ell$ necessarily satisfy

$$
|a k-a \ell| \geq d .
$$

By a Young inequality, cf. Hewitt and Stromberg (1965, p. 397), one has the bound

$$
\begin{aligned}
\left(\operatorname{vol} \Omega_{2}\right) \sum_{\substack{k, \ell \\
k \neq \ell}} v_{(k-\ell)} \bar{\rho}_{(k)} \rho_{(\ell)} & \leq\left(\operatorname{vol} \Omega_{2}\right) \sum_{|a j| \geq d}\left|v_{(j)}\right| \sum\left|\rho_{(k)}\right|^{2} \\
& =\sum_{|a j| \geq d}\left|v_{(j)}\right| \leq \Theta\left(d^{2}\right)\|v\|_{H^{2}} \leq \varepsilon\|v\|_{H^{2}}
\end{aligned}
$$

as required. This completes the proof in the case of periodic boundary conditions. The proof in the case of Neumann conditions is virtually the same as that above; we omit it. 
When Dirichlet conditions are taken, the following modification is made. The function $v$ is expanded as a Fourier series

$$
v(x)=\sum v_{(k)} \cos a k x
$$

in terms of the eigenfunctions of $-\triangle$ with Neumann conditions. The function $\rho$ is expanded using the Dirichlet eigenfunctions

$$
\rho(x)=\sum \rho_{(k)} \sin a k x .
$$

Lemma 5.3 (which does not generally hold for the Dirichlet expansion) gives the appropriate summability condition (5.4) for the coefficients $v_{(k)}$. One also sees that a typical term

$$
v_{(j)} \bar{\rho}_{(k)} \rho_{(\ell)} \int_{\Omega_{2}} \cos a j x \sin a k x \sin a \ell x d x
$$

in the summation (5.12) vanishes unless $j= \pm k \pm \ell$. From here on the proof proceeds much as before.

\section{APPENDIX A: GAP THEOREMS FOR FAMILIES OF QUADRATIC FORMS}

Our goal in this Appendix is to give a proof of Proposition 5.1. This proposition, which is a statement on the geometry of certain lattice points in $R^{2}$ and $R^{3}$, is closely connected to properties of quadratic forms with rational coefficients. The property we need to derive is the following Gap Theorem.

Special Gap Theorem. Let $\mathscr{T}$ be a finite collection of functions $T$ of the form

$$
T\left(k_{1}, k_{2}\right)=a k_{1}^{2}+b k_{1} k_{2}+c k_{2}^{2}+s k_{1}+t k_{2}+u,
$$

with rational coefficients and negative discriminant, i.e.,

$$
a, b, c, s, t, u \in Q, \quad b^{2}-4 a c<0 .
$$

Then given any $h>0$ there exist arbitrarily large $m$ such that

$$
T\left(k_{1}, k_{2}\right) \notin[m, m+h] \text { for each } T \in \mathscr{T} \text { and } k_{1}, k_{2} \in Z \text {. }
$$

We will, in fact, prove a stronger result, the General Gap Theorem, below. This stronger version allows the discriminant of the quadratic form to be either negative or to assume selected positive values.

These gap theorems are extensions of a result of Richards (1982) who proved a similar result for a single value of the discriminant. Actually Richards showed that the gaps $h$ grow with $m$ at a rate $\geq$ constant $\log m$. With a little extra work, we could obtain the same bound in the more general theorem above. We have not spelled out this stronger result, since we do not need it for our purposes.

We begin with a discussion of the number-theoretic function $\left(\frac{m}{n}\right)$, called either Legendre's symbol, Jacobi's symbol, or Kronecker's symbol, depending on the context in which it is used, see Hardy and Wright (1979, Chapter 6) and Watson (1960, Chapter 3). Let $p>0$ be a prime number and let $m \in Z$ satisfy 
$p \nmid m$, that is, $p$ does not divide $m$. The integer $m$ is called a quadratic residue of $p$ in case $m \equiv k^{2}(p)$ for some integer $k$. (The notation $m_{1} \equiv m_{2}(n)$ denotes equivalence of $m_{1}$ and $m_{2}$ modulo $n$, that is, $n \mid\left(m_{1}-m_{2}\right)$.) If $p \nmid m$ and the equation $m \equiv k^{2}(p)$ has no solution $k$, then $m$ is called a quadratic nonresidue. Set

$$
\left(\frac{m}{p}\right)= \begin{cases}1 & \text { if } p+m \text { and } m \text { is a quadratic residue } \\ -1 & \text { if } p+m \text { and } m \text { is a quadratic nonresidue }\end{cases}
$$

where $p$ is odd. This is Legendre's symbol. For the even prime $p=2$, and $m$ odd, set

$$
\left(\frac{m}{2}\right)=(-1)^{\left(m^{2}-1\right) / 8} \text {. }
$$

Jacobi's symbol $\left(\frac{m}{n}\right)$ is now defined for all $m, n \in Z$ with $m \neq 0, n \neq 0$, and greatest common divisor $\operatorname{gcd}(m, n)=1$, by extending the above formulas uniquely so that

$$
\left(\frac{m}{n_{1} n_{2}}\right)=\left(\frac{m}{n_{1}}\right)\left(\frac{m}{n_{2}}\right)
$$

and $\left(\frac{m}{ \pm 1}\right)=1$. Clearly $\left(\frac{m}{n}\right)=\left(\frac{m}{|n|}\right)= \pm 1$ for all such $m$ and $n$. It can be proved that Jacobi's symbol is multiplicative in the numerator, that is

$$
\left(\frac{m_{1} m_{2}}{n}\right)=\left(\frac{m_{1}}{n}\right)\left(\frac{m_{2}}{n}\right) \text {, }
$$

and also that

$$
\left(\frac{m_{1}}{n}\right)=\left(\frac{m_{2}}{n}\right) \quad \text { if } m_{1} \equiv m_{2}(n) \text { and } n \not \equiv 2(4) .
$$

(Note that $\left(\frac{m+2}{2}\right) \neq\left(\frac{m}{2}\right)$ in general, but $\left(\frac{m+8}{2}\right)=\left(\frac{m}{2}\right)$.) Further, the celebrated Quadratic Reciprocity Law of Gauss states that

$$
\text { (A.4) } \quad\left(\frac{m}{n}\right)=(-1)^{((m-1) / 2)((n-1) / 2)}\left(\frac{n}{m}\right), \quad m, n \text { odd and positive. }
$$

Also one has

$$
\begin{array}{rlrl}
\left(\frac{-1}{n}\right) & =(-1)^{(n-1) / 2}, & & n \text { odd }, \\
\left(\frac{2}{n}\right) & =(-1)^{\left(n^{2}-1\right) / 8}, \quad n \text { odd. }
\end{array}
$$

In all the above formulas when we write Jacobi's symbol it is assumed the arguments lie in the domain of definition: $m \neq 0, n \neq 0$, and $\operatorname{gcd}(m, n)=1$.

In the theory of quadratic forms the numerator $m$ is typically a discriminant $m=b^{2}-4 a c$, and as such satisfies $m \equiv 0$ or $1(4)$. In this context $\left(\frac{m}{n}\right)$ is called Kronecker's symbol, and one has

$$
\left(\frac{m}{n_{1}}\right)=\left(\frac{m}{n_{2}}\right) \quad \text { if } n_{1} \equiv n_{2}(m) \text { and } m \equiv 0 \text { or } 1(4)
$$

provided, as above, $m \neq 0, n_{j} \neq 0$, and $\operatorname{gcd}\left(m, n_{j}\right)=1, j=1,2$. The formula (A.6) follows easily from the properties of Jacobi's symbol, in particular from (A.3) and the Quadratic Reciprocity Law (A.4). 
Lemma A.1. Let $D \subset Z$ be a finite nonempty set of integers with the property that $\Pi_{d \in A} d$ is not a perfect square whenever $A \subset D$ has odd cardinality. Then there exists an integer $r \neq 0$ such that

$$
\operatorname{gcd}(d, r)=1 \quad \text { and } \quad\left(\frac{d}{r}\right)=-1
$$

for each $d \in D$.

Note that if $D$ is a nonempty finite set of negative integers, then the hypotheses of the above lemma are satisfied since the quantity $\Pi_{d \in A} d$ is negative, hence not a perfect square.

Proof. Denote $D=\left\{d_{i}\right\}_{i=1}^{m}$ and let $\left\{p_{j}\right\}_{j=0}^{n}$ be all the primes which occur as factors in the various $d_{i}$, together with $p_{0}=-1$. Include $p_{1}=2$ even if this prime does not occur as a factor. One may write

$$
d_{i}=\prod_{j=0}^{n} p_{j}^{\alpha_{i j}}
$$

for some nonnegative integers $\alpha_{i j}$. (Note that $\alpha_{i 0}$ is only determined modulo 2.) The hypothesis on products $\Pi_{d \in A} d$ of odd order says precisely that whenever $\left\{\beta_{i}\right\}_{i=1}^{m}$ are integers whose sum $\sum_{i=1}^{m} \beta_{i}$ is odd, then the sum $\sum_{i=1}^{m} \beta_{i} \alpha_{i j}$ is odd for some $j$. Equivalently, in terms of vector products one has

$$
\beta^{*} \varepsilon \not \equiv 0 \Longrightarrow \beta^{*} \alpha \not \equiv 0
$$

where $\alpha=\left(\alpha_{i j}\right), \beta=\left(\beta_{i}\right)$, and $\varepsilon=\operatorname{col}(1, \ldots, 1) \in Z_{2}^{m}$ are the matrix and column vectors with entries in the finite field $Z_{2}, *$ denotes the transpose, and $\equiv$ denotes equivalence in $Z_{2}$.

From the implication (A.7), and the fact that $Z_{2}$ is a field, it follows that there exists a column vector $\gamma \in Z_{2}^{n+1}$ such that $\alpha \gamma \equiv \varepsilon$, that is, $\sum_{j=0}^{n} \alpha_{i j} \gamma_{j}$ is an odd integer for each $i$. It is sufficient now that $r$ should satisfy

$$
\operatorname{gcd}\left(p_{j}, r\right)=1 \quad \text { and } \quad\left(\frac{p_{j}}{r}\right)=(-1)^{\gamma_{j}}
$$

for each $j$, for then one has $\operatorname{gcd}\left(d_{i}, r\right)=1$ (for each $i$ ) and

$$
\left(\frac{d_{i}}{r}\right)=\prod_{j=0}^{n}\left(\frac{p_{j}}{r}\right)^{\alpha_{i j}}=\prod_{j=0}^{n}(-1)^{\alpha_{i j} \gamma_{j}}=-1
$$

as required.

For $p_{0}=-1$ and $p_{1}=2$ it follows from (A.5) that the equations (A.8) are satisfied by an appropriate choice of one of the four conjugacy classes $r \equiv$ $1,3,5$, or 7(8) for $r$. With the value of $r$ (modulo 8) now fixed, the equations (A.8) for the remaining $p_{j}$ now reduce, by the Quadratic Reciprocity Law (A.4), to achieving a prescribed value \pm 1 for each $\left(\frac{r}{p_{j}}\right)$. This in turn reduces to prescribing values $r \equiv r_{j}\left(p_{j}\right)$ along with the value $r \equiv 1,3,5$, or 7(8) already 
assigned. The existence of $r$ satisfying these congruences follows directly from the Chinese Remainder Theorem.

We now study quadratic forms

$$
T\left(k_{1}, k_{2}\right)=a k_{1}^{2}+b k_{1} k_{2}+c k_{2}^{2}
$$

with integer coefficients $a, b, c \in Z$. The discriminant of $T$ is the familiar quantity

$$
d=b^{2}-4 a c
$$

which necessarily satisfies $d \equiv 0$ or $1(4)$. It is useful here to introduce notation which indicates the number of times a prime number appears as a factor in an integer. If $p>0$ is prime we write

$$
\left.p\right|_{\mathrm{e}} n
$$

to mean either $n=p^{\alpha} m$ where $\alpha$ is even and $p \nmid m$, or else $n=0$. (Note that $\alpha=0$ is permitted, so $\left.p\right|_{e} n$ holds if $p \nmid n$.) We write

$$
\left.p\right|_{0} n
$$

to mean $n=p^{\alpha} m$ where $\alpha$ is odd and $p \nmid m$. (The e and o above means that $p$ divides $n$ an even or odd number of times, respectively.)

Lemma A.2. Let $T$ be a quadratic form (A.9) with integer coefficients and discriminant $d=b^{2}-4 a c$, and let $p$ be an odd prime satisfying $p \nmid d$ and $\left(\frac{d}{p}\right)=-1$. Then

$$
\left.p\right|_{\mathrm{e}} T\left(k_{1}, k_{2}\right)
$$

for any $k_{1}, k_{2} \in Z$.

Proof. It is enough to show that $p \mid k_{1}$ and $p \mid k_{2}$ whenever $p \mid T\left(k_{1}, k_{2}\right)$, as this yields a factor $p^{2}$ of $T\left(k_{1}, k_{2}\right)$. Repeated factorings of $p^{2}$ give the result. We argue by contradiction. Assume then $p \mid T\left(k_{1}, k_{2}\right)$ but $p \nmid k_{2}$. Observe that $p \nmid a$, for otherwise $d \equiv b^{2}(p)$ would be either a multiple or a quadratic residue or $p$, contradicting $\left(\frac{d}{p}\right)=-1$. Working in the field $Z_{p}$ one has

$$
a k_{1}^{2}+b k_{1} k_{2}+c k_{2}^{2} \equiv 0(p) \text {. }
$$

Multiplying by $4 a\left(k_{2}^{2}\right)^{-1} \not \equiv 0(p)$, writing $k_{0} \equiv k_{1} k_{2}^{-1}(p)$, and completing the square yields

$$
\left(2 a k_{0}+b\right)^{2}+4 a c-b^{2} \equiv 0(p) .
$$

This implies $d$ is either a multiple or a quadratic residue of $p$, again contradicting $\left(\frac{d}{p}\right)=-1$.

The following result, a direct generalization of work of Richards (1982), concerns simultaneous gaps in the ranges of families of quadratic forms. 
General Gap Theorem. Let $D \subset Z$ be a set of integers satisfying the hypotheses of Lemma A.1 and fix a quantity $h>0$. Then there exist arbitrarily large $m$ with the following property: If $T$ is any quadratic form (A.9) with integer coefficients, and with discriminant $d=b^{2}-4 a c \in D$, then

$$
T\left(k_{1}, k_{2}\right) \notin[m, m+h] \quad \text { for each } k_{1}, k_{2} \in Z .
$$

Proof. Assume without loss of generality that $d \equiv 0$ or 1(4) for each $d \in D$ (since any number of the form $b^{2}-4 a c$ satisfies such a congruence) and define $\delta$ to be the least common multiple $\delta=\operatorname{lcm}\{|d|: d \in D\}$. Let $r$ be as in Lemma A.1, and note that

$$
\operatorname{gcd}(\delta, r)=1
$$

since $\operatorname{gcd}(d, r)=1$ for each $d \in D$. Fix $A>0$ large enough that

$$
|r+\delta j| \leq A \quad \text { whenever } 0 \leq j \leq n
$$

and let $P$ be the product

$$
P=\prod p^{1+\alpha}
$$

where the product is taken over all primes $p$ with $p \nmid \delta$ and $p^{\alpha} \leq A<p^{1+\alpha}$ for some $\alpha>0$. Let $m$ be any integer satisfying the congruence

$$
\delta m \equiv r(P) .
$$

We claim that $m$ satisfies the conclusion of the General Gap Theorem. In view of Lemma A.2, it is enough to show that whenever $0 \leq j \leq n$ and $d \in D$, there exists a prime number $p$ satisfying

$$
\begin{aligned}
& p \nmid d \text { and }\left(\frac{d}{p}\right)=-1, \\
& \left.p\right|_{\mathrm{o}}(m+j) .
\end{aligned}
$$

Now $\left(\frac{d}{r+\delta j}\right)=\left(\frac{d}{r}\right)=-1$ by (A.6), so factoring $r+\delta j$ and using the multiplicative property (A.2) for the denominator of Kronecker's symbol shows there exists a prime $p$ satisfying (A.12) with

$$
\left.p\right|_{\mathrm{o}}(r+\delta j) \text {. }
$$

By (A.11) one has $p \leq A$; one also has $p \nmid \delta$, otherwise $p$ would be a common factor of $r+\delta j$ and $\delta$, hence $p \mid r$ which contradicts (A.10). Thus $p$ occurs as a factor in $P$, and one sees from the definition of $P$ that it occurs with a greater multiplicity as a factor of $P$ than as a factor of $r+\delta j$. With this observation and the congruence

$$
\delta(m+j) \equiv r+\delta j(P),
$$

it follows that $p$ divides $\delta(m+j)$ and $r+\delta j$ the same number of times. Thus $\left.p\right|_{\mathrm{o}} \delta(m+j)$, and hence $\left.p\right|_{\mathrm{o}}(m+j)$ as claimed.

The Special Gap Theorem is now a corollary of the last result. 
Proof of the Special Gap Theorem. If for each $T \in \mathscr{T}$ the coefficients $a, b$, and $c$ are integers and $s=t=u=0$, then the result follows directly from the General Gap Theorem. More generally, we shall reduce the problem to considering this case.

With $T \in \mathscr{T}$ as in the statement of the Special Gap Theorem, there exist rational numbers $e_{1}, e_{2}$, and $e_{3}$ (depending on $T$ ) such that

$$
T\left(x_{1}+e_{1}, x_{2}+e_{2}\right)-e_{3}=a x_{1}^{2}+b x_{1} x_{2}+c x_{2}^{2},
$$

for all rational numbers $x_{1}, x_{2}$. To be specific let $n$ and $\ell$ be positive integers such that

$$
n e_{1}, n e_{2}, \ell a, \ell b, \ell c \in Z \quad \text { for each } T \in \mathscr{T}
$$

and consider arguments of the form $x_{j}=i_{j} / n$ for integers $i_{1}$ and $i_{2}$. (Observe that all integers $k_{j}=i_{j} / n+e_{j}$ can be so obtained.) Now

$$
\left[T\left(\frac{i_{1}}{n}+e_{1}, \frac{i_{2}}{n}+e_{2}\right)-e_{3}\right] \ell n^{2}=\ell a i_{1}^{2}+\ell b i_{1} i_{2}+\ell c i_{2}^{2}
$$

has integer coefficients and a negative discriminant. Therefore, with

$$
h_{0}=\left(h+\max _{\mathscr{T}} e_{3}-\min _{\mathscr{T}} e_{3}\right) \ell n^{2}
$$

and by the General Gap Theorem, there exist arbitrarily large $m_{0}$ such that the right-hand side of (A.13) does not take values in $\left[m_{0}, m_{0}+h_{0}\right]$ for any $T \in \mathscr{T}$ and $i_{1}, i_{2} \in Z$. But this easily implies the result (A.1) with

$$
m=\frac{m_{0}}{\ell n^{2}}+\max _{\mathscr{T}} e_{3} \text {. }
$$

We are now prepared for the final step!

Proof of Proposition 5.1. Consider the two-dimensional case first. Let $a=$ $\left(a_{1}, a_{2}\right), \kappa>1$, and $d>0$ be fixed as in the statement of the proposition. We shall use the lattice $a Z^{2}$ where

$$
a Z^{2}=\left\{\left(a_{1} k_{1}, a_{2} k_{2}\right): k_{1}, k_{2} \in Z\right\} .
$$

We need to determine $\xi>0$ and show that there exists arbitrarily large $\lambda$ such that

Whenever $|a k|^{2},|a \ell|^{2} \in(\lambda-\kappa, \lambda+\kappa]$ with $k, \ell \in Z^{n}$ one has either $k=\ell$ or $|a k-a \ell| \geq d$,

and

$$
|a k|^{2} \notin\left(\lambda-\frac{1}{2} \xi, \lambda+\frac{1}{2} \xi\right) \quad \text { for each } k \in Z^{n}
$$

for $n=2$. For a sufficiently large quantity $\mu$, to be determined later, we consider the family of disjoint annuli

$$
N_{m}^{\mu}=\left\{x \in R^{2}: \mu+(2 m-1) \kappa<|x|^{2} \leq \mu+(2 m+1) \kappa\right\},
$$


where $0 \leq m \leq J=\operatorname{Int}\left(\mu^{1 / 2}\right)$, the integer part of $\mu^{1 / 2}$, and such that the union

$$
N^{\mu}=\bigcup_{m=0}^{J} N_{m}^{\mu}=\left\{x \in R^{2}: \mu-\kappa<|x|^{2} \leq \mu+(2 J+1) \kappa\right\}
$$

is also an annulus with inner radius $(\mu-\kappa)^{1 / 2}$. By using the relationship $(1+x)^{1 / 2}=1+\frac{1}{2} x+O\left(|x|^{2}\right)$ for small $x$ we see that the thickness of $N^{\mu}$ is

$$
\begin{aligned}
(\mu+(2 J+1) \kappa)^{1 / 2}-(\mu-\kappa)^{1 / 2} & =\mu^{1 / 2}\left[1+\mu^{-1}\left(J+\frac{1}{2}\right) \kappa-1+\frac{1}{2} \mu^{-1} \kappa+O\left(\mu^{-2}\right)\right] \\
& =\mu^{-1 / 2} J \kappa+\mu^{-1 / 2} \kappa+O\left(\mu^{-3 / 2}\right) \\
& =\kappa+0\left(\mu^{-1 / 2}\right) \quad \text { as } \mu \rightarrow \infty,
\end{aligned}
$$

and is asymptotically constant for large $\mu$. Hence the number of points of the lattice $a Z^{2}$ in the annulus $N^{\mu}$ is

$$
\operatorname{card}\left(N^{\mu} \cap a Z^{2}\right)=0\left(\text { meas } N^{\mu}\right)=0\left(\mu^{1 / 2}\right) \quad \text { as } \mu \rightarrow \infty .
$$

We wish to prove (A.14) and (A.15) for some $\lambda$ between $\mu$ and $\mu+2 J \kappa$. Let

$$
\widetilde{N}_{m}^{\mu}=N_{m-1}^{\mu} \cup N_{m}^{\mu} \cup N_{m+1}^{\mu}
$$

denote the union of three adjacent annuli, for $1 \leq m \leq J-1$ and suppose there exist points

$$
a k, a \ell \in \widetilde{N}_{m}^{\mu} \cap a Z^{2}, \quad 0<|a k-a \ell|<d
$$

for some $m$. Writing $j=\ell-k$, we have

$$
|a \ell|^{2}=|a k|^{2}+2(a k) \cdot(a j)+|a j|^{2}
$$

and so

$$
|(a k) \cdot(a j)| \leq\left.\frac{1}{2}|| a \ell\right|^{2}-\left.|a k|^{2}\left|+\frac{1}{2}\right| a j\right|^{2}<3 \kappa+\frac{1}{2} d^{2} .
$$

That is, the lattice point $a k$ belongs to the strip

$$
S_{j}=\left\{x \in R^{2}:|x \cdot(a j)|<3 \kappa+\frac{1}{2} d^{2}\right\},
$$

of fixed width $w_{j}$ independent of $\mu$, for some $j \in Z^{2}$ satisfying

$$
0<|a j|<d .
$$

Let $S$ denote the finite union of these strips, i.e.,

$$
S=\bigcup_{0<|a j|<d} S_{j} .
$$

Because the strips $S_{j}$ are symmetric about the origin and the annulus $N^{\mu}$ is centered at the origin, meas $\left(S_{j} \cap N^{\mu}\right)$ is approximately twice the product of the width of $S_{j}$ and the thickness of $N^{\mu}$, i.e.,

$$
\operatorname{meas}\left(S_{j} \cap N^{\mu}\right)=2 w_{j} \kappa+O\left(\mu^{-1 / 2}\right) \quad \text { as } \mu \rightarrow \infty .
$$


Since indices $j$ range over a finite set given by (A.17) it follows that the total number of possible lattice points $a k$ above, for all $m$, is of bounded order for large $\mu$ :

$$
\operatorname{card}\left(S \cap N^{\mu} \cap a Z^{2}\right)=0\left(\operatorname{meas}\left(S \cap N^{\mu}\right)\right)=0(1) \quad \text { as } \mu \rightarrow \infty .
$$

Thus for large $\mu$ and for most $m$ the set $S \cap \tilde{N}_{m}^{\mu} \cap a Z^{2}$ is empty and (A.14) holds for a range of $\lambda$. More precisely, let

$$
M^{\mu}=\left\{m \in Z: 1 \leq m \leq J-1 \text { and } S \cap \widetilde{N}_{m}^{\mu} \cap a Z^{2}=\varnothing\right\} .
$$

Then for each $m \in M^{\mu}$, (A.14) holds for any $\lambda$ satisfying

$$
\lambda \in(\mu+(2 m-1) \kappa, \mu+(2 m+1) \kappa] .
$$

Furthermore,

$$
\text { card } M^{\mu} \sim \mu^{1 / 2} \quad \text { as } \mu \rightarrow \infty
$$

since it follows from (A.18) that the number of $m$ between 1 and $J-1$ which are not in $M^{\mu}$ is bounded for large $\mu$.

Now to ensure (A.15) holds for some such $\lambda$, we note that (A.20)

$$
\left(\operatorname{card} M^{\mu}\right)^{-1} \sum_{m \in M^{\mu}} \operatorname{card}\left(N_{m}^{\mu} \cap a Z^{2}\right) \leq\left(\operatorname{card} M^{\mu}\right)^{-1} \operatorname{card}\left(N^{\mu} \cap a Z^{2}\right)=0(1)
$$

as $\mu \rightarrow \infty$, by (A.16) and (A.19). Let $C>0$ be an upper bound for the quantities in (A.20). Then for each large $\mu$ there exists at least one $m \in M^{\mu}$ for which

$$
\operatorname{card}\left(N_{m}^{\mu} \cap a Z^{2}\right) \leq C .
$$

That is, the interval $(\mu+(2 m-1) \kappa, \mu+(2 m+1) \kappa]$ contains at most $C$ points of the form $|a k|^{2}$, and so must contain a subinterval $I$ of length at least $2 \kappa(1+C)^{-1}$ with no such points in its interior. One sees therefore, that by choosing $\lambda$ as the midpoint of $I$, and $\xi=2(1+C)^{-1} \leq 2 \kappa(1+C)^{-1}$ (A.15) is fulfilled. As noted, (A.14) also holds, so the result in the two-dimensional case is proved.

The proof in the three-dimensional case, with $a=(1,1,1)$, bears some similarity to the above, but there are some significant differences. Because the quantities $|a k|^{2}=|k|^{2}$ are all integers, (A.15) holds immediately with $\xi=1$ whenever $\lambda$ is half of an odd integer, that is $\lambda-\frac{1}{2} \in Z$. We consider then only such $\lambda$. To verify (A.14), we need only examine a single annular region

$$
N_{0}^{\lambda}=\left\{x \in R^{3}: \lambda-\kappa<|x|^{2} \leq \lambda+\kappa\right\} .
$$

As in the two-dimensional case, if (A.15) fails for this $\lambda$, then

$$
S \cap N_{0}^{\lambda} \cap Z^{3} \neq \varnothing
$$


where $S$ now is the finite union of slabs defined by $|x \cdot j|<\kappa+d / 2$ and $j \in Z^{3}$ satisfies $0<|j|<d$. Now consider a point $k$ in the intersection (A.21). As $k \in S \cap Z^{3}$, one has

$$
k \cdot j=n
$$

for some such $j$ and some integer $n$ satisfying $|n|<\kappa+\frac{1}{2} d^{2}$. Since $j \neq 0$ we can assume, without loss of generality, that $j_{3} \neq 0$. One may then solve (A.22) for $k_{3}$ to obtain

$$
k_{3}=-j_{3}^{-1}\left(k_{1} j_{1}+k_{2} j_{2}-n\right),
$$

and hence

$$
|k|^{2}=k_{1}^{2}+k_{2}^{2}+j_{3}^{-2}\left(k_{1} j_{1}+k_{2} j_{2}-n\right)^{2} .
$$

Denoting the right-hand side of the formula (A.23) by $T_{j, n}\left(k_{1}, k_{2}\right)$, one has now

$$
T_{j, n}\left(k_{1}, k_{2}\right) \in(\lambda-\kappa, \lambda+\kappa]
$$

since $k \in N_{0}^{\lambda}$. Now as the indices $j$ and $n$ range over finite sets, and the functions $T_{j, n}$ are all positive definite, the hypotheses of the Special Gap Theorem are fulfilled. With $h=2+2 \kappa$ and $m$ as in the statement of the Special Gap Theorem, one sees that $(\lambda-\kappa, \lambda+\kappa] \subset[m, m+h]$ for some $\lambda$ such that $\lambda-\frac{1}{2} \in Z$, and so (A.24) is impossible. As $m$ can be chosen arbitrarily large, the proof is now complete.

\section{REFERENCES}

Adams, R. A. (1975), Sobolev spaces, Academic Press, New York.

Billotti, J. E. and J. P. LaSalle (1971), Dissipative periodic processes, Bull. Amer. Math. Soc. 77, 1082-1088.

Constantin P. and C. Foias (1985), Global Lyapunov exponents, Kaplan-Yorke formulas and the dimension of the attractor for $2 D$ Navier-Stokes equation, Comm. Pure Appl. Math. 38, 1-27.

Constantin, P., C. Foias, B. Nicolaenko, and R. Temam (1986), Nouveaux résultats sur les varietés inertielles pour les équations différentielles dissipatives, C. R. Acad. Sci. Paris I 302, 375-378. , (1988), Integral manifolds and inertial manifolds for dissipative partial differential equations, Inst. Appl. Math. Sci. Comp. Preprint, Indiana Univ. , (1989), Spectral barriers and inertial manifolds for dissipative partial differential equations, J. Dynamics and Differential Equations 1 (to appear).

Constantin, P., C. Foias, and R. Temam (1985), Attractors representing turbulent flows, Mem. Amer. Math. Soc., no. 314, Amer. Math. Soc., Providence, RI.

Edmunds D. E. and W. D. Evans (1987), Spectral Theory and Differential Operators, Oxford Mathematical Monographs, Oxford, U.K.

Fenichel, N. (1971), Persistence and smoothness of invariant manifolds for flows, Indiana Univ. Math. J. 21, 193-226.

Foias, C., O. Manley, and R. Temam (1986), Attractors for the Bernard problem: Existence and physical bounds of their fractal dimension (to appear). 
Foias, C., B. Nicolaenko, G. R. Sell, and R. Temam (1985), Varietes inertielles pour l'equation de Kuramoto-Sivashinsky, C. R. Acad. Sci. Paris Sér. I Math. 301, 285-288.

_., (1988), Inertial manifold for the Kuramoto Sivashinsky equation, J. Math. Pures Appl. (to appear).

Foias, C., G. R. Sell, and R. Temam (1985), Varietes inertielles des equations differentielles dissipatives, C. R. Acad. Sci. Paris Sér. I Math. 301, 139-141.

, (1986), Inertial manifolds for nonlinear evolutionary equations, IMA Preprint No. 234, March 1986. Also in J. Differential Equations 73 (1988), 309-353.

Foias, C., G. R. Sell, and E. Titi (1988), Exponential tracking and approximation of inertial manifolds for dissipative equations, Inst. Appl. Math. Sci. Comp. Preprint, Indiana Univ.

Foias, C. and R. Temam (1979), Some analytic and geometric properties of the solutions of the Navier-Stokes equations, J. Math. Pures Appl. 58, 339-368.

Hale, J. K. (1988), Asymptotic behavior of dissipative systems, Amer. Math. Soc., Providence, RI.

Hale, J. K., L. T. Magalhaes, and W. M. Oliva (1984), An introduction to infinite dimensional and dynamical systems-geometric theory, Appl. Math. Sciences, No. 47, Springer-Verlag, New York.

Hardy, G. H. and E. M. Wright (1979), An introduction to the theory of numbers, 5th ed., Oxford Press, Oxford.

Hartman, P. (1964), Ordinary differential equations, Wiley, New York.

Henry, D. (1981), Geometric theory of semilinear parabolic equations, Lecture Notes in Math., No. 840, Springer-Verlag, New York.

Hewitt, E. and K. Stromberg (1965), Real and abstract analysis, Graduate Texts in Math., No. 25, Springer-Verlag, New York.

Hirsch, M., C. Pugh, and M. Shub (1977), Invariant manifolds, Lecture Notes in Math., No. 583, Springer-Verlag, New York.

Kamaev, D. A. (1981), Hopf's conjecture for a class of chemical kinetics equations, J. Soviet Math. 25, 836-849.

Mallet-Paret, J. (1976), Negatively invariant sets of compact maps and an extension of a theorem of Cartwright, J. Differential Equations 22, 331-348.

Mallet-Paret, J. and G. R. Sell (1988), Counterexamples to the existence of inertial manifolds.

Mañé, R. (1977), Reduction of semilinear parabolic equations to finite dimensional $C^{1}$ flows, Geometry and Topology, Lecture Notes in Math., No. 597, Springer-Verlag, New York, 361-378. , (1981), On the dimension of the compact invariant sets of certain nonlinear maps, Lecture Notes in Math., No. 898, Springer-Verlag, New York, 230-242.

Mora, X. (1983), Finite dimensional attracting manifolds in reaction diffusion equations, Contemp. Math. 17, 353-360.

Mora, X. and J. Solà-Morales (1988), The singular limit dynamics of semilinear damped wave equations, Preprint, Univ. Autònoma Barcelona.

Nicolaenko, B., B. Scheurer, and R. Temam (1985), Some global dynamical properties of the Kuramoto Sivashinsky equations: nonlinear stability and attractors, Physica D 16D, 155-183. , (1987), Some global dynamical properties of a class of pattern formation equations, IMA Preprint No. 381.

Pazy, A. (1983), Semigroups of linear operators and applications to partial differential equations, Appl. Math. Sci., Vol. 44, Springer-Verlag, New York.

Pliss, V. A. (1964), A reduction principle in the theory of the stability of motion, Izv. Akad. Nauk SSSR Mat. Ser. 28, 1297-1324.

Richards, I. (1982), On the gaps between numbers which are the sum of two squares, Adv. Math. 46, 1-2.

Sacker, R. J. (1969), A perturbation theorem for invariant manifolds and Hölder continuity, J. Math. Mech. 18, 705-762.

Sacker, R. J. and G. R. Sell (1980), The spectrum of an invariant submanifold, J. Differential Equations 38, 135-160.

Temam, R. (1988), Infinite dimensional dynamical systems in mechanics and physics, SpringerVerlag, New York.

Watson, G. L. (1960), Integral quadratic forms, Cambridge Press, Cambridge, England. 
ABSTRACT. In this paper we show that the scalar reaction diffusion equation

$$
u_{t}=\nu \Delta u+f(x, u), \quad u \in R
$$

with $x \in \Omega_{n} \subset R^{n} \quad(n=2,3)$ and with Dirichlet, Neumann, or periodic boundary conditions, has an inertial manifold when (1) the equation is dissipative, and (2) $f$ is of class $C^{3}$ and for $\Omega_{3}=(0,2 \pi)^{3}$ or $\Omega_{2}=$ $\left(0,2 \pi / a_{1}\right) \times\left(0,2 \pi / a_{2}\right)$, where $a_{1}$ and $a_{2}$ are positive. The proof is based on an (abstract) Invariant Manifold Theorem for flows on a Hilbert space. It is significant that on $\Omega_{3}$ the spectrum of the Laplacian $\Delta$ does not have arbitrary large gaps, as required in other theories of inertial manifolds. Our proof is based on a crucial property of the Schroedinger operator $\Delta+v(x)$, which is valid only in space dimension $n \leq 3$. This property says that $\Delta+v(x)$ can be well approximated by the constant coefficient problem $\Delta+\bar{v}$ over large segments of the Hilbert space $L^{2}(\Omega)$, where $\bar{v}=(\operatorname{vol} \Omega)^{-1} \int_{\Omega} v d x$ is the average value of $v$. We call this property the Principle of Spatial Averaging. The proof that the Schroedinger operator satisfies the Principle of Spatial Averaging on the regions $\Omega_{2}$ and $\Omega_{3}$ described above follows from a gap theorem for finite families of quadratic forms, which we present in an Appendix to this paper.

Division of Applied Mathematics, Brown University, Providence, Rhode Island 02912

Institute for Mathematics and its ApPlications, University of MinNesota, MinNeapoLis, MinNeSOTA 55455 